\title{
Invertibility of adjacency matrices for random $d$-regular graphs
}

\author{
Jiaoyang Huang \\ Harvard University \\ E-mail: jiaoyang@math.harvard.edu
}

\begin{abstract}
Let $d \geqslant 3$ be a fixed integer and $A$ be the adjacency matrix of a random $d$-regular directed or undirected graph on $n$ vertices. We show there exist constants $\mathfrak{d}>0$,

$$
\mathbb{P}(A \text { is singular in } \mathbb{R}) \leqslant n^{-\mathfrak{d}},
$$

for $n$ sufficiently large. This answers an open problem by Frieze [12] and Vu [28,29]. The key idea is to study the singularity probability of adjacency matrices over a finite field $\mathbb{F}_{p}$. The proof combines a local central limit theorem and a large deviation estimate.
\end{abstract}

\section{Introduction}

The most famous combinatorial problem concerning random matrices is perhaps the "singularity" problem. In a standard setting, when the entries of the $n \times n$ matrix are i.i.d. Bernoulli random variables (taking values \pm 1 with probability $1 / 2$ ), this problem was first done by Komlós $[14,15]$, where he showed the probability of being singular is $\mathrm{O}\left(n^{-1 / 2}\right)$. This bound was significantly improved by Kahn, Komlós and Szemerédi [13] to an exponential bound

$$
\mathbb{P}(\text { random Bernoulli matrix is singular })<c^{n},
$$

for $c=0.999$, for $c=3 / 4+\mathrm{o}(1)$ by Tao and $\mathrm{Vu}$ [26], and by Rudelson and Vershynin [24] . The often conjectured optimal value of $c$ is $1 / 2+o(1)$, and the best known value $c=1 / \sqrt{2}+o(1)$ is due to Bourgain, $\mathrm{Vu}$ and Wood [6]. Analogous results on singularity of symmetric Bernoulli matrices were obtained in [9, 22, 27].

The above question can be reformulated for the adjacency matrices of random graphs, either directed or undirected. Both directed and undirected graphs are abundant in real life. One of the widely studied 
model in the undirected random graph literature is the Erdős-Rényi graph $G(n, p)$. It was shown by Costello and $\mathrm{Vu}$ in [10], that the adjacency matrix of $G(n, p)$ is nonsingular with high probability whenever the edge connectivity probability $p$ is above the connectivity threshold $\ln n / n$. For directed Erdős-Rényi graph, a quantitative estimate on the smallest singular value was obtained by Basak and Rudelson in $[2,3]$.

Another intensively studied random graph model is the random $d$-regular graph. For the adjacency matrix of random $d$-regular graphs, its entries are no longer independent. The lack of independence poses significant difficulty for the singularity problem of random $d$-regular graphs. For undirected random $d$ regular graphs, when $d \geqslant n^{c}$ with any $c>0$, it follows from the bulk universality result [16] by Landon, Sosoe and Yau, the adjacency matrix is nonsingular with high probability. For random $d$-regular directed graphs, it was first proven by Cook in [8], the adjacency matrix is nonsingular with high probability when $C \ln ^{2} n \leqslant d \leqslant n-C \ln ^{2} n$. Later in [17], it was proven by Litvak, Lytova, Tikhomirov, Tomczak-Jaegermann and Youssef that, when $C \leqslant d \leqslant n /\left(C \ln ^{2} n\right)$, the singularity probability is bounded by $\mathrm{O}\left(\ln ^{3} d / \sqrt{d}\right)$. Quantitative estimates on the smallest singular values were derived in $[1,7,18]$.

For random $d$-regular graphs, the most challenging case is when $d$ is a constant. It was posted as an open problem first appeared in [28, Conjecture 8.4] by Vu, and later collected in [12, Section 9, Problem 7] by Frieze and [29, Conjecture 5.8] by Vu. In [19], it was proven by Litvak, Lytova, Tikhomirov, TomczakJaegermann and Youssef that the adjacency matrix of random $d$-regular directed graphs has rank at least $n-1$ with high probability. In this paper we prove that the adjacency matrix of random $d$-regular directed and undirected graphs is nonsingular with high probability.

One approach to estimate the singularity probability of random matrices is to decompose the null vectors $\mathbb{S}^{n-1}$ into subsets according to different structural properties, e.g., combinatorial dimension [13, 26], compressible and imcompressible vectors [1,7,8,24,25], and statistics of jumps [2,3,17, 18,20]. Different from previous works, which directly study the singularity probability over $\mathbb{R}$, the key new idea in this paper is to study the singularity probability of adjacency matrices over a finite field $\mathbb{F}_{p}$. At first glance, this may seem wasteful, as we discard a great amount of information. Moreover, as a matrix over $\mathbb{F}_{p}$, the determinant of the adjacency matrix takes value in $\mathbb{F}_{p}$. One expects that the determinant takes value zero with probability about $1 / p$. In other words, the adjacency matrix over $\mathbb{F}_{p}$ may be singular with positive probability. However, the benefit is that, over finite field $\mathbb{F}_{p}$ we can better understand the arithmetic structure of the null vectors, which enables us to obtain a sharp estimate of the singularity probability. We decompose the null vectors $\mathbb{F}_{p}^{n}$ into two classes, the equidistributed class where each number has approximately the same density, and the non-equidistributed class. We estimate the number of adjacency matrices which have a null vector in the equidistributed class using a local central limit theorem, and the number of adjacency matrices which have a null vector in the non-equidistributed class using a large deviation estimate. In [11], Ferber, Luh, McKinley and Samotij use a similar idea to prove resilience results for random Bernoulli matrices.

After the appearance of the current preprint, the asymptotic nonsingularity of adjacency matrices of random $d$-regular directed graphs and random $d$-regular undirected graphs with even number of vertices are proven by Mészáros [21], and by Nguyen and Wood [23]. The work of Mészáros [21] studies the distribution of the sandpile group of random $d$-regular graphs, and determines the distribution of $p$-Sylow subgroup of the sandpile group. Based on [21], Nguyen and Wood in [23], study the distribution of the cokernels of adjacency matrices of random $d$-regular graphs, and observe that the convergence of such distributions implies asymptotic nonsingularity of the matrices. Our model is slightly more general, and we obtain quantitative estimates on the singularity probability.

Acknowledgement. I am thankful to Elchanan Mossel and Mustazee Rahman for suggesting the problem 
of studying adjacency matrices of random $d$-regular graphs over finite fields. I am also grateful to Weifeng Sun for enlightening discussions, and to Nicholas Cook, Van Vu and Melanie Wood for helpful comments on the first draft of this paper.

\subsection{Main results}

We study the configuration model of random $d$-regular directed and undirected graphs, introduced by Bollobás in [5] (ideas similar to the configuration model were also presented in [4,30,31]). By a contiguity argument, our main results also hold for other random $d$-regular directed and undirected graph models, e.g. the uniform model, the sum of $d$ random permutations and the sum of $d$ random perfect matching matrices.

For the configuration model, one generates a random $d$-regular directed graph by the following procedure:

1. Associate to each vertex $k \in\{1,2, \cdots, n\}$ a fiber $F_{k}$ of $d$ points, so that there are $\left|\cup_{k \in\{1,2, \cdots, n\}} F_{k}\right|=n d$ points in total.

2. Select a permutation $\mathcal{P}$ of the $n d$ points uniformly at random.

3. For any vertex $k \in\{1,2, \cdots, n\}$, and point $k^{\prime} \in F_{k}$, we add a directed edge from vertex $k$ to vertex $\ell$ if the point $\mathcal{P}\left(k^{\prime}\right)$ belongs to fiber $F_{\ell}$.

We denote the $d$-regular directed graphs obtained from the above procedure by $\mathrm{M}_{n, d}$, which is a multiset. It is easy to see from the construction procedure that $\left|\mathrm{M}_{n, d}\right|=(n d)$ !. Let $\mathcal{G} \in \mathrm{M}_{n, d}$, one may identify $\mathcal{G}$ with a random $d$-regular bipartite graph on $n+n$ vertices in the obvious way. We denote $A=A(\mathcal{G})$ the adjacency matrix of $\mathcal{G}$, i.e. $A_{k \ell}$ is the number of directed edges from vertex $k$ to vertex $\ell$.

For the configuration model, one generates a random $d$-regular undirected graph on $n$ vertices with $2 \mid d n$, by the following procedure:

1. Associate to each vertex $k \in\{1,2, \cdots, n\}$ a fiber $F_{k}$ of $d$ points, so that there are $\left|\cup_{k \in\{1,2, \cdots, n\}} F_{k}\right|=n d$ points in total.

2. Select a pairing $\mathcal{P}$ of the $n d$ points uniformly at random, and add an edge from point $k^{\prime}$ to point $\ell^{\prime}$ if $\left\{k^{\prime}, \ell^{\prime}\right\} \in \mathcal{P}$.

3. Collapse each fiber $F_{k}$ to the associated vertex $k$.

We denote the $d$-regular undirected graphs obtained from the above procedure by $\mathrm{G}_{n, d}$, which is a multiset. It is easy to see from the construction procedure that $\left|\mathrm{G}_{n, d}\right|=2^{-n d / 2}(n d) ! /(n d / 2)$ !. Let $\mathcal{G} \in \mathrm{G}_{n, d}$, we denote $A=A(\mathcal{G})$ the adjacency matrix of $\mathcal{G}$, i.e. $A_{k \ell}$ is the number of edges between vertex $k$ and vertex $\ell$.

Theorem 1.1. Let $d \geqslant 3$ be a fixed integer, and a prime number $p$ such that $\operatorname{gcd}(p, d)=1$. Then over $\mathbb{F}_{p}$, we have for $p \ll n^{\min \{1 / 4,(d-2) / 2 d\}}$

$$
\sum_{\boldsymbol{v} \in \mathbb{F}_{p}^{n} \backslash \mathbf{0}}\left|\left\{\mathcal{G} \in \mathrm{M}_{n, d}: A(\mathcal{G}) \boldsymbol{v}=\mathbf{0}\right\}\right|=(1+\mathrm{o}(1))\left|\mathrm{M}_{n, d}\right|,
$$


and for $p \ll n^{\min \{1 / 8,(d-2) /(5 d-6)\}}$

$$
\sum_{\boldsymbol{v} \in \mathbb{F}_{p}^{n} \backslash \mathbf{0}}\left|\left\{\mathcal{G} \in \mathrm{G}_{n, d}: A(\mathcal{G}) \boldsymbol{v}=\mathbf{0}\right\}\right|=(1+\mathbf{1}(p=2)+\mathrm{o}(1))\left|\mathrm{G}_{n, d}\right|,
$$

for $n$ sufficiently large.

If an adjacency matrix $A(\mathcal{G})$ is singular as a matrix in $\mathbb{F}_{p}$, then we have

$$
\left|\left\{\boldsymbol{v} \in \mathbb{F}_{p}^{n} \backslash \mathbf{0}: A(\mathcal{G}) \boldsymbol{v}=\mathbf{0}\right\}\right| \geqslant p-1 .
$$

Therefore it follows from Theorem 1.1,

$$
\begin{aligned}
& (p-1) \mid\left\{\mathcal{G} \in \mathrm{M}_{n, d}: A(\mathcal{G}) \text { is singular in } \mathbb{F}_{p}\right\}\left|\leqslant \sum_{\boldsymbol{v} \in \mathbb{F}_{p}^{n} \backslash \mathbf{0}}\right|\left\{\mathcal{G} \in \mathrm{M}_{n, d}: A(\mathcal{G}) \boldsymbol{v}=\mathbf{0}\right\}|=(1+\mathrm{o}(1))| \mathrm{M}_{n, d} \mid, \\
& (p-1) \mid\left\{\mathcal{G} \in \mathrm{G}_{n, d}: A(\mathcal{G}) \text { is singular in } \mathbb{F}_{p}\right\}\left|\leqslant \sum_{\boldsymbol{v} \in \mathbb{F}_{p}^{n} \backslash \mathbf{0}}\right|\left\{\mathcal{G} \in \mathrm{G}_{n, d}: A(\mathcal{G}) \boldsymbol{v}=\mathbf{0}\right\}|=(1+\mathrm{o}(1))| \mathrm{G}_{n, d} \mid,
\end{aligned}
$$

and we obtain the next theorem.

Theorem 1.2. Let $d \geqslant 3$ be a fixed integer, and an odd prime number $p$ such that $\operatorname{gcd}(p, d)=1$. Let $A$ be the adjacency matrix of a random d-regular graph on $n$ vertices. Then as a random matrix in $\mathbb{F}_{p}$,

$$
\mathbb{P}\left(A \text { is singular in } \mathbb{F}_{p}\right) \leqslant \frac{1+\mathrm{o}(1)}{p-1},
$$

for $n$ sufficiently large.

The entries of $A(\mathcal{G})$ are all integers. Therefore, if $A(\mathcal{G})$ is singular in $\mathbb{R}$, it is also singular in any finite field $\mathbb{F}_{p}$. The next theorem follows by taking $p$ large in Theorem 1.2.

Theorem 1.3. Let $d \geqslant 3$ be a fixed integer. Let $A$ be the adjacency matrix of a random d-regular directed or undirected graph on $n$ vertices. Then there exist constants $\mathfrak{d}>0$,

$$
\mathbb{P}(A \text { is singular in } \mathbb{R}) \leqslant n^{-\mathfrak{d}},
$$

for $n$ sufficiently large.

Remark 1.4. For random d-regular directed graphs we can take $\mathfrak{d}=\min \{1 / 4,(d-2) / 2 d\}$, and for random $d$-regular undirected graphs we can take $\mathfrak{d}=\min \{1 / 8,(d-2) /(5 d-6)\}$. The probability that the adjacency matrix of a random $d$-regular graph is singular is at least polynomial in $1 / n$. In fact, if a d-regular graph contains the subgraph in Figure 1, then its adjacency matrix is singular. As a consequence, it holds that $\mathbb{P}(A$ is singular in $\mathbb{R}) \geqslant \mathrm{O}(1) / n^{d-2}$.

Remark 1.5. Theorem 1.3 for random d-regular graphs with even number of vertices was recently proven by Mészáros [21], and by Nguyen and Wood [23]. Their approach studies the distribution of the sandpile group of random d-regular graphs. Although the result in Theorem 1.3 is stated for the configuration model, the same statement holds for other models, e.g. the uniform model, the sum of d random permutations and the sum of d random perfect matching matrices, by a contiguity argument. 

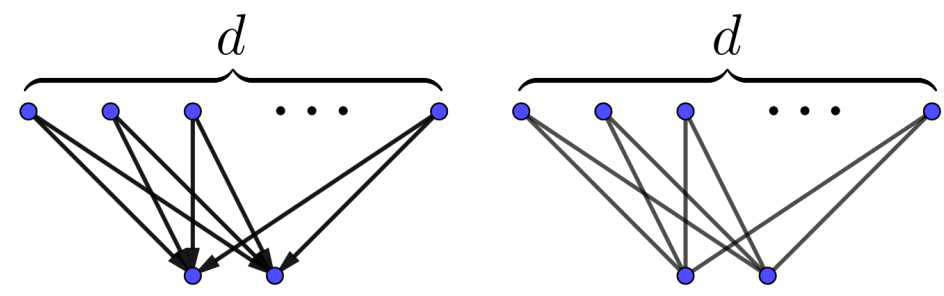

Figure 1: If a $d$-regular directed or undirected graph contains the above subgraph, its adjacency matrix is singular.

\section{Random Walk Interpretation}

In this section, we enumerate $\mid\left\{\mathcal{G} \in \mathrm{M}_{n, d}: A(\mathcal{G}) \boldsymbol{v}=\mathbf{0}\right.$ in $\left.\mathbb{F}_{p}\right\} \mid$ and $\mid\left\{\mathcal{G} \in \mathrm{G}_{n, d}: A(\mathcal{G}) \boldsymbol{v}=\mathbf{0}\right.$ in $\left.\mathbb{F}_{p}\right\} \mid$ as the number of certain walk paths. Before stating the result, we need to introduce some notations. We define the counting function $\Phi: \cup_{k \geqslant 1} \mathbb{F}_{p}^{k} \mapsto \mathbb{Z}^{p}$, given by

$$
\Phi\left(a_{1}, a_{2}, \cdots, a_{k}\right)=\left(\sum_{i=1}^{k} \mathbf{1}\left(a_{i}=0\right), \sum_{i=1}^{k} \mathbf{1}\left(a_{i}=1\right), \cdots, \sum_{i=1}^{k} \mathbf{1}\left(a_{i}=p-1\right)\right) .
$$

We decompose the space $\mathbb{F}_{p}^{n}$ as

$$
\mathbb{F}_{p}^{n}=\bigcup_{\substack{n_{0}, n_{1}, \cdots, n_{p-1} \in \mathbb{Z}_{\geqslant 0} \\ n_{0}+n_{1}+\cdots+n_{p-1}=n}} \mathcal{S}\left(n_{0}, n_{1}, \cdots, n_{p-1}\right)
$$

where

$$
\mathcal{S}\left(n_{0}, n_{1}, \cdots, n_{p-1}\right)=\left\{\boldsymbol{v}=\left(v_{1}, v_{2}, \cdots, v_{n}\right) \in \mathbb{F}_{p}^{n}: \Phi(\boldsymbol{v})=\left(n_{0}, n_{1}, \cdots, n_{p-1}\right)\right\} .
$$

The cardinality of $\mathcal{S}\left(n_{0}, n_{1}, \cdots, n_{p-1}\right)$ is

$$
\left|\mathcal{S}\left(n_{0}, n_{1}, \cdots, n_{p-1}\right)\right|=\left(\begin{array}{c}
n \\
n_{0}, n_{1}, \cdots, n_{p-1}
\end{array}\right) .
$$

We define the multiset $\mathcal{U}_{d, p}$

$$
\mathcal{U}_{d, p}=\left\{\Phi(\boldsymbol{a}): \boldsymbol{a}=\left(a_{1}, a_{2}, \cdots, a_{d}\right) \in \mathbb{F}_{p}^{d}, a_{1}+a_{2}+\cdots+a_{d}=0\right\} .
$$

For any $a_{1}, a_{2}, \cdots, a_{d-1} \in \mathbb{F}_{p}$, there exists a unique $a_{d} \in \mathbb{F}_{p}$ such that $a_{1}+a_{2}+\cdots+a_{d}=0$. The multiset $\mathcal{U}_{d, p}$ has cardinality $p^{d-1}$, i.e. $\left|\mathcal{U}_{d, p}\right|=p^{d-1}$. We denote $\mathcal{M}\left(n_{0}, n_{1}, \cdots, n_{p-1}\right)$ the set of $p \times p$ symmetric matrices $M=\left[m_{i j}\right]_{0 \leqslant i, j \leqslant p-1}$, such that

1. $m_{i j}=m_{j i} \in \mathbb{Z}_{\geqslant 0}$ for $0 \leqslant i, j \leqslant p-1$ and $2 \mid m_{i i}$ for $0 \leqslant i \leqslant p-1$.

2. $\sum_{j=0}^{p-1} m_{i j}=d n_{i}$ for $0 \leqslant i \leqslant p-1$. 
3. $\sum_{j=0}^{p-1} j m_{i j} \equiv 0(\bmod p)$ for $0 \leqslant i \leqslant p-1$.

We denote

$$
\mathcal{M}=\bigcup_{\substack{n_{0}, n_{1}, \cdots, n_{p-1} \in \mathbb{Z}_{\geqslant 0}, n_{0}<n \\ n_{0}+n_{1}+\cdots+n_{p-1}=n}} \mathcal{M}\left(n_{0}, n_{1}, \cdots, n_{p-1}\right) .
$$

Proposition 2.1. Let $d \geqslant 3$ be a fixed integer, and $p$ a prime number. Fix $\boldsymbol{v} \in \mathcal{S}\left(n_{0}, n_{1}, \cdots, n_{p-1}\right)$, we have

$$
\begin{aligned}
& \mid\left\{\mathcal{G} \in \mathrm{M}_{n, d}: A(\mathcal{G}) \boldsymbol{v}=\mathbf{0} \text { in } \mathbb{F}_{p}\right\} \mid \\
= & \left(\prod_{j=0}^{p-1}\left(d n_{j}\right) !\right)\left|\left\{\left(\boldsymbol{u}_{1}, \boldsymbol{u}_{2} \cdots, \boldsymbol{u}_{n}\right) \in \mathcal{U}_{d, p}^{n}: \boldsymbol{u}_{1}+\boldsymbol{u}_{2}+\cdots+\boldsymbol{u}_{n}=\left(d n_{0}, d n_{1}, \cdots, d n_{p-1}\right)\right\}\right| \\
= & \left(\prod_{j=0}^{p-1}\left(d n_{j}\right) !\right) p^{n(d-1)} \mathbb{P}\left(X_{1}+X_{2}+\cdots+X_{n}=\left(d n_{0}, d n_{1}, \cdots, d n_{p-1}\right)\right),
\end{aligned}
$$

where $X_{1}, X_{2}, \cdots, X_{n}$ are independent copies of $X$, which is uniform distributed over $\mathcal{U}_{d, p}$.

Proposition 2.2. Let $d \geqslant 3$ be a fixed integer, and $p$ a prime number. Fix $\boldsymbol{v} \in \mathcal{S}\left(n_{0}, n_{1}, \cdots, n_{p-1}\right)$, we have

$$
\begin{aligned}
& \mid\left\{\mathcal{G} \in \mathrm{G}_{n, d}: A(\mathcal{G}) \boldsymbol{v}=\mathbf{0} \text { in } \mathbb{F}_{p}\right\} \mid \\
= & \sum_{M \in \mathcal{M}\left(n_{0}, n_{1}, \cdots, n_{p-1}\right)} \prod_{0 \leqslant i<j \leqslant p-1} m_{i j} ! \prod_{i=0}^{p-1} \frac{m_{i i} !}{2^{m_{i i} / 2}\left(m_{i i} / 2\right) !} \\
\times & \prod_{i=0}^{p-1}\left|\left\{\left(\boldsymbol{u}_{1}, \boldsymbol{u}_{2} \cdots, \boldsymbol{u}_{n_{i}}\right) \in \mathcal{U}_{d, p}^{n_{i}}: \boldsymbol{u}_{1}+\boldsymbol{u}_{2}+\cdots \boldsymbol{u}_{n_{i}}=\left(m_{i 0}, m_{i 1}, \cdots, m_{i p-1}\right)\right\}\right| \\
= & \sum_{M \in \mathcal{M}\left(n_{0}, n_{1}, \cdots, n_{p-1}\right)} \prod_{0 \leqslant i<j \leqslant p-1} m_{i j} ! \prod_{i=0}^{p-1} \frac{m_{i i} !}{2^{m_{i i} / 2}\left(m_{i i} / 2\right) !} p^{n(d-1)} \\
& \times \prod_{i=0}^{p-1} \mathbb{P}\left(X_{1}+X_{2}+\cdots+X_{n_{i}}=\left(m_{i 0}, m_{i 1}, \cdots, m_{i p-1}\right)\right),
\end{aligned}
$$

where $X_{1}, X_{2}, \cdots, X_{n_{i}}$ are independent copies of $X$, which is uniform distributed over $\mathcal{U}_{d, p}$.

Proof of Proposition 2.1. We recall the configuration model for random $d$-regular directed graphs from the introduction that each vertex $k \in\{1,2, \cdots, n\}$ is associated with a fiber $F_{k}$ of $d$ points. For each permutation $\mathcal{P}$ of the $n d$ points, we associate it a map $f_{\mathcal{P}}: \cup_{k \in\{1,2, \cdots, n\}} F_{k} \mapsto \mathbb{F}_{p}$ in the following way. For any point $k^{\prime}$, if $\mathcal{P}\left(k^{\prime}\right)=\ell^{\prime}$ and $\ell^{\prime} \in F_{\ell}$, then $f_{\mathcal{P}}\left(k^{\prime}\right)=v_{\ell}$. A given map $f: \cup_{k \in\{1,2, \cdots, n\}} F_{k} \mapsto \mathbb{F}_{p}$ is from a permutation if

$$
\sum_{k \in\{1,2, \cdots, n\}} \sum_{k^{\prime} \in F_{k}} \mathbf{1}\left(f\left(k^{\prime}\right)=j\right)=d n_{j}, \quad i=0,1, \cdots, p-1 .
$$

If this is the case, the number of permutation $\mathcal{P}$ such that $f_{\mathcal{P}}=f$ is given by

$$
\prod_{j=0}^{p-1}\left(d n_{j}\right) !
$$


Let $\mathcal{G} \in \mathrm{M}_{n, d}$ corresponding to a permutation $\mathcal{P} . A(\mathcal{G}) \boldsymbol{v}=\mathbf{0}$ in $\mathbb{F}_{p}$ if and only if for any $k \in\{1,2, \cdots, n\}$

$$
\sum_{k^{\prime} \in F_{k}} f\left(k^{\prime}\right)=0 .
$$

Especially, $\Phi\left(f\left(k^{\prime}\right): k^{\prime} \in F_{k}\right) \in \mathcal{U}_{d, p}$ for any $k \in\{1,2, \cdots, n\}$. The number of maps $f: \cup_{k \in\{1,2, \cdots, n\}} F_{k} \mapsto \mathbb{F}_{p}$ satisfying (2.4) and (2.6) is given by

$$
\left|\left\{\left(\boldsymbol{u}_{1}, \boldsymbol{u}_{2} \cdots, \boldsymbol{u}_{n}\right) \in \mathcal{U}_{d, p}^{n}: \boldsymbol{u}_{1}+\boldsymbol{u}_{2}+\cdots+\boldsymbol{u}_{n}=\left(d n_{0}, d n_{1}, \cdots, d n_{p-1}\right)\right\}\right| .
$$

The claim (2.2) follows from (2.5) and (2.7).

Proof of Proposition 2.2. We recall the configuration model for random $d$-regular undirected graphs from the introduction that each vertex $k \in\{1,2, \cdots, n\}$ is associated with a fiber $F_{k}$ of $d$ points. For each pairing $\mathcal{P}$ of the $n d$ points, we associate it a map $f_{\mathcal{P}}: \cup_{k \in\{1,2, \cdots, n\}} F_{k} \mapsto \mathbb{F}_{p}$ in the following way. For any point $k^{\prime}$, if $\left\{k^{\prime}, \ell^{\prime}\right\} \in \mathcal{P}$ and $\ell^{\prime} \in F_{\ell}$, then $f_{\mathcal{P}}\left(k^{\prime}\right)=v_{\ell}$. Given a map $f: \cup_{k \in\{1,2, \cdots, n\}} F_{k} \mapsto \mathbb{F}_{p}$, we denote its data matrix as

$$
M_{f}=\left[m_{i j}\right]_{0 \leqslant i, j \leqslant p-1}, \quad m_{i j}=\sum_{k \in\{1,2, \cdots, n\}} \sum_{k^{\prime} \in F_{k}} \mathbf{1}\left(v_{k}=i\right) \mathbf{1}\left(f\left(k^{\prime}\right)=j\right) .
$$

The map $f: \cup_{k \in\{1,2, \cdots, n\}} F_{k} \mapsto \mathbb{F}_{p}$ is from a pairing $\mathcal{P}$ if for any $\left\{k^{\prime}, \ell^{\prime}\right\} \in \mathcal{P}$ with $k^{\prime} \in F_{k}$ and $\ell^{\prime} \in F_{\ell}$, it holds $f\left(k^{\prime}\right)=v_{\ell}$ and $f\left(\ell^{\prime}\right)=v_{k}$. This is possible, if and only if its data matrix $M_{f}=\left[m_{i j}\right]_{0 \leqslant i, j \leqslant p-1}$ satisfies

1. $m_{i j}=m_{j i} \in \mathbb{Z}_{\geqslant 0}$ for $0 \leqslant i, j \leqslant p-1$ and $2 \mid m_{i i}$ for $0 \leqslant i \leqslant p-1$.

2. $\sum_{j=0}^{p-1} m_{i j}=d n_{i}$ for $0 \leqslant i \leqslant p-1$.

If this is the case, the number of pairings $\mathcal{P}$ such that $f_{\mathcal{P}}=f$ is given by

$$
\prod_{0 \leqslant i<j \leqslant p-1} m_{i j} ! \prod_{i=0}^{p-1} \frac{m_{i i} !}{2^{m_{i i} / 2}\left(m_{i i} / 2\right) !} .
$$

Let $\mathcal{G} \in \mathrm{G}_{n, d}$ corresponding to a pairing $\mathcal{P} . A(\mathcal{G}) \boldsymbol{v}=\mathbf{0}$ in $\mathbb{F}_{p}$ if and only if for any $k \in\{1,2, \cdots, n\}$

$$
\sum_{k^{\prime} \in F_{k}} f\left(k^{\prime}\right)=0
$$

Especially, $\Phi\left(f\left(k^{\prime}\right): k^{\prime} \in F_{k}\right) \in \mathcal{U}_{d, p}$ for any $k \in\{1,2, \cdots, n\}$. If this is the case, we have

$$
0=\sum_{k \in\{1,2, \cdots, n\}} \mathbf{1}\left(v_{k}=i\right) \sum_{k^{\prime} \in F_{k}} f\left(k^{\prime}\right) \equiv \sum_{j=0}^{p-1} j m_{i j}(\bmod p),
$$

and thus the data matrix $M_{f} \in \mathcal{M}\left(n_{0}, n_{1}, \cdots, n_{p-1}\right)$. Given a data matrix $M \in \mathcal{M}\left(n_{0}, n_{1}, \cdots, n_{p-1}\right)$, the number of maps $f: \cup_{k \in\{1,2, \cdots, n\}} F_{k} \mapsto \mathbb{F}_{p}$ with data matrix $M$ satisfying (2.9) is given by

$$
\prod_{i=0}^{p-1}\left|\left\{\left(\boldsymbol{u}_{1}, \boldsymbol{u}_{2} \cdots, \boldsymbol{u}_{n_{i}}\right) \in \mathcal{U}_{d, p}^{n_{i}}: \boldsymbol{u}_{1}+\boldsymbol{u}_{2}+\cdots \boldsymbol{u}_{n_{i}}=\left(m_{i 0}, m_{i 1}, \cdots, m_{i p-1}\right)\right\}\right| .
$$

The claim (2.3) follows from (2.8) and (2.10). 
Let $X$ be a random vector uniform distributed over $\mathcal{U}_{d, p}$. The mean of $X$ is given by

$$
\mathbb{E}[X(j)]=\frac{1}{p^{d-1}} \sum_{\substack{\left(a_{1}, a_{2}, \cdots, a_{d}\right) \in \mathbb{F}_{p}^{d} \\ a_{1}+a_{2}+\cdots+a_{d}=0}} \sum_{k=1}^{d} \mathbf{1}\left(a_{k}=j\right)=\frac{d}{p}, \quad j=0,1, \cdots, p-1 .
$$

The covariance of $X$ is given by

$$
\begin{aligned}
& \mathbb{E}\left[(X(j)-d / p)\left(X\left(j^{\prime}\right)-d / p\right)\right]=\frac{1}{p^{d-1}} \sum_{\substack{\left(a_{1}, a_{2}, \cdots, a_{d}\right) \in \mathbb{F}_{p}^{d} \\
a_{1}+a_{2}+\cdots+a_{d}=0}} \sum_{\substack{1 \leqslant k, k^{\prime} \leqslant d \\
p^{d-1}}} \sum_{\substack{\left(a_{1}, a_{2}, \cdots, a_{d}\right) \in \mathbb{P}_{p}^{d} \\
a_{1}+a_{2}+\cdots+a_{d}=0}}\left(\delta_{j j^{\prime}} \sum_{1 \leqslant k \leqslant d} \mathbf{1}\left(a_{k}=j\right) \mathbf{1}\left(a_{k^{\prime}}=j^{\prime}\right)-\frac{d^{2}}{p^{2}}\right. \\
&\left.=\sum_{1 \leqslant k \neq k^{\prime} \leqslant d} \mathbf{1}\left(a_{k}=j\right) \mathbf{1}\left(a_{k^{\prime}}=j^{\prime}\right)\right)-\frac{d^{2}}{p^{2}}=\frac{d}{p} \delta_{j j^{\prime}}-\frac{d}{p^{2}},
\end{aligned}
$$

for any $j, j^{\prime}=0,1, \cdots, p-1$. We summarize (2.11) and (2.12) as

$$
\boldsymbol{\mu}:=\mathbb{E}[X]=(d / p, d / p, \cdots, d / p), \quad \Sigma:=\mathbb{E}\left[(X-\boldsymbol{\mu})(X-\boldsymbol{\mu})^{t}\right]=\frac{d}{p} I_{p}-\frac{d}{p^{2}} \mathbf{1 1}^{t} .
$$

We denote the characteristic function of $X$ as

$$
\phi_{X}(\boldsymbol{t})=\mathbb{E}[\exp \{\mathrm{i}\langle\boldsymbol{t}, X\rangle\}], \quad \phi_{X-\boldsymbol{\mu}}(\boldsymbol{t})=\mathbb{E}[\exp \{\mathrm{i}\langle\boldsymbol{t}, X-\boldsymbol{\mu}\rangle\}]=e^{-\mathrm{i}\langle\boldsymbol{t}, \boldsymbol{\mu}\rangle} \phi_{X}(\boldsymbol{t}) .
$$

The lattice spanned by vectors in $\mathcal{U}_{d, p}$ is the dual lattice of $\operatorname{span}\left\{(0,1 / p, 2 / p, \cdots, p-1 / p), \boldsymbol{e}_{1}, \boldsymbol{e}_{2}, \cdots, \boldsymbol{e}_{p}\right\}$ in $\left\{\left(x_{1}, x_{2}, \cdots, x_{p}\right) \in \mathbb{R}^{p}: x_{1}+x_{2}+\cdots+x_{p}=d\right\}$, where $\boldsymbol{e}_{1}, \boldsymbol{e}_{2}, \cdots, \boldsymbol{e}_{p}$ is the standard base of $\mathbb{R}^{p}$. Therefore $\left|\phi_{X-\boldsymbol{\mu}}^{n}(\boldsymbol{t})\right|=1$ if any only if

$$
\boldsymbol{t} \in 2 \pi(0,1 / p, 2 / p, \cdots, p-1 / p) \mathbb{Z}+(1,1, \cdots, 1) \mathbb{R} .
$$

The following proposition gives a quantitative estimate of the absolute value of the characteristic function $\left|\phi_{X-\mu}(\boldsymbol{t})\right|$ when $\boldsymbol{t}$ is away from these lines (2.14). Fix small $\delta>0$. For $j=0,1,2, \cdots, p-1$, we define domains

$$
B_{j}(\delta)=2 \pi j(0,1 / p, 2 / p, \cdots,(p-1) / p)+Q\left(\left\{\boldsymbol{x} \in \mathbb{R}^{p-1}:\|\boldsymbol{x}\|_{2}^{2} \leqslant \delta\right\} \times[0,2 \sqrt{p} \pi]\right),
$$

where $Q$ is an orthogonal transform, given by the $p \times p$ orthogonal matrix $Q=[O, \mathbf{1} / \sqrt{p}]$, and $Q\left(\left\{\boldsymbol{x} \in \mathbb{R}^{p-1}\right.\right.$ : $\left.\left.\|\boldsymbol{x}\|_{2}^{2} \leqslant \delta\right\} \times[0,2 \sqrt{p} \pi]\right)$ is the image of $\left\{\boldsymbol{x} \in \mathbb{R}^{p-1}:\|\boldsymbol{x}\|_{2}^{2} \leqslant \delta\right\} \times[0,2 \sqrt{p} \pi]$ under the orthogonal transform $Q$.

Proposition 2.3. For any $\delta>0$ small enough, and $\boldsymbol{t} \in\left(2 \pi \mathbb{R}^{p} / \mathbb{Z}^{p}\right) \backslash \cup_{j=0}^{p-1} B_{j}(\delta)$, there exists a constant $c(\delta)>0$,

$$
\left|\phi_{X-\mu}(\boldsymbol{t})\right| \leqslant 1-c(\delta) / p^{3} .
$$

Proof. We prove (2.16) by contradiction: if for some $\boldsymbol{t}$,

$$
\left|\frac{1}{p^{d-1}} \sum_{j=1}^{p^{d-1}} e^{\mathrm{i}\left\langle\boldsymbol{t}, \boldsymbol{w}_{j}\right\rangle}\right| \geqslant 1-c(\delta) / p^{3},
$$


then $\boldsymbol{t} \in \cup_{j=0}^{p-1} B_{j}(\delta)$. Without loss of generality, we can assume (by shifting $\boldsymbol{t}$ ) that $t_{0}=0$ and $\left|t_{1}\right| \leqslant 1 / 2 p$. We denote $\psi=\arg \phi_{X}(\boldsymbol{t})$. There exists a subset $\mathcal{U}^{\prime} \in \mathcal{U}_{d, p}$, with $\left|\mathcal{U}^{\prime}\right| \leqslant p^{d-2} /(2 d)$, such that for any $\boldsymbol{w} \in \mathcal{U}_{d, p} \backslash \mathcal{U}^{\prime}$, it holds

$$
\langle\boldsymbol{t}, \boldsymbol{w}\rangle=2 \pi n_{\boldsymbol{w}}+\psi+\varepsilon_{\boldsymbol{w}}, \quad\left|\varepsilon_{\boldsymbol{w}}\right| \leqslant \frac{\varepsilon}{p},
$$

where $1-\cos (\varepsilon / p)=2 d c(\delta) / p^{2}$. Otherwise, we have

$$
\begin{aligned}
\left|\frac{1}{p^{d-1}} \sum_{j=1}^{p^{d-1}} e^{\mathrm{i}\left\langle\boldsymbol{t}, \boldsymbol{w}_{j}\right\rangle}\right| & =\operatorname{Re} \frac{1}{p^{d-1}} \sum_{j=1}^{p^{d-1}} e^{\mathrm{i}\left\langle\boldsymbol{t}, \boldsymbol{w}_{j}\right\rangle-\mathrm{i} \psi} \\
& <\frac{1}{p^{d-1}}\left(\left|\mathcal{U}_{d, p} \backslash \mathcal{U}^{\prime}\right|+\sum_{\boldsymbol{w} \in \mathcal{U}^{\prime}} \cos (\varepsilon / p)\right)=1-\frac{c(\delta)}{p^{3}},
\end{aligned}
$$

which contradicts with (2.17). We show next that (2.18) holds for all $\boldsymbol{w} \in \mathcal{U}_{d, p}$ with slightly worse error. We consider $d \times d$ arrays in $\mathbb{F}_{p}$ such that the sum of each row and column is zero (zero sum $d \times d$ arrays). Fix any $\boldsymbol{a}^{1}=\left(a_{1}^{1}, a_{2}^{1}, \cdots, a_{d}^{1}\right) \in \mathbb{F}_{p}^{d}$ with $a_{1}^{1}+a_{2}^{1}+\cdots+a_{d}^{1}=0$. The total number of zero sum $d \times d$ arrays with the first row given by $\boldsymbol{a}^{1}$ is $p^{(d-1)(d-2)}$. For any $\boldsymbol{b}=\left(b_{1}, b_{2}, \cdots, b_{d}\right) \in \mathbb{F}_{p}^{d}$ with $b_{1}+b_{2}+\cdots+b_{d}=0$ and $\boldsymbol{b} \neq \boldsymbol{a}^{1}$, the total number of zero sum $d \times d$ arrays with the first row given by $\boldsymbol{a}^{1}$ and one row or column given by $\boldsymbol{b}$ is at most $(d-1) p^{(d-1)(d-3)}+d p^{(d-2)(d-2)}$. Since $p^{(d-1)(d-2)}>\left((d-1) p^{(d-1)(d-3)}+d p^{(d-2)(d-2)}\right)\left|\mathcal{U}^{\prime}\right|$, there exists a zero sum $d \times d$ arrays with rows given by $\boldsymbol{a}^{1}, \boldsymbol{a}^{2}, \cdots, \boldsymbol{a}^{d}$ and columns given by $\boldsymbol{b}^{1}, \boldsymbol{b}^{2}, \cdots, \boldsymbol{b}^{d}$ such that $\Phi\left(\boldsymbol{a}^{2}\right), \Phi\left(\boldsymbol{a}^{3}\right), \cdots, \Phi\left(\boldsymbol{a}^{d}\right), \Phi\left(\boldsymbol{b}^{1}\right), \Phi\left(\boldsymbol{b}^{2}\right), \cdots, \Phi\left(\boldsymbol{b}^{d}\right) \in \mathcal{U}_{b, p} \backslash \mathcal{U}^{\prime}$. As a consequence, we have

$$
\begin{aligned}
\left\langle\boldsymbol{t}, \Phi\left(\boldsymbol{a}^{1}\right)\right\rangle & =\sum_{i=1}^{d}\left\langle\boldsymbol{t}, \Phi\left(\boldsymbol{b}^{i}\right)\right\rangle-\sum_{i=2}^{d}\left\langle\boldsymbol{t}, \Phi\left(\boldsymbol{a}^{i}\right)\right\rangle \\
& =2 \pi\left(\sum_{i=1}^{d} n_{\Phi\left(\boldsymbol{b}^{i}\right)}-\sum_{i=2}^{d} n_{\Phi\left(\boldsymbol{a}^{i}\right)}\right)+\psi+\left(\sum_{i=1}^{d} \varepsilon_{\Phi\left(\boldsymbol{b}^{i}\right)}-\sum_{i=2}^{d} \varepsilon_{\Phi\left(\boldsymbol{a}^{i}\right)}\right) \\
& =: 2 \pi n_{\Phi\left(\boldsymbol{a}^{1}\right)}+\psi+\varepsilon_{\Phi\left(\boldsymbol{a}^{1}\right)},
\end{aligned}
$$

where $\left|\varepsilon_{\Phi\left(\boldsymbol{a}^{1}\right)}\right| \leqslant 2 d \varepsilon / p$. Especially if we take $\boldsymbol{a}^{1}=(0,0, \cdots, 0)$ then $\left\langle\boldsymbol{t}, \Phi\left(\boldsymbol{a}^{1}\right)\right\rangle=0$, and we get $|\psi| \leqslant 2 d \varepsilon / p$. We can absorb $\psi$ into the error term. Therefore, uniformly for all $\boldsymbol{w} \in \mathcal{U}_{d, p}$,

$$
\langle\boldsymbol{t}, \boldsymbol{w}\rangle=2 \pi n_{\boldsymbol{w}}+\varepsilon_{\boldsymbol{w}}, \quad\left|\varepsilon_{\boldsymbol{w}}\right| \leqslant \frac{4 d \varepsilon}{p} .
$$

We take two family of vectors $\boldsymbol{u}_{k}=(d-2) \boldsymbol{e}_{0}+\boldsymbol{e}_{k}+\boldsymbol{e}_{p-k}, \boldsymbol{v}_{k}=(d-3) \boldsymbol{e}_{0}+\boldsymbol{e}_{1}+\boldsymbol{e}_{k-1}+\boldsymbol{e}_{p-k} \in \mathcal{U}_{d, p}$ for $k=2,3, \cdots, p-1$. By taking $\boldsymbol{w}=\boldsymbol{u}_{k}, \boldsymbol{v}_{k}$ in (2.19), we get

$$
t_{k}=t_{k-1}+t_{1}+2 \pi\left(n_{\boldsymbol{v}_{k}}-n_{\boldsymbol{u}_{k}}\right)+\left(\varepsilon_{\boldsymbol{v}_{k}}-\varepsilon_{\boldsymbol{u}_{k}}\right), \quad k=2,3, \cdots, p-1,
$$

and therefore,

$$
t_{k}=k t_{1}+2 \pi \sum_{j=2}^{k}\left(n_{\boldsymbol{v}_{j}}-n_{\boldsymbol{u}_{j}}\right)+\sum_{j=2}^{k}\left(\varepsilon_{\boldsymbol{v}_{j}}-\varepsilon_{\boldsymbol{u}_{j}}\right) .
$$


We can shift each $t_{k}$ by $2 \pi \mathbb{Z}$, and assume that

$$
t_{k}=k t_{1}+\sum_{j=2}^{k}\left(\varepsilon_{\boldsymbol{v}_{j}}-\varepsilon_{\boldsymbol{u}_{j}}\right) .
$$

Therefore, thanks to the bound (2.19), we get $\left|t_{k}\right| \leqslant k / 2 p+8(k-1) d \varepsilon / p \leqslant 1$ for all $1 \leqslant k \leqslant p-1$, provided we take $\varepsilon$ small enough. Let $k_{\max }=\operatorname{argmax}_{k} t_{k}$ and $k_{\min }=\operatorname{argmin}_{k} t_{k}$. By taking $\boldsymbol{w}_{1}=(d-2) \boldsymbol{e}_{0}+\boldsymbol{e}_{k_{\max }}+$ $\boldsymbol{e}_{p-k_{\max }}$ and $\boldsymbol{w}_{2}=(d-3) \boldsymbol{e}_{0}+\boldsymbol{e}_{k_{\min }}+\boldsymbol{e}_{k_{\max }-k_{\min }}+\boldsymbol{e}_{p-k_{\max }}$ in (2.19), we get

$$
3 \geqslant\left|t_{k_{\max }}-t_{k_{\min }}-t_{k_{\max }-k_{\min }}\right|=\left|2 \pi\left(n_{\boldsymbol{w}_{2}}-n_{\boldsymbol{w}_{1}}\right)+\left(\varepsilon_{\boldsymbol{w}_{2}}-\varepsilon_{\boldsymbol{w}_{1}}\right)\right| \geqslant 2 \pi\left|n_{\boldsymbol{w}_{2}}-n_{\boldsymbol{w}_{1}}\right|-\left|\varepsilon_{\boldsymbol{w}_{2}}-\varepsilon_{\boldsymbol{w}_{1}}\right| .
$$

Therefore, $n_{\boldsymbol{w}_{2}}=n_{\boldsymbol{w}_{1}}$ and

$$
\left|t_{k_{\min }}\right| \leqslant\left|t_{k_{\max }}-t_{k_{\min }}-t_{k_{\max }-k_{\min }}\right|=\left|\varepsilon_{\boldsymbol{w}_{2}}-\varepsilon_{\boldsymbol{w}_{1}}\right| \leqslant \frac{8 d \varepsilon}{p} .
$$

By symmetry, we also have $\left|t_{k_{\max }}\right| \leqslant 8 d \varepsilon / p$. It follows that $\left|t_{0}\right|,\left|t_{1}\right|, \cdots,\left|t_{p-1}\right| \leqslant \max \left\{\left|t_{k_{\max }}\right|,\left|t_{k_{\min }}\right|\right\} \leqslant$ $8 d \varepsilon / p$. So $\boldsymbol{t} \in B_{0}(\delta)$ and the claim follows, provided we take $\varepsilon$ small enough.

\section{Proof of Theorem 1.1 for Random Directed $d$-regular Graphs}

Thanks to Proposition 2.1, we can rewrite the lefthand side of (1.1) as

$$
\begin{aligned}
\sum_{\boldsymbol{v} \in \mathbb{F}_{p}^{n} \backslash \mathbf{0}}\left|\left\{\mathcal{G} \in \mathrm{M}_{n, d}: A(\mathcal{G}) \boldsymbol{v}=\mathbf{0}\right\}\right|= & \sum_{\substack{n_{0}, n_{1}, \cdots, n_{p-1} \in \mathbb{Z}_{\geqslant 0}, n_{0}<n \\
n_{0}+n_{1}+\cdots+n_{p-1}=n}} \sum_{\boldsymbol{v} \in \mathcal{S}\left(n_{0}, n_{1}, \cdots, n_{p-1}\right)}\left|\left\{\mathcal{G} \in \mathrm{M}_{n, d}: A(\mathcal{G}) \boldsymbol{v}=\mathbf{0}\right\}\right| \\
= & \sum_{\substack{n_{0}, n_{1}, \cdots, n_{p-1} \in \mathbb{Z}_{\geqslant 0}, n_{0}<n \\
n_{0}+n_{1}+\cdots+n_{p-1}=n}}\left(\begin{array}{c}
n \\
n_{0}, n_{1}, \cdots, n_{p-1}
\end{array}\right)\left(\prod_{j=0}^{p-1}\left(d n_{j}\right) !\right) \times \\
& \times p^{n(d-1)} \mathbb{P}\left(X_{1}+X_{2}+\cdots+X_{n}=\left(d n_{0}, d n_{1}, \cdots, d n_{p-1}\right)\right) .
\end{aligned}
$$

Therefore Theorem 1.1 is equivalent to the following estiamte

$$
\begin{gathered}
\sum_{\substack{n_{0}, n_{1}, \cdots, n_{p-1} \in \mathbb{Z}_{\geqslant 0}, n_{0}<n \\
n_{0}+n_{1}+\cdots+n_{p-1}=n}}\left(\begin{array}{c}
n \\
n_{0}, n_{1}, \cdots, n_{p-1}
\end{array}\right)\left(\begin{array}{c}
d n \\
d n_{0}, d n_{1}, \cdots, d n_{p-1}
\end{array}\right)^{-1} \times \\
\times p^{n(d-1)} \mathbb{P}\left(X_{1}+X_{2}+\cdots+X_{n}=\left(d n_{0}, d n_{1}, \cdots, d n_{p-1}\right)\right)=1+\mathrm{o}(1) .
\end{gathered}
$$

To prove (3.1), we fix a large number $\mathfrak{b}>0$, and decompose those $p$-tuples $\left(n_{0}, n_{1}, \cdots, n_{p-1}\right)$ into two classes:

1. (Equidistributed) $\mathcal{E}$ is the set of $p$-tuples $\left(n_{0}, n_{1}, \cdots, n_{p-1}\right) \in \mathbb{Z}_{\geqslant 0}^{n}$, such that $\sum_{j=0}^{p-1}\left(n_{j} / n-1 / p\right)^{2} \leqslant$ $\mathfrak{b} \ln n / n$.

2. (Non-equidistributed) $\mathcal{N}$ is the set of $p$-tuples $\left(n_{0}, n_{1}, \cdots, n_{p-1}\right) \in \mathbb{Z}_{\geqslant 0}^{n}$, which are not $(n, 0,0, \cdots, 0)$ or equidistributed. 
In Section 3.1, we estimate the sum of terms in (3.1) corresponding to equidistributed $p$-tuples using a local central limit theorem. In Section 3.2, we show that the sum of terms in (3.1) corresponding to nonequidistributed $p$-tuples is small, via a large deviation estimate. Theorem 1.1 for random directed $d$-regular graphs follows from combining Proposition 3.1 and Proposition 3.2.

\subsection{Local central limit theorem estimate}

In this section, we estimate the sum of terms in (3.1) corresponding to equidistributed $p$-tuples $\left(n_{0}, n_{1}, \cdots, n_{p-1}\right)$, using a local central limit theorem.

Proposition 3.1. Let $d \geqslant 3$ be a fixed integer, and a prime number $p$ such that $\operatorname{gcd}(p, d)=1$ and $p \ll n^{1 / 4}$. Then for $n$ sufficiently large

$$
\sum_{\left(n_{0}, n_{1}, \cdots, n_{p-1}\right) \in \mathcal{E} \boldsymbol{v} \in \mathcal{S}\left(n_{0}, n_{1}, \cdots, n_{p-1}\right)}\left|\left\{\mathcal{G} \in \mathrm{M}_{n, d}: A(\mathcal{G}) \boldsymbol{v}=\mathbf{0}\right\}\right|=\left(1+\mathrm{O}\left(\frac{p^{2}(\ln n)^{3 / 2}}{\sqrt{n}}\right)\right)\left|\mathrm{M}_{n, d}\right|
$$

Proof. Thanks to Proposition 2.1, we have

$$
\begin{aligned}
& \frac{1}{\left|\mathrm{M}_{n, d}\right|} \sum_{\left(n_{0}, n_{1}, \cdots, n_{p-1}\right) \in \mathcal{E}} \sum_{\boldsymbol{v} \in \mathcal{S}\left(n_{0}, n_{1}, \cdots, n_{p-1}\right)}\left|\left\{\mathcal{G} \in \mathrm{M}_{n, d}: A(\mathcal{G}) \boldsymbol{v}=\mathbf{0}\right\}\right| \\
& =\sum_{\left(n_{0}, n_{1}, \cdots, n_{p-1}\right) \in \mathcal{E}}\left(\begin{array}{c}
n \\
n_{0}, n_{1}, \cdots, n_{p-1}
\end{array}\right)\left(\begin{array}{c}
d n \\
d n_{0}, d n_{1}, \cdots, d n_{p-1}
\end{array}\right)^{-1} \\
& \times p^{n(d-1)} \mathbb{P}\left(X_{1}+X_{2}+\cdots+X_{n}=\left(d n_{0}, d n_{1}, \cdots, d n_{p-1}\right)\right),
\end{aligned}
$$

where $X_{1}, X_{2}, \cdots, X_{n}$ are independent copies of $X$, which is uniform distributed over $\mathcal{U}_{d, p}$ as defined in (2.1). For an equidistributed $p$-tuple $\left(n_{0}, n_{1}, \cdots, n_{p-1}\right)$, we denote $\mathfrak{n}_{j}=n_{j} / n$ for $j=0,1, \cdots, p-1$. Then by our definition of $\mathcal{E}$, we have $\sum_{j=0}^{p-1}\left(\mathfrak{n}_{j}-1 / p\right)^{2} \leqslant \mathfrak{b} \ln n / n$. We estimate the first factor on the righthand side of (3.3) using Stirling's formula,

$$
\begin{aligned}
& \left(\begin{array}{c}
n \\
n_{0}, n_{1}, \cdots, n_{p-1}
\end{array}\right)\left(\begin{array}{c}
d n \\
d n_{0}, d n_{1}, \cdots, d n_{p-1}
\end{array}\right)^{-1} p^{(d-1) n} \\
= & \left(1+\mathrm{O}\left(\frac{p^{2}}{n}\right)\right) d^{\frac{p-1}{2}} \exp \left\{(d-1) n\left(\sum_{j=0}^{p-1} \mathfrak{n}_{j} \ln \mathfrak{n}_{j}+\ln p\right)\right\} \\
= & \left(1+\mathrm{O}\left(\frac{p(\ln n)^{3 / 2}}{\sqrt{n}}\right)\right) d^{\frac{p-1}{2}} \exp \left\{\frac{(d-1) p n}{2} \sum_{j=0}^{p-1}\left(\mathfrak{n}_{j}-1 / p\right)^{2}\right\} .
\end{aligned}
$$

In the following, we estimate $\mathbb{P}\left(S_{n}=\left(d n_{0}, d n_{1}, \cdots, d n_{p-1}\right)\right)$, where $S_{n}=X_{1}+X_{2}+\cdots+X_{n}$. We recall that $X_{1}, X_{2}, \cdots, X_{n}$ are independent copies of $X$, which is uniformly distributed over the multiset $\mathcal{U}_{d, p}$. For a $p$-tuple $\left(n_{0}, n_{1}, \cdots, n_{p-1}\right)$, if $\sum_{j=0}^{p-1} j n_{j} \not \equiv 0(\bmod p)$, then $\mathbb{P}\left(S_{n}=\left(d n_{0}, d n_{1}, \cdots, d n_{p-1}\right)\right)=0$. We only need to consider $p$-tuples $\left(n_{0}, n_{1}, \cdots, n_{p-1}\right)$ such that $\sum_{j=0}^{p-1} j n_{j} \equiv 0(\bmod p)$. We denote $\boldsymbol{n}=\left(n_{0}, n_{1}, \cdots, n_{p-1}\right)$. 
By inverse Fourier formula

$$
\begin{aligned}
\mathbb{P}\left(S_{n}=d \boldsymbol{n}\right) & =\frac{1}{(2 \pi)^{p}} \int_{2 \pi \mathbb{R}^{p} / \mathbb{Z}^{p}} \phi_{X}^{n}(\boldsymbol{t}) e^{-\mathrm{i}\langle\boldsymbol{t}, d \boldsymbol{n}\rangle} \mathrm{d} \boldsymbol{t} \\
& =\frac{1}{(2 \pi)^{p}} \int_{2 \pi \mathbb{R}^{p} / \mathbb{Z}^{p}} \phi_{X-\boldsymbol{\mu}}^{n}(\boldsymbol{t}) e^{-\mathrm{i}\langle\boldsymbol{t}, d \boldsymbol{n}-n \boldsymbol{\mu}\rangle} \mathrm{d} \boldsymbol{t},
\end{aligned}
$$

where $\phi_{X}(\boldsymbol{t})$ and $\phi_{X-\boldsymbol{\mu}}(\boldsymbol{t})$ are the characteristic functions of $X$ and $X-\boldsymbol{\mu}$ respectively. We recall the domains $B_{j}(\delta)$ for $j=0,1,2, \cdots, p-1$ from (2.15). Thanks to Proposition 2.3, the characteristic function $\left|\phi_{X-\boldsymbol{\mu}}^{n}(\boldsymbol{t})\right|$ is exponentially small outside those sets $B_{j}(\delta)$.

$$
\begin{aligned}
\mathbb{P}\left(S_{n}=d \boldsymbol{n}\right) & =\frac{1}{(2 \pi)^{p}} \sum_{j=0}^{p-1} \int_{2 \pi B_{j}(\delta)} \phi_{X-\boldsymbol{\mu}}^{n}(\boldsymbol{t}) e^{-\mathrm{i}\langle\boldsymbol{t}, d \boldsymbol{n}-n \boldsymbol{\mu}\rangle} \mathrm{d} \boldsymbol{t}+e^{-c(\delta) n / p^{3}} \\
& =\frac{p}{(2 \pi)^{p}} \int_{2 \pi B_{0}(\delta)} \phi_{X-\boldsymbol{\mu}}^{n}(\boldsymbol{t}) e^{-\mathrm{i}\langle\boldsymbol{t}, d \boldsymbol{n}-n \boldsymbol{\mu}\rangle} \mathrm{d} \boldsymbol{t}+e^{-c(\delta) n / p^{3}},
\end{aligned}
$$

where we used the fact that the integrand is translation invariant by vectors $2 \pi(0,1 / p, 2 / p, \cdots, p-1 / p) \mathbb{Z}$. For any $\boldsymbol{t} \in B_{0}(\delta)$, by definition there exists $\boldsymbol{x} \in \mathbb{R}^{p-1}$ with $\|\boldsymbol{x}\|_{2}^{2} \leqslant \delta$ and $y \in[0,2 \sqrt{p} \pi]$, such that $\boldsymbol{t}=Q(\boldsymbol{x}, y)=O \boldsymbol{x}+(y / \sqrt{p}) \mathbf{1}$. By a change of variable, we can rewrite $(3.5)$ as

$$
\begin{aligned}
& \frac{p}{(2 \pi)^{p}} \int_{2 \pi B_{0}(\delta)} \phi_{X-\boldsymbol{\mu}}^{n}(\boldsymbol{t}) e^{-\mathrm{i}\langle\boldsymbol{t}, d \boldsymbol{n}-n \boldsymbol{\mu}\rangle} \mathrm{d} \boldsymbol{t} \\
= & \frac{p}{(2 \pi)^{p}} \int_{\left\{\boldsymbol{x} \in \mathbb{R}^{p-1}:\|\boldsymbol{x}\|_{2}^{2} \leqslant \delta\right\} \times[0,2 \sqrt{p} \pi]} \phi_{X-\boldsymbol{\mu}}^{n}(Q(\boldsymbol{x}, y)) e^{-\mathrm{i}\langle Q(\boldsymbol{x}, y), d \boldsymbol{n}-n \boldsymbol{\mu}\rangle} \mathrm{d} \boldsymbol{x} \mathrm{d} y \\
= & \frac{p^{3 / 2}}{(2 \pi)^{p-1}} \int_{\left\{\boldsymbol{x} \in \mathbb{R}^{p-1}:\|\boldsymbol{x}\|_{2}^{2} \leqslant \delta\right\}} \phi_{X-\boldsymbol{\mu}}^{n}(O \boldsymbol{x}) e^{-\mathrm{i}\langle O \boldsymbol{x}, d \boldsymbol{n}-n \boldsymbol{\mu}\rangle} \mathrm{d} \boldsymbol{x},
\end{aligned}
$$

where we used that $\langle\mathbf{1}, X-\boldsymbol{\mu}\rangle=0$ and $\langle\mathbf{1}, d \boldsymbol{n}-n \boldsymbol{\mu}\rangle=0$. By Taylor expansion, the characteristic function is

$$
\begin{aligned}
\phi_{X-\boldsymbol{\mu}}(O \boldsymbol{x}) & =\mathbb{E}\left[1+\mathrm{i}\langle O \boldsymbol{x}, X-\boldsymbol{\mu}\rangle-\frac{1}{2}\langle O \boldsymbol{x}, X-\boldsymbol{\mu}\rangle^{2}-\frac{\mathrm{i}}{6}\langle O \boldsymbol{x}, X-\boldsymbol{\mu}\rangle^{3}+\mathrm{O}\left(\langle O \boldsymbol{x}, X-\mu\rangle^{4}\right)\right] \\
& =1-\frac{1}{2} \boldsymbol{x}^{t} O^{t} \Sigma O \boldsymbol{x}+\mathrm{O}\left(\frac{\|\boldsymbol{x}\|_{2}^{3}}{p}\right)=1-\frac{d}{2 p}\|\boldsymbol{x}\|_{2}^{2}+\mathrm{O}\left(\frac{\|\boldsymbol{x}\|_{2}^{3}}{p}\right),
\end{aligned}
$$

where we used $\Sigma=d I_{p} / p-d \mathbf{1 1}^{t} / p^{2}$ from (2.13), and $O^{t} \Sigma O=d I_{p-1} / p$. Fix a large constant $\mathfrak{c}$, which will be chosen later. For $\mathfrak{c} p^{2} \ln n / n \leqslant\|\boldsymbol{x}\|_{2}^{2} \leqslant \delta$, we have

$$
\left|\phi_{X-\boldsymbol{\mu}}(O \boldsymbol{x})\right|^{n} \leqslant \exp \left\{-\left(\frac{\mathfrak{c} d}{2}+\mathrm{o}(1)\right) p \ln n\right\},
$$

which turns out to be negligible provided $\mathfrak{c}$ is large enough. In the following we will restrict the integral (3.6) on the domain $\left\{\boldsymbol{x} \in \mathbb{R}^{p-1}:\|\boldsymbol{x}\|_{2}^{2} \leqslant \mathfrak{c} p^{2} \ln n / n\right\}$. From $(3.7)$, on the domain $\left\{\boldsymbol{x} \in \mathbb{R}^{p-1}:\|\boldsymbol{x}\|_{2}^{2} \leqslant \mathfrak{c} p^{2} \ln n / n\right\}$, we have

$$
\phi_{X-\boldsymbol{\mu}}^{n}(O \boldsymbol{x})=\left(1+\mathrm{O}\left(\frac{p^{2}(\ln n)^{3 / 2}}{n^{1 / 2}}\right)\right) e^{-\frac{d n}{2 p}\|\boldsymbol{x}\|_{2}^{2}}
$$


and

$$
\begin{aligned}
& \frac{p^{3 / 2}}{(2 \pi)^{p-1}} \int_{\left\{\boldsymbol{x} \in \mathbb{R}^{p-1}:\|\boldsymbol{x}\|_{2}^{2} \leqslant \mathrm{c} p^{2} \ln n / n\right\}} \phi_{X-\boldsymbol{\mu}}^{n}(O \boldsymbol{x}) e^{-\mathrm{i}\langle O \boldsymbol{x}, d \boldsymbol{n}-n \boldsymbol{\mu}\rangle} \mathrm{d} \boldsymbol{x} \\
= & \left(1+\mathrm{O}\left(\frac{p^{2}(\ln n)^{3 / 2}}{n^{1 / 2}}\right)\right) \frac{p^{3 / 2}}{(2 \pi)^{p-1}} \int_{\left\{\boldsymbol{x} \in \mathbb{R}^{p-1}:\|\boldsymbol{x}\|_{2}^{2} \leqslant \mathrm{c} p^{2} \ln n / n\right\}} e^{-\frac{d n}{2 p}\|\boldsymbol{x}\|_{2}^{2}} e^{-\mathrm{i}\left\langle\boldsymbol{x}, O^{t}(d \boldsymbol{n}-n \boldsymbol{\mu})\right\rangle} \mathrm{d} \boldsymbol{x} \\
= & \left(1+\mathrm{O}\left(\frac{p^{2}(\ln n)^{3 / 2}}{n^{1 / 2}}\right)\right) \frac{p^{3 / 2}}{(2 \pi)^{p-1}} \int_{\mathbb{R}^{p-1}} e^{-\frac{d n}{2 p}\|\boldsymbol{x}\|_{2}^{2}} e^{-\mathrm{i}\left\langle\boldsymbol{x}, O^{t}(d \boldsymbol{n}-n \boldsymbol{\mu})\right\rangle} \mathrm{d} \boldsymbol{x}+e^{-\left(\frac{\mathrm{c} d}{2}+\mathrm{o}(1)\right) p \ln n} \\
= & \left(1+\mathrm{O}\left(\frac{p^{2}(\ln n)^{3 / 2}}{n^{1 / 2}}\right)\right) \frac{p^{3 / 2}}{(2 \pi)^{p-1}} \int_{\mathbb{R}^{p-1}} e^{-\frac{d n}{2 p}\|\boldsymbol{x}\|_{2}^{2}} e^{-\mathrm{i}\left\langle\boldsymbol{x}, O^{t}(d \boldsymbol{n}-n \boldsymbol{\mu})\right\rangle} \mathrm{d} \boldsymbol{x}+e^{-\left(\frac{\mathrm{c} d}{2}+\mathrm{o}(1)\right) p \ln n} \\
= & \left(1+\mathrm{O}\left(\frac{p^{2}(\ln n)^{3 / 2}}{n^{1 / 2}}\right)\right) p^{3 / 2}\left(\frac{p}{2 \pi d n}\right)^{\frac{p-1}{2}} e^{-\frac{d p n}{2}\left\|O^{t}\left(\frac{n}{n}-\frac{\mu}{d}\right)\right\|_{2}^{2}}+e^{-\left(\frac{\mathfrak{c} d}{2}+\mathrm{o}(1)\right) p \ln n} .
\end{aligned}
$$

The exponents in (3.4) and (3.9) cancel

$$
-\frac{d p n}{2}\left\|O^{t}\left(\frac{\boldsymbol{n}}{n}-\frac{\boldsymbol{\mu}}{d}\right)\right\|_{2}^{2}+\frac{(d-1) p n}{2} \sum_{j=0}^{p-1}\left(\mathfrak{n}_{j}-1 / p\right)^{2}=-\frac{p n}{2}\left\|O^{t}\left(\frac{\boldsymbol{n}}{n}-\frac{\boldsymbol{\mu}}{d}\right)\right\|_{2}^{2},
$$

where we used that $O^{t}$ is an isometry from $\left\{\left(x_{1}, x_{2}, \cdots, x_{p}\right) \in \mathbb{R}^{p}: x_{1}+x_{2}+\cdots+x_{p}=0\right\}$ to $\mathbb{R}^{p-1}$. Therefore, by combining the estimates (3.4), (3.5), (3.6), (3.8) and (3.9), we conclude that for any $p$-tuple $\left(n_{0}, n_{1}, \cdots, n_{p-1}\right) \in \mathcal{E}$, with $\sum_{j=0}^{p-1} j n_{j} \equiv 0(\bmod p)$,

$$
\begin{aligned}
& \frac{1}{\left|\mathrm{M}_{d, p}\right|} \sum_{\boldsymbol{v} \in \mathcal{S}\left(n_{0}, n_{1}, \cdots, n_{p-1}\right)}\left|\left\{\mathcal{G} \in \mathrm{M}_{n, d}: A(\mathcal{G}) \boldsymbol{v}=\mathbf{0}\right\}\right| \\
= & \left(1+\mathrm{O}\left(\frac{p^{2}(\ln n)^{3 / 2}}{n^{1 / 2}}\right)\right) p^{3 / 2}\left(\frac{p}{2 \pi n}\right)^{\frac{p-1}{2}} e^{-\frac{p n}{2}\left\|O^{t}\left(\frac{n}{n}-\frac{\mu}{d}\right)\right\|_{2}^{2}}+e^{-\left(\frac{c d}{2}-\frac{(d-1) \mathfrak{b}}{2}+\mathrm{o}(1)\right) p \ln n} .
\end{aligned}
$$

For the second term on the righthand side of (3.10), since the total number of $p$-tuples $\left(n_{0}, n_{1}, \cdots, n_{p-1}\right) \in \mathcal{E}$ is bounded by $e^{p \ln n}$,

$$
\sum_{\left(n_{0}, n_{1}, \cdots, n_{p-1}\right) \in \mathcal{E}} e^{-\left(\frac{\mathfrak{c} d}{2}-\frac{(d-1) \mathfrak{b}}{2}+\mathrm{o}(1)\right) p \ln n}=e^{-\left(\frac{\mathfrak{c} d}{2}-\frac{(d-1) \mathfrak{b}}{2}-1+\mathrm{o}(1)\right) p \ln n}
$$

which is negligible provided $\mathfrak{c}$ is large enough.

For the first term on the righthand side of (3.10) corresponding to the $p$-tuple $\left(n_{0}, n_{1}, \cdots, n_{p-1}\right) \in \mathcal{E}$, with $\sum_{j=0}^{p-1} j n_{j} \equiv 0(\bmod p)$, we can replace it by an average.

$$
p e^{-\frac{p n}{2}\left\|O^{t}\left(\frac{n}{n}-\frac{\mu}{d}\right)\right\|_{2}^{2}}=e^{-\frac{p n}{2}\left\|O^{t}\left(\frac{n}{n}-\frac{\mu}{d}\right)\right\|_{2}^{2}}+\left(1+\mathrm{O}\left(\frac{p(\ln n)^{1 / 2}}{n^{1 / 2}}\right)\right) \sum_{j=1}^{p-1} e^{-\frac{p n}{2}\left\|O^{t}\left(\frac{n+e_{j}-e_{0}}{n}-\frac{\mu}{d}\right)\right\|_{2}^{2}} .
$$

Therefore, we can replace the sum over $p$-tuples $\left(n_{0}, n_{1}, \cdots, n_{p-1}\right) \in \mathcal{E}$, with $\sum_{j=0}^{p-1} j n_{j} \equiv 0(\bmod p)$ to the 
sum over all $p$-tuples $\left(n_{0}, n_{1}, \cdots, n_{p-1}\right) \in \mathcal{E}$ with a factor $1 / p$.

$$
\begin{aligned}
& \sum_{\substack{\left(n_{0}, n_{1}, \cdots, n_{p-1}\right) \in \mathcal{E} \\
\sum_{j=0}^{p-1} j n_{j} \equiv 0(\bmod p)}}\left(1+\mathrm{O}\left(\frac{p^{2}(\ln n)^{3 / 2}}{n^{1 / 2}}\right)\right) p^{3 / 2}\left(\frac{p}{2 \pi n}\right)^{\frac{p-1}{2}} e^{-\frac{p n}{2}\left\|O^{t}\left(\frac{n}{n}-\frac{\mu}{d}\right)\right\|_{2}^{2}} \\
= & \sum_{\left(n_{0}, n_{1}, \cdots, n_{p-1}\right) \in \mathcal{E}}\left(1+\mathrm{O}\left(\frac{p^{2}(\ln n)^{3 / 2}}{n^{1 / 2}}\right)\right) p^{1 / 2}\left(\frac{p}{2 \pi n}\right)^{\frac{p-1}{2}} e^{-\frac{p n}{2}\left\|O^{t}\left(\frac{n}{n}-\frac{\mu}{d}\right)\right\|_{2}^{2} .}
\end{aligned}
$$

In the following we estimate the sum in (3.12). The set of points $O^{t}(\boldsymbol{n} / n-\boldsymbol{\mu} / d)$ for $\boldsymbol{n}=\left(n_{0}, n_{1}, \cdots, n_{p-1}\right) \in \mathcal{E}$ is a subset of a lattice in $\mathbb{R}^{p-1}$. A set of base for this lattice is given by

$$
O^{t}\left(e_{j}-e_{0}\right) / n, \quad 0 \leqslant j \leqslant p-1 .
$$

The volume of the fundamental domain is $p^{1 / 2} / n^{p-1}$. By viewing (3.12) as a Riemann sum, we can rewrite it as an integral on $\mathbb{R}^{p-1}$.

$$
\begin{aligned}
& \sum_{\left(n_{0}, n_{1}, \cdots, n_{p-1}\right) \in \mathcal{E}}\left(1+\mathrm{O}\left(\frac{p^{2}(\ln n)^{3 / 2}}{n^{1 / 2}}\right)\right) p^{1 / 2}\left(\frac{p}{2 \pi n}\right)^{\frac{p-1}{2}} e^{-\frac{p n}{2}\left\|O^{t}\left(\frac{n}{n}-\frac{\mu}{d}\right)\right\|_{2}^{2}} \\
= & \left(1+\mathrm{O}\left(\frac{p^{2}(\ln n)^{3 / 2}}{n^{1 / 2}}\right)\right)\left(\frac{p n}{2 \pi}\right)^{\frac{p-1}{2}} \int_{\left\{\boldsymbol{x} \in \mathbb{R}^{p-1}:\|\boldsymbol{x}\|_{2}^{2} \leqslant \mathfrak{b} \ln n / n\right\}} e^{-\frac{p n}{2}\|\boldsymbol{x}\|^{2}} \mathrm{~d} \boldsymbol{x} \\
= & \left(1+\mathrm{O}\left(\frac{p^{2}(\ln n)^{3 / 2}}{n^{1 / 2}}\right)\right),
\end{aligned}
$$

provided $\mathfrak{b}$ is large enough. The claim (3.2) follows from combining (3.10), (3.11) and (3.13). This finishes the proof of Proposition 3.1.

\subsection{Large deviation estimate}

In this section, we show that the sum of terms in (3.1) corresponding to non-equidistributed $p$-tuples $\left(n_{0}, n_{1}, \cdots, n_{p-1}\right)$ is small.

Proposition 3.2. Let $d \geqslant 3$ be a fixed integer, and a prime number $p$ such that $\operatorname{gcd}(p, d)=1$ and $p \ll$ $n^{(d-2) / 2 d}$. Then for $n$ sufficiently large,

$$
\frac{1}{\left|\mathrm{M}_{n, d}\right|} \sum_{\left(n_{0}, n_{1}, \cdots, n_{p-1}\right) \in \mathcal{N}} \sum_{\boldsymbol{v} \in \mathcal{S}\left(n_{0}, n_{1}, \cdots, n_{p-1}\right)}\left|\left\{\mathcal{G} \in \mathrm{M}_{n, d}: A(\mathcal{G}) \boldsymbol{v}=\mathbf{0}\right\}\right| \leqslant \frac{\mathrm{O}\left(p^{2}\right)}{n^{(d-2)}}
$$

Thanks to Proposition 2.1, we have

$$
\begin{aligned}
& \frac{1}{\left|\mathrm{M}_{n, d}\right|} \sum_{\left(n_{0}, n_{1}, \cdots, n_{p-1}\right) \in \mathcal{N} \boldsymbol{v} \in \mathcal{S}\left(n_{0}, n_{1}, \cdots, n_{p-1}\right)}\left|\left\{\mathcal{G} \in \mathrm{M}_{n, d}: A(\mathcal{G}) \boldsymbol{v}=\mathbf{0}\right\}\right| \\
= & \sum_{\left(n_{0}, n_{1}, \cdots, n_{p-1}\right) \in \mathcal{N}}\left(\begin{array}{c}
n \\
n_{0}, n_{1}, \cdots, n_{p-1}
\end{array}\right)\left(\begin{array}{c}
d n \\
d n_{0}, d n_{1}, \cdots, d n_{p-1}
\end{array}\right)^{-1} \\
& \times p^{n(d-1)} \mathbb{P}\left(X_{1}+X_{2}+\cdots+X_{n}=\left(d n_{0}, d n_{1}, \cdots, d n_{p-1}\right)\right),
\end{aligned}
$$


where $X_{1}, X_{2}, \cdots, X_{n}$ are independent copies of $X$, which is uniform distributed over $\mathcal{U}_{d, p}$ as defined in (2.1). We enumerate the elements of $\mathcal{U}_{d, p}$ as

$$
\mathcal{U}_{d, p}=\left\{\boldsymbol{w}_{1}, \boldsymbol{w}_{2}, \cdots, \boldsymbol{w}_{p^{d-1}}\right\}, \quad \boldsymbol{w}_{1}=(d, 0,0, \cdots, 0) .
$$

For any non-equidistributed $p$-tuple $\left(n_{0}, n_{1}, \cdots, n_{p-1}\right)$, we denote $\mathfrak{n}_{j}=n_{j} / n$ for $j=0,1, \cdots, p-1$. We estimate the first factor on the righthand side of (3.15) using Stirling's formula,

$$
\left(\begin{array}{c}
n \\
n_{0}, n_{1}, \cdots, n_{p-1}
\end{array}\right)\left(\begin{array}{c}
d n \\
d n_{0}, d n_{1}, \cdots, d n_{p-1}
\end{array}\right)^{-1} p^{n(d-1)} \leqslant e^{\mathrm{O}(p)} \exp \left\{(d-1) n\left(\ln p+\sum_{j=0}^{p-1} \mathfrak{n}_{j} \ln \mathfrak{n}_{j}\right)\right\}
$$

For the random walk term in (3.15), we have the following large deviation bound

$$
\mathbb{P}\left(X_{1}+X_{2}+\cdots+X_{n}=\left(d n_{0}, d n_{1}, \cdots, d n_{p-1}\right)\right) \leqslant \exp \left\{n \inf _{\boldsymbol{t} \in \mathbb{R}^{p}} \log \mathbb{E}\left[e^{\langle\boldsymbol{t}, X\rangle}\right]-d\langle\boldsymbol{t}, \mathfrak{n}\rangle\right\} .
$$

Thus combining (3.16) and (3.17), we get that

$$
\begin{aligned}
& \frac{1}{\left|\mathrm{M}_{n, d}\right|} \sum_{\left(n_{0}, n_{1}, \cdots, n_{p-1}\right) \in \mathcal{N}} \sum_{\boldsymbol{v} \in \mathcal{S}\left(n_{0}, n_{1}, \cdots, n_{p-1}\right)}\left|\left\{\mathcal{G} \in \mathrm{M}_{n, d}: A(\mathcal{G}) \boldsymbol{v}=\mathbf{0}\right\}\right| \\
\leqslant & \sum_{\left(n_{0}, n_{1}, \cdots, n_{p-1}\right) \in \mathcal{N}} e^{\mathrm{O}(p)} \exp \left\{(d-1) n \ln p+(d-1) n \sum_{j=0}^{p-1} \mathfrak{n}_{j} \ln \mathfrak{n}_{j}+n \inf _{\boldsymbol{t} \in \mathbb{R}^{p}} \log \mathbb{E}\left[e^{\langle\boldsymbol{t}, X\rangle}\right]-d\langle\boldsymbol{t}, \mathfrak{n}\rangle\right\} \\
= & \sum_{\left(n_{0}, n_{1}, \cdots, n_{p-1}\right) \in \mathcal{N}} e^{\mathrm{O}(p)} e^{n I\left(\mathfrak{n}_{0}, \mathfrak{n}_{1}, \cdots, \mathfrak{n}_{p-1}\right)},
\end{aligned}
$$

where the rate function is given by

$$
I\left(\mathfrak{n}_{0}, \mathfrak{n}_{1}, \cdots, \mathfrak{n}_{p-1}\right)=(d-1) \ln p+(d-1) \sum_{j=0}^{p-1} \mathfrak{n}_{j} \ln \mathfrak{n}_{j}+\inf _{\boldsymbol{t} \in \mathbb{R}^{p}} \log \mathbb{E}\left[e^{\langle\boldsymbol{t}, X\rangle}\right]-d\langle\boldsymbol{t}, \mathfrak{n}\rangle .
$$

The rate function function is negative except for two points: $\mathfrak{n}_{0}=\mathfrak{n}_{1}=\cdots=\mathfrak{n}_{p-1}=1 / p$, and $\mathfrak{n}_{0}=1$, $\mathfrak{n}_{1}=\cdots=\mathfrak{n}_{p-1}=0$. In the following proposition we give a quantitative estimate of the rate function.

Proposition 3.3. Let $d \geqslant 3$ be a fixed integer, and a prime number $p$ such that $\operatorname{gcd}(p, d)=1$. The rate function as defined in (3.18) satisfies: for any small $\delta>0$, there exists a constant $c(\delta)>0$, such that

$$
I\left(\mathfrak{n}_{0}, \mathfrak{n}_{1}, \cdots, \mathfrak{n}_{p-1}\right) \leqslant-\frac{c(\delta)}{p},
$$

unless $\max _{0 \leqslant k \leqslant p}\left|\mathfrak{n}_{k}-1 / p\right| \leqslant \delta / p$, or $\mathfrak{n}_{0} \geqslant 1-\delta / p$.

Proof. We take $\boldsymbol{t}=(d-1) / d\left(\left(\ln \mathfrak{n}_{0}, \ln \mathfrak{n}_{1}, \cdots, \ln \mathfrak{n}_{p-1}\right)+\ln p\right)$ in (3.18), the rate function $I$ is upper bounded by

$$
I\left(\mathfrak{n}_{0}, \mathfrak{n}_{1}, \cdots, \mathfrak{n}_{p-1}\right) \leqslant \log \sum_{j=1}^{p^{d-1}} \prod_{k=0}^{p-1} \mathfrak{n}_{k}^{\frac{d-1}{d} \boldsymbol{w}_{j}(k)}
$$


In the following, we prove that there exists a constant $c(\delta)>0$

$$
\sum_{j=1}^{p^{d-1}} \prod_{k=0}^{p-1} \mathfrak{n}_{k}^{\frac{d-1}{d} \boldsymbol{w}_{j}(k)} \leqslant 1-\frac{c(\delta)}{p},
$$

unless $\max _{0 \leqslant k \leqslant p}\left|\mathfrak{n}_{k}-1 / p\right| \leqslant \delta / p$, or $\mathfrak{n}_{0} \geqslant 1-\delta / p$. Then the claim (3.19) follows.

For any $\varepsilon>0$ and $d$-tuple $\left(a_{1}, a_{2}, \cdots, a_{d}\right) \in \mathbb{F}_{p}^{d}$ such that $a_{1}+a_{2}+\cdots+a_{d}=0$, if

$$
\frac{\min _{1 \leqslant r \leqslant d} \mathfrak{n}_{a_{r}}}{\max _{1 \leqslant r \leqslant d} \mathfrak{n}_{a_{r}}} \leqslant \frac{1}{1+\varepsilon},
$$

then there exists a constant $c(\varepsilon)>0$ such that

$$
\prod_{r=1}^{d} \mathfrak{n}_{a_{r}}^{\frac{d-1}{d}} \leqslant \frac{1-c(\varepsilon)}{d} \sum_{r=1}^{d} \prod_{\substack{1 \leqslant s \leqslant d \\ s \neq r}} \mathfrak{n}_{a_{s}} .
$$

Therefore, by the defining relation of the multiset $\mathcal{U}_{d, p}$ as in (2.1), we have

$$
\begin{aligned}
& \sum_{j=1}^{p^{d-1}} \prod_{k=0}^{p-1} \mathfrak{n}_{k}^{\frac{d-1}{d} \boldsymbol{w}_{j}(k)}=\sum_{\substack{\left(a_{1}, a_{2}, \cdots, a_{d}\right) \in \mathbb{F}_{p}^{d}, r=1 \\
a_{1}+a_{2}+\cdots+a_{d}=\mathbf{0}}} \prod_{\substack{d \\
\mathfrak{n}_{r}}}^{\frac{d-1}{d}} \\
& \leqslant \sum_{\substack{\left(a_{1}, a_{2}, \cdots, a_{d}\right) \in \mathbb{F}_{p}^{d}, a_{1}+a_{2}+\cdots+a_{d}=\mathbf{0}}} \frac{1}{d}\left(1-1_{\frac{\min _{r} n_{a_{r}}}{\max _{r} \mathbf{n}_{a_{r}}} \leqslant \frac{1}{1+\varepsilon}} c(\varepsilon)\right) \sum_{r=1}^{d} \prod_{\substack{1 \leqslant s \leqslant d \\
s \neq r}} \mathfrak{n}_{a_{s}} \\
& =\left(\sum_{j=0}^{p-1} \mathfrak{n}_{j}\right)^{d-1}-\frac{c(\varepsilon)}{d} \sum_{\substack{\left(a_{1}, a_{2}, \cdots, a_{d}\right) \in \mathbb{F}_{p}^{d}, a_{1}+a_{2}+\cdots+a_{d}=\mathbf{0}}} 1 \frac{\min _{r} \mathfrak{n}_{a_{r}}}{\max _{r} \mathfrak{n}_{a_{r}}} \leqslant \frac{1}{1+\varepsilon} \sum_{r=1}^{d} \prod_{\substack{1 \leqslant s \leqslant d \\
s \neq r}} \mathfrak{n}_{a_{s}} \\
& \leqslant 1-\frac{c(\varepsilon)}{d} \sum_{\substack{\left(a_{1}, a_{2}, \cdots, a_{d}\right) \in \mathbb{P}_{p}, a_{1}+a_{2}+\cdots+a_{d}=\mathbf{0}}} 1_{\frac{\min _{r} \mathfrak{n}_{a_{r}}}{\max \mathfrak{n}_{r} a_{r}} \leqslant \frac{1}{1+\varepsilon}} \prod_{r=1}^{d-1} \mathfrak{n}_{a_{r}} .
\end{aligned}
$$

In the following, we take $\varepsilon=\delta / 3$, and prove that there exists a constant $c(\delta)$

$$
\sum_{\substack{\left(a_{1}, a_{2}, \cdots, a_{d}\right) \in \mathbb{F}_{p}^{d} \\ a_{1}+a_{2}+\cdots+a_{d}=\mathbf{0}}} 1_{\frac{\min _{r} \mathfrak{n}_{a_{r}}}{\max \mathfrak{n}_{r}} \leqslant \frac{1}{1+\varepsilon}} \prod_{r=1}^{d-1} \mathfrak{n}_{a_{r}} \geqslant \frac{c(\delta)}{p},
$$

unless $\max _{0 \leqslant k \leqslant p}\left|\mathfrak{n}_{k}-1 / p\right| \leqslant \delta / p$, or $\mathfrak{n}_{0} \geqslant 1-\delta / p$. Then the claim (3.21) follows.

We sort these numbers $\mathfrak{n}_{0}, \mathfrak{n}_{1}, \cdots, \mathfrak{n}_{p-1}$ as $\mathfrak{n}_{k_{1}} \geqslant \mathfrak{n}_{k_{2}} \geqslant \cdots \geqslant \mathfrak{n}_{k_{p}}$, then $\mathfrak{n}_{k_{1}} \geqslant 1 / p$. We take the indices $t_{1}$ and $t_{2}$ such that $\mathfrak{n}_{k_{t_{1}}}>\mathfrak{n}_{k_{1}} /(1+\varepsilon) \geqslant \mathfrak{n}_{k_{t_{1}+1}}$, and $\mathfrak{n}_{k_{t_{2}}}>\mathfrak{n}_{k_{1}} /(1+\varepsilon)^{2} \geqslant \mathfrak{n}_{k_{t_{2}+1}}$. If $\mathfrak{n}_{k_{t_{1}+1}}+\mathfrak{n}_{k_{t_{1}+2}}+\cdots+\mathfrak{n}_{k_{p}} \geqslant \varepsilon$, 
by restricting the sum in (3.23) over $d$-tuples with $a_{1}=k_{1}$ and $a_{2} \in\left\{k_{t_{1}+1}, k_{t_{1}+2}, \cdots, k_{p}\right\}$, we get

$$
\sum_{\substack{\left(a_{1}, a_{2}, \cdots, a_{d}\right) \in \mathbb{F}_{p}^{d}, a_{1}+a_{2}+\cdots+a_{d}=\mathbf{0}}} 1_{\frac{\min _{r} \mathfrak{n}_{a_{r}}}{\max \mathfrak{n}_{a_{r}}} \leqslant \frac{1}{1+\varepsilon}} \sum_{r=1}^{d-1} \mathfrak{n}_{a_{r}} \geqslant \mathfrak{n}_{k_{1}}\left(\mathfrak{n}_{k_{t_{1}+1}}+\mathfrak{n}_{k_{t_{1}+2}}+\cdots+\mathfrak{n}_{k_{p}}\right) \sum_{a_{3}, a_{4}, \cdots, a_{d-1} \in \mathbb{F}_{p}} \prod_{r=3}^{d-1} \mathfrak{n}_{a_{r}} \geqslant \frac{\varepsilon}{p}
$$

The claim (3.23) follows. So we can assume that $\mathfrak{n}_{k_{t_{1}+1}}+\mathfrak{n}_{k_{t_{1}+2}}+\cdots+\mathfrak{n}_{k_{p}} \leqslant \varepsilon$ and thus $\mathfrak{n}_{k_{1}}+\mathfrak{n}_{k_{2}}+$ $\cdots+\mathfrak{n}_{k_{t_{1}}} \geqslant 1-\varepsilon$. If $\mathfrak{n}_{k_{t_{2}+1}}+\mathfrak{n}_{k_{t_{2}+2}}+\cdots+\mathfrak{n}_{k_{p}} \geqslant \varepsilon / p$, by restricting the sum in (3.23) over $d$-tuples with $a_{1} \in\left\{k_{1}, k_{2}, \cdots, k_{t_{1}}\right\}$ and $a_{2} \in\left\{k_{t_{2}+1}, k_{t_{2}+2}, \cdots, k_{p}\right\}$, we get

$$
\begin{gathered}
\sum_{\substack{\left(a_{1}, a_{2}, \cdots, a_{d}\right) \in \mathbb{F}_{p}^{d}, a_{1}+a_{2}+\cdots+a_{d}=0}} 1_{\frac{\min _{r} \mathfrak{n}_{a_{r}}}{\max _{r} \mathfrak{n}_{a_{r}}} \leqslant \frac{1}{1+\varepsilon}} \sum_{r=1}^{d-1} \mathfrak{n}_{a_{r}} \\
\geqslant\left(\mathfrak{n}_{k_{1}}+\mathfrak{n}_{k_{2}}+\cdots+\mathfrak{n}_{k_{t_{1}}}\right)\left(\mathfrak{n}_{k_{t_{2}+1}}+\mathfrak{n}_{k_{t_{2}+2}}+\cdots+\mathfrak{n}_{k_{p}}\right) \sum_{a_{3}, a_{4}, \cdots, a_{d-1} \in \mathbb{F}_{p}} \prod_{r=3}^{d-1} \mathfrak{n}_{a_{r}} \geqslant \frac{(1-\varepsilon) \varepsilon}{p} .
\end{gathered}
$$

The claim (3.23) follows. So we can assume that $\mathfrak{n}_{k_{t_{2}+1}}+\mathfrak{n}_{k_{t_{2}+2}}+\cdots+\mathfrak{n}_{k_{p}} \leqslant \varepsilon / p$. There are three cases:

1. If $t_{2}=p$, then we have $\max _{0 \leqslant k \leqslant p-1} \mathfrak{n}_{k} \leqslant(1+\varepsilon)^{2} \min _{0 \leqslant k \leqslant p-1} \mathfrak{n}_{k}$. And it follows that $(1+\varepsilon)^{-2} / p \leqslant$ $\mathfrak{n}_{k} \leqslant(1+\varepsilon)^{2} / p$ for $0 \leqslant k \leqslant p-1$. The claim follows by taking $2 \varepsilon+\varepsilon^{2} \leqslant \delta$.

2. If $t_{2}=1$, then we have $\mathfrak{n}_{k_{1}} \geqslant 1-\varepsilon / p$. If $k_{1}=0$, then the claim follows by taking $\varepsilon \leqslant \delta$. Otherwise, $k_{1} \neq 0$. By restricting the sum in (3.23) over $d$-tuples with $a_{1}=a_{2}=\cdots=a_{d-1}=k_{1}$, we get

$$
\sum_{\substack{\left(a_{1}, a_{2}, \cdots, a_{d}\right) \in \mathbb{F}_{p}^{d}, a_{1}+a_{2}+\cdots+a_{d}=\mathbf{0}}} 1_{\frac{\min _{r} \mathfrak{n}_{a_{r}}}{\max _{r} \mathfrak{n}_{a_{r}}} \leqslant \frac{1}{1+\varepsilon}} \sum_{r=1}^{d-1} \mathfrak{n}_{a_{r}} \geqslant \mathfrak{n}_{k_{1}}^{d-1} \geqslant\left(1-\frac{\varepsilon}{p}\right)^{d-1}
$$

The claim (3.23) follows.

3. If $1<t_{2}<p$, then we have $\mathfrak{n}_{k_{1}} \geqslant \mathfrak{n}_{k_{2}} \geqslant \cdots \geqslant \mathfrak{n}_{k_{t_{2}}} \geqslant \mathfrak{n}_{k_{1}} /(1+\varepsilon)^{2}$. And it follows $(1+\varepsilon)^{2} / t_{2} \geqslant$ $\mathfrak{n}_{k_{1}} \geqslant \mathfrak{n}_{k_{2}} \geqslant \cdots \geqslant \mathfrak{n}_{k_{t_{2}}} \geqslant(1+\varepsilon)^{-2} / t_{2} \geqslant(1+\varepsilon) \mathfrak{n}_{k_{t_{2}}+1}$. We will restrict the sum in (3.23) over $d$-tuples with $a_{1}, a_{2}, \cdots, a_{d-1} \in\left\{k_{1}, k_{2}, \cdots, k_{t_{2}}\right\}$, and $a_{d} \in\left\{k_{t_{2}+1}, k_{t_{2}+2}, \cdots, k_{p}\right\}$. We take integer $q$ such that $t_{2} q \equiv-2\left(k_{1}+k_{2}+\cdots+k_{t_{2}}\right)(\bmod p)$. The number of $(d-2)$-tuples $a_{1}, a_{2}, \cdots, a_{d-2} \in$ $\left\{k_{1}, k_{2}, \cdots, k_{t_{2}}\right\}$ such that $a_{1}+a_{2}+\cdots+a_{d-2} \not \equiv q(\bmod p)$ is at least $\left(t_{2}-1\right) t_{2}^{d-3}$. For any $(d-2)$-tuple $a_{1}, a_{2}, \cdots, a_{d-2} \in\left\{k_{1}, k_{2}, \cdots, k_{t_{2}}\right\}$ such that $a_{1}+a_{2}+\cdots+a_{d-2} \not \equiv q(\bmod p)$, there exists at least one $a_{d-1} \in\left\{k_{1}, k_{2}, \cdots, k_{t_{2}}\right\}$ such that $a_{1}+a_{2}+\cdots+a_{d-1} \not \equiv-k_{1},-k_{2}, \cdots,-k_{t_{2}}(\bmod p)$. Otherwise

$$
t_{2}\left(a_{1}+a_{2}+\cdots+a_{d-2}\right)+\left(k_{1}+k_{2}+\cdots+k_{t_{2}}\right) \equiv-\left(k_{1}+k_{2}+\cdots+k_{t_{2}}\right)(\bmod p),
$$

and $a_{1}+a_{2}+\cdots+a_{d-2} \equiv q(\bmod p)$. This leads to a contradiction. Therefore, the number of $d-$ tuples with $a_{1}, a_{2}, \cdots, a_{d-1} \in\left\{k_{1}, k_{2}, \cdots, k_{t_{2}}\right\}$, and $a_{d} \in\left\{k_{t_{2}+1}, k_{t_{2}+2}, \cdots, k_{p}\right\}$ is at least $\left(t_{2}-1\right) t_{2}^{d-3}$. By restricting the sum in (3.23) over $d$-tuples with $a_{1}, a_{2}, \cdots, a_{d-1} \in\left\{k_{1}, k_{2}, \cdots, k_{t_{2}}\right\}$, and $a_{d} \in$ $\left\{k_{t_{2}+1}, k_{t_{2}+2}, \cdots, k_{p}\right\}$, we get

$$
\sum_{\substack{\left(a_{1}, a_{2}, \cdots, a_{d}\right) \in \mathbb{F}_{p}^{d}, a_{1}+a_{2}+\cdots+a_{d}=\mathbf{0}}} 1 \frac{1}{\frac{\min _{r} \mathfrak{n}_{a_{r}}}{\max _{r} \mathfrak{n}_{r}} \leqslant \frac{1}{1+\varepsilon}} \sum_{r=1}^{d-1} \mathfrak{n}_{a_{r}} \geqslant\left(t_{2}-1\right) t_{2}^{d-3}\left(\frac{1}{(1+\varepsilon)^{2} t_{2}}\right)^{d-1} \geqslant \frac{1}{2(1+\varepsilon)^{2 d-2} p} .
$$


The claim (3.23) follows.

This finishes the proof of Proposition 3.3.

Proof of Proposition 4.2. We further decompose the set of non-equidistributed $p$-tuples $\left(n_{0}, n_{1}, \cdots, n_{p-1}\right)$ into four classes:

1. $p$-tuples $\left(n_{0}, n_{1}, \cdots, n_{p-1}\right) \in \mathcal{N}$ with $\max _{0 \leqslant j \leqslant p-1}\left|n_{j} / n-1 / p\right| \leqslant \delta / p$.

2. $p$-tuples $\left(n_{0}, n_{1}, \cdots, n_{p-1}\right) \in \mathcal{N}$ with $\mathfrak{b} \ln n / n<\left|n_{0} / n-1\right| \leqslant \delta / p$.

3. $p$-tuples $\left(n_{0}, n_{1}, \cdots, n_{p-1}\right) \in \mathcal{N}$ with $\left|n_{0} / n-1\right| \leqslant \mathfrak{b} \ln n / n$.

4. The remaining non-equidistributed $p$-tuples.

For the first class, $\max _{0 \leqslant j \leqslant p-1}\left|n_{j} / n-1 / p\right| \leqslant \delta / p$. The total number of such $p$-tuples is $e^{\mathrm{O}(p \ln n)}$. Given a $p$-tuple $\left(n_{0}, n_{1}, \cdots, n_{p-1}\right)$ in the first class, we will derive a more precise estimate of (3.21), by a perturbation argument. Let

$$
\mathfrak{n}_{j}=\left(1+\delta_{j}\right) / p, \quad j=0,1, \cdots, p-1 .
$$

where $\sum_{0 \leqslant j \leqslant p-1} \delta_{j}=0, \max _{0 \leqslant j \leqslant p-1}\left|\delta_{j}\right| \leqslant \delta$, and $\sum_{j} \delta_{j}^{2} \geqslant \mathfrak{b} p^{2} \ln n / n$. We denote $\boldsymbol{\delta}=\left(\delta_{0}, \delta_{1}, \cdots, \delta_{p-1}\right)$. We use Taylor expansion, and rewrite the righthand side of (3.20) as

$$
\begin{aligned}
& \left.\sum_{j=1}^{p^{d-1}} \prod_{k=0}^{p-1} \mathfrak{n}_{k}^{\frac{d-1}{d}} \boldsymbol{w}_{j}(k)=\frac{1}{p^{d-1}} \sum_{j=1}^{p^{d-1}} \prod_{k=0}^{p-1}\left(1+\delta_{k}\right)^{\frac{d-1}{d}} \boldsymbol{w}_{j}(k)=\frac{1}{p^{d-1}} \sum_{j=1}^{p^{d-1}} e^{\frac{d-1}{d} \sum_{k=0}^{p-1}\left(\delta_{k}-(1+\mathrm{O}(\delta)) \frac{\delta_{k}^{2}}{2}\right.}\right)^{\boldsymbol{w}_{j}(k)} \\
= & \frac{1}{p^{d-1}} \sum_{j=1}^{p^{d-1}} 1+\frac{d-1}{d} \sum_{k=0}^{p-1}\left(\delta_{k}-(1+\mathrm{O}(\delta)) \frac{\delta_{k}^{2}}{2}\right) \boldsymbol{w}_{j}(k)+(1+\mathrm{O}(\delta)) \frac{(d-1)^{2}}{2 d^{2}}\left(\sum_{k=0}^{p-1} \delta_{k} \boldsymbol{w}_{j}(k)\right)^{2} \\
= & 1+\frac{d-1}{2 d p^{d-1}}\left(-(1+\mathrm{O}(\delta)) d p^{d-2}\|\boldsymbol{\delta}\|_{2}^{2}+(1+\mathrm{O}(\delta)) \frac{d-1}{d} \sum_{j=1}^{p^{d-1}}\left\langle\boldsymbol{\delta}, \boldsymbol{w}_{j}\right\rangle^{2}\right)
\end{aligned}
$$

We recall from (2.12) that

$$
\sum_{j=1}^{p^{d-1}} \boldsymbol{w}_{j} \boldsymbol{w}_{j}^{t}=d p^{d-2} I_{p}+d(d-1) p^{d-3} \mathbf{1 1}^{t}
$$

Therefore,

$$
\begin{aligned}
\sum_{j=1}^{p^{d-1}} \prod_{k=0}^{p-1} \mathfrak{n}_{k}^{\frac{d-1}{d} \boldsymbol{w}_{j}(k)} & =1+\frac{d-1}{2 d p^{d-1}}\left(-(1+\mathrm{O}(\delta)) d p^{d-2}\|\boldsymbol{\delta}\|_{2}^{2}+(1+\mathrm{O}(\delta))(d-1) p^{d-2}\|\boldsymbol{\delta}\|_{2}^{2}\right) \\
& =1-(1+\mathrm{O}(\delta)) \frac{d-1}{2 d p}\|\boldsymbol{\delta}\|_{2}^{2} \leqslant 1-(1+\mathrm{O}(\delta)) \frac{d-1}{2 d} \frac{\mathfrak{b} p \ln n}{n}
\end{aligned}
$$

Thanks to (3.20), we obtain an upper bound for the rate function from (3.25)

$$
I\left(\mathfrak{n}_{0}, \mathfrak{n}_{1}, \cdots, \mathfrak{n}_{p-1}\right) \leqslant-\left(\frac{(d-1) \mathfrak{b}}{2 d}+\mathrm{o}(1)\right) \frac{p \ln n}{n} .
$$


The total contribution of terms in (3.14) satisfying $\max _{0 \leqslant j \leqslant p-1}\left|n_{j} / n-1 / p\right| \leqslant \delta$ is bounded by

$$
\exp \left\{-\left(\frac{(d-1) \mathfrak{b}}{2 d}+\mathrm{o}(1)\right) p \ln n+\mathrm{O}(p \ln n)\right\}=\frac{\mathrm{o}(1)}{n^{(d-2)}}
$$

provided that we take $\mathfrak{b}$ sufficiently large.

For the second class, $\mathfrak{b} p \ln n / n<\left|n_{0} / n-1\right| \leqslant \delta / p$. The total number of such $p$-tuples is $e^{\mathrm{O}(p \ln n)}$. Given a $p$-tuple $\left(n_{0}, n_{1}, \cdots, n_{p-1}\right)$ in the second class, we will derive a more precise estimate of (3.21), by a perturbation argument. Let $\mathfrak{n}_{0}=1-\delta_{0}$, where $\delta_{0} \leqslant \delta / p$.

We decompose the $d$-tuples $\left(a_{1}, a_{2}, \cdots, a_{d}\right) \in \mathbb{F}_{p}^{d}$ such that $a_{1}+a_{2}+\cdots+a_{d}=0$ into three sets: $\{(0,0, \cdots, 0)\}$ and

$$
\begin{aligned}
& \mathcal{A}_{1}=\left\{\left(a_{1}, a_{2}, \cdots, a_{d}\right) \in \mathbb{F}_{p}^{d}: a_{1}+a_{2}+\cdots+a_{d}=0, \sum_{r=1}^{d} \mathbf{1}\left(a_{r}=0\right)=0\right\}, \\
& \mathcal{A}_{2}=\left\{\left(a_{1}, a_{2}, \cdots, a_{d}\right) \in \mathbb{F}_{p}^{d}: a_{1}+a_{2}+\cdots+a_{d}=0,0<\sum_{r=1}^{d} \mathbf{1}\left(a_{r}=0\right)<d\right\} .
\end{aligned}
$$

For any $d$-tuple $\left(a_{1}, a_{2}, \cdots, a_{d}\right) \in \mathcal{A}_{2}$, we have $\min _{1 \leqslant r \leqslant d} \mathfrak{n}_{a_{r}} / \max _{1 \leqslant r \leqslant d} \mathfrak{n}_{a_{r}} \leqslant \delta_{0} /\left(1-\delta_{0}\right)$, and

$$
\prod_{r=1}^{d} \mathfrak{n}_{a_{r}}^{\frac{d-1}{d}} \leqslant\left(4 \delta_{0}\right)^{1 / d} \frac{1}{d} \sum_{r=1}^{d} \prod_{\substack{1 \leqslant s \leqslant d \\ s \neq r}} \mathfrak{n}_{a_{s}} .
$$

Therefore, by the defining relation of the multiset $\mathcal{U}_{d, p}$ as in (2.1), we have

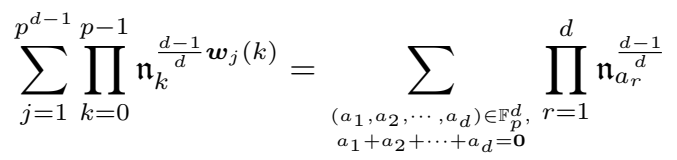

$$
\begin{aligned}
& \leqslant \mathfrak{n}_{0}^{d-1}+\sum_{\left(a_{1}, a_{2}, \cdots, a_{d}\right) \in \mathcal{A}_{1}} \frac{1}{d} \sum_{r=1}^{d} \prod_{\substack{1 \leqslant s \leqslant d \\
s \neq r}} \mathfrak{n}_{a_{s}}+\left(4 \delta_{0}\right)^{1 / d} \sum_{\left(a_{1}, a_{2}, \cdots, a_{d}\right) \in \mathcal{A}_{2}} \frac{1}{d} \sum_{r=1}^{d} \prod_{\substack{1 \leqslant s \leqslant d \\
s \neq r}} \mathfrak{n}_{a_{s}} \\
& \leqslant \mathfrak{n}_{0}^{d-1}+\left(\mathfrak{n}_{1}+\mathfrak{n}_{2}+\cdots+\mathfrak{n}_{p-1}\right)^{d-1}+\left(4 \delta_{0}\right)^{1 / d}\left(1-\mathfrak{n}_{0}^{d-1}\right) \\
& =1+\delta_{0}^{d-1}-\left(1-\left(4 \delta_{0}\right)^{1 / d}\right)\left(1-\left(1-\delta_{0}\right)^{d-1}\right) \text {. }
\end{aligned}
$$

Therefore, thanks to (3.20), we get

$$
I\left(\mathfrak{n}_{0}, \mathfrak{n}_{1}, \cdots, \mathfrak{n}_{p-1}\right) \leqslant-(1+\mathrm{o}(1))(d-1) \delta_{0}
$$

provided that $\delta_{0}$ is small enough. Thus, the total contribution of terms in (3.14) satisfying $\mathfrak{b} p \ln n / n<$ $\left|n_{0} / n-1\right| \leqslant \delta / p$ is bounded by

$$
\exp \{-(1+\mathrm{o}(1)) \mathfrak{b}(d-1) p \ln n+\mathrm{O}(p \ln n)\}=\frac{\mathrm{o}(1)}{n^{(d-2)}}
$$


provided that we take $\mathfrak{b}$ sufficiently large.

For the third class, $\left|n_{0} / n-1\right| \leqslant \mathfrak{b} p \ln n / n$. We rewrite (3.15) in terms of number of walk paths,

$$
\begin{gathered}
\sum_{\left(n_{0}, n_{1}, \cdots, n_{p-1}\right) \in \mathcal{N}}\left(\begin{array}{c}
n \\
n_{0}, n_{1}, \cdots, n_{p-1}
\end{array}\right)\left(\begin{array}{c}
d n \\
d n_{0}, d n_{1}, \cdots, d n_{p-1}
\end{array}\right)^{-1} \\
\times\left|\left\{\left(\boldsymbol{u}_{1}, \boldsymbol{u}_{2} \cdots, \boldsymbol{u}_{n}\right) \in \mathcal{U}_{d, p}^{n}: \boldsymbol{u}_{1}+\boldsymbol{u}_{2}+\cdots+\boldsymbol{u}_{n}=\left(d n_{0}, d n_{1}, \cdots, d n_{p-1}\right)\right\}\right| .
\end{gathered}
$$

Given a $p$-tuple $\left(n_{0}, n_{1}, \cdots, n_{p-1}\right)$, in the third class with $n_{0}=n-m$ and $2 \leqslant m \leqslant \mathfrak{b} p \ln n$, we reestimate the first factor on the righthand side of (3.29),

$$
\left(\begin{array}{c}
n \\
n_{0}, n_{1}, \cdots, n_{p-1}
\end{array}\right)\left(\begin{array}{c}
d n \\
d n_{0}, d n_{1}, \cdots, d n_{p-1}
\end{array}\right)^{-1} \leqslant \frac{e^{\mathrm{O}(m)}}{n^{(d-1) m}} \frac{\left(d n_{1}\right) !\left(d n_{2}\right) ! \cdots\left(d n_{p-1}\right) !}{n_{1} ! n_{2} ! \cdots n_{p-1} !}
$$

For the number of walk paths in (3.29), we recall that

$$
\mathcal{U}_{d, p}=\left\{\boldsymbol{w}_{1}, \boldsymbol{w}_{2}, \cdots, \boldsymbol{w}_{p^{d-1}}\right\}, \quad \boldsymbol{w}_{1}=(d, 0,0, \cdots, 0),
$$

and notice that $\boldsymbol{w}_{j}(1)+\boldsymbol{w}_{j}(2)+\cdots \boldsymbol{w}_{j}(p-1) \geqslant 2$ for $2 \leqslant j \leqslant p^{d-1}$. Moreover, since $n_{0}=n-m$, we have $\boldsymbol{u}_{1}+\boldsymbol{u}_{2}+\cdots+\boldsymbol{u}_{n}=\left(d n_{0}, d n_{1}, \cdots, d n_{p-1}\right)$, with $d n_{1}+d n_{2}+\cdots+d n_{p-1}=d m$. Therefore, $\boldsymbol{u}_{i}=\boldsymbol{w}_{1}$ for all $1 \leqslant i \leqslant n$, except for at most $d m / 2$ of them, and we have

$$
\begin{aligned}
& \left|\left\{\left(\boldsymbol{u}_{1}, \boldsymbol{u}_{2} \cdots, \boldsymbol{u}_{n}\right) \in \mathcal{U}_{d, p}^{n}: \boldsymbol{u}_{1}+\boldsymbol{u}_{2}+\cdots+\boldsymbol{u}_{n}=\left(d n_{0}, d n_{1}, \cdots, d n_{p-1}\right)\right\}\right| \\
\leqslant & \frac{(d m) !}{\left(d n_{1}\right) !\left(d n_{2}\right) ! \cdots\left(d n_{p-1}\right) !} \sum_{k=1}^{d m / 2} n^{k}\left(\begin{array}{c}
d m-k-1 \\
k-1
\end{array}\right) \leqslant \mathrm{O}(1) \frac{(d m) !}{\left(d n_{1}\right) !\left(d n_{2}\right) ! \cdots\left(d n_{p-1}\right) !} n^{d m / 2} .
\end{aligned}
$$

Putting (3.30) and (3.31) together, the total contribution of terms in (3.14) satisfying $\left|n_{0} / n-1\right| \leqslant \mathfrak{b} p \ln n / n$ is bounded by

$$
\begin{gathered}
\sum_{m=2} \sum_{n_{1}+n_{2}+\cdots+n_{p-1}=m} \frac{e^{\mathrm{O} p \ln n}(d m) !}{n_{1} ! n_{2} ! \cdots n_{p-1} !} \frac{1}{n^{(d / 2-1) m}} \\
\leqslant \\
\sum_{m=2}^{\mathfrak{b} p \ln n} \frac{e^{\mathrm{O}(m)}(d m) ! p^{m}}{m ! n^{(d / 2-1) m}} \leqslant \sum_{m=2}^{\mathfrak{b} p \ln n}\left(\frac{\mathrm{O}(1) p m^{d}}{m n^{d / 2-1}}\right)^{m} \leqslant \frac{\mathrm{O}(1) p^{2}}{n^{d-2}} .
\end{gathered}
$$

provided that $p \ll n^{(d-2) / 2 d}$.

For the last class, the total number of such $p$-tuples is $e^{\mathrm{O}(p \ln n)}$, and thanks to Proposition 3.3, each term is exponentially small, i.e. $e^{-c(\delta) n / p}$. Therefore the total contribution is

$$
\exp \{-c(\delta) n / p+\mathrm{O}(p \ln n)\}=\frac{\mathrm{o}(1)}{n^{(d-2)}} .
$$

The claim (3.14) follows from combining the discussion of all four cases, (3.26), (3.28), (3.32) and (3.33). 


\section{Proof of Theorem 1.1 for random undirected $d$-regular graphs}

Thanks to Proposition 2.2, we can rewrite the lefthand side of (1.2) as

$$
\begin{aligned}
&\left.\sum_{\boldsymbol{v} \in \mathbb{F}_{p}^{n} \backslash \mathbf{0}}\left|\left\{\mathcal{G} \in \mathrm{G}_{n, d}: A(\mathcal{G}) \boldsymbol{v}=\mathbf{0}\right\}\right|=\sum_{\substack{n_{0}, n_{1}, \cdots, n_{p-1} \in \mathbb{Z}_{\geqslant 0}, n_{0}<n \\
n_{0}+n_{1}+\cdots+n_{p-1}=n}} \sum_{\substack{\boldsymbol{v} \in \mathcal{S}\left(n_{0}, n_{1}, \cdots, n_{p-1}\right) \\
n_{0}, n_{1}, \cdots, n_{p-1} \in \mathbb{Z}_{\geqslant 0}, n_{0}<n \\
n_{0}+n_{1}+\cdots+n_{p-1}=n}}\left(\begin{array}{c}
n \\
n_{0}, n_{1}, \cdots, n_{p-1}
\end{array}\right) \sum_{\substack{M \in \mathcal{M}\left(n_{0}, n_{1}, \cdots, n_{p-1}\right) \\
0 \leqslant i<j \leqslant p-1}} \prod_{i j} ! \prod_{i=0}^{p-1} \frac{m_{i i} !}{2^{m_{i i} / 2}\left(m_{i i} / 2\right) !}: A(\mathcal{G}) \boldsymbol{v}=\mathbf{0}\right\} \mid \\
& \times p^{(d-1) n} \prod_{i=0}^{p-1} \mathbb{P}\left(X_{1}+X_{2}+\cdots+X_{n_{i}}=\left(m_{i 0}, m_{i 1}, \cdots, m_{i p-1}\right)\right) \\
&= \sum_{M \in \mathcal{M}}\left(\begin{array}{l}
n \\
\times
\end{array}\right. \\
& \times p^{(d-1) n} \prod_{i=0}^{p-1} \mathbb{P}\left(X_{1}+X_{2}+\cdots+X_{n_{i}}=\left(m_{i 0}, m_{i 1}, \cdots, m_{i p-1}\right)\right), \\
& n_{0}(M), n_{1}(M), \cdots, n_{p-1}(M)
\end{aligned}
$$

where for any $M \in \mathcal{M}, n_{i}(M):=\sum_{j=0}^{p-1} m_{i j} / d$ for $i=0,1, \cdots, p-1$. Therefore Theorem 1.1 is equivalent to the following estiamte

$$
\begin{aligned}
& \frac{2^{n d / 2}(n d / 2) !}{(n d) !} \sum_{M \in \mathcal{M}}\left(\begin{array}{c}
n \\
n_{0}(M), n_{1}(M), \cdots, n_{p-1}(M)
\end{array}\right) \prod_{0 \leqslant i<j \leqslant p-1} m_{i j} ! \prod_{i=0}^{p-1} \frac{m_{i i} !}{2^{m_{i i} / 2}\left(m_{i i} / 2\right) !} \\
& \times p^{(d-1) n} \prod_{i=0}^{p-1} \mathbb{P}\left(X_{1}+X_{2}+\cdots+X_{n_{i}}=\left(m_{i 0}, m_{i 1}, \cdots, m_{i p-1}\right)\right)=1+\mathrm{o}(1) .
\end{aligned}
$$

To prove (4.1), we fix a large number $\mathfrak{b}>0$, and decompose those $p \times p$ symmetric matrices $M=$ $\left[m_{i j}\right]_{0 \leqslant i, j \leqslant p-1} \in \mathcal{M}$ into two classes:

1. (Equidistributed) $\mathcal{E}$ is the set of $p \times p$ symmetric matrices $M=\left[m_{i j}\right]_{0 \leqslant i, j \leqslant p-1} \in \mathcal{M}$, such that $\sum_{i, j=0}^{p-1}\left(m_{i j} /(d n)-1 / p^{2}\right)^{2} \leqslant \mathfrak{b} \ln n / n$.

2. (Non-equidistributed) $\mathcal{N}$ is the set of $p \times p$ symmetric matrices $M=\left[m_{i j}\right]_{0 \leqslant i, j \leqslant p-1} \in \mathcal{M}$ which are not equidistributed.

In Section 4.1, we estimate the sum of terms in (4.1) corresponding to equidistributed $p \times p$ symmetric matrices using a local central limit theorem. In Section 4.2, we show that the sum of terms in (4.1) corresponding to non-equidistributed $p \times p$ symmtric matrices is small, via a large deviation estimate. Theorem 1.1 for random directed $d$-regular graphs follows from combining Proposition 4.1 and Proposition 4.2.

\subsection{Local central limit theorem estimate}

In this section, we estimate the sum of terms in (4.1) corresponding to equidistributed $p \times p$ symmetric matrices $M=\left[m_{i j}\right]_{0 \leqslant i, j \leqslant p-1} \in \mathcal{M}$ using a local central limit theorem. 
Proposition 4.1. Let $d \geqslant 3$ be a fixed integer, and a prime number $p$ such that $\operatorname{gcd}(p, d)=1$ and $p \ll n^{1 / 8}$. Then for $n$ sufficiently large

$$
\sum_{M \in \mathcal{E}} \sum_{\boldsymbol{v} \in \mathcal{S}\left(n_{0}(M), n_{1}(M), \cdots, n_{p-1}(M)\right)}\left|\left\{\mathcal{G} \in \mathrm{G}_{n, d}: A(\mathcal{G}) \boldsymbol{v}=\mathbf{0}\right\}\right|=\left(1+\mathbf{1}(p=2)+\mathrm{O}\left(\frac{p^{4}(\ln n)^{3 / 2}}{\sqrt{n}}\right)\right)\left|\mathrm{G}_{n, d}\right| .
$$

Proof. Thanks to Proposition 2.2, we have

$$
\begin{aligned}
& \frac{1}{\left|\mathrm{G}_{n, d}\right|} \sum_{M \in \mathcal{E}} \sum_{\boldsymbol{v} \in \mathcal{S}\left(n_{0}(M), n_{1}(M), \cdots, n_{p-1}(M)\right)}\left|\left\{\mathcal{G} \in \mathrm{G}_{n, d}: A(\mathcal{G}) \boldsymbol{v}=\mathbf{0}\right\}\right| \\
= & \frac{2^{n d / 2}(n d / 2) !}{(n d) !} \sum_{M \in \mathcal{E}}\left(\begin{array}{c}
n \\
n_{0}(M), n_{1}(M), \cdots, n_{p-1}(M)
\end{array}\right) \prod_{0 \leqslant i<j \leqslant p-1} m_{i j} ! \prod_{i=0}^{p-1} \frac{m_{i i} !}{2^{m_{i i} / 2}\left(m_{i i} / 2\right) !} \\
& \times p^{(d-1) n} \prod_{i=0}^{p-1} \mathbb{P}\left(X_{1}+X_{2}+\cdots+X_{n_{i}}=\left(m_{i 0}, m_{i 1}, \cdots, m_{i p-1}\right)\right),
\end{aligned}
$$

where $X_{1}, X_{2}, \cdots, X_{n_{i}}$ are independent copies of $X$, which is uniform distributed over $\mathcal{U}_{d, p}$ as defined in (2.1). In the rest of the proof, we simply write $n_{i}(M)$ as $n_{i}$ for $0 \leqslant i \leqslant p-1$.

For an equidistributed $p \times p$ symmetric matrix $M=\left[m_{i j}\right]_{0 \leqslant i, j \leqslant p-1}$, we denote $\mathfrak{m}_{i j}=m_{i j} /(d n)$ for $i, j=0,1, \cdots, p-1$, and $\mathfrak{n}_{i}=n_{i} / n$ for $i=0,1, \cdots, p-1$. Then we have $\mathfrak{n}_{i}=\sum_{j=0}^{p-1} \mathfrak{m}_{i j}$ for $i=0,1, \cdots, p-1$. Moreover, by our definition of equidistributed, $\sum_{i, j=0}^{p-1}\left(\mathfrak{m}_{i j}-1 / p^{2}\right)^{2} \leqslant \mathfrak{b} \ln n / n$, and by the AM-GM inequality $\sum_{j=0}^{p-1}\left(\mathfrak{n}_{j}-1 / p\right)^{2} \leqslant \mathfrak{b} p \ln n / n$. We estimate the first factor on the righthand side of (4.3) using Stirling's formula,

$$
\begin{aligned}
& \frac{2^{n d / 2}(n d / 2) !}{(n d) !} \sum_{M \in \mathcal{E}}\left(\begin{array}{c}
n \\
n_{0}, n_{1}, \cdots, n_{p-1}
\end{array}\right) \prod_{0 \leqslant i<j \leqslant p-1} m_{i j} ! \prod_{i=0}^{p-1} \frac{m_{i i} !}{2^{m_{i i} / 2}\left(m_{i i} / 2\right) !} p^{(d-1) n} \\
= & \left(1+\mathrm{O}\left(\frac{p^{4}}{n}\right)\right) 2^{p / 2} \sqrt{\pi n} \frac{\prod_{0 \leqslant i<j \leqslant p-1} \sqrt{2 \pi m_{i j}}}{\prod_{i=0}^{p-1} \sqrt{2 \pi n_{i}}} e^{\frac{d n}{2}} \sum_{i, j=0}^{p-1} \mathfrak{m}_{i j} \ln \mathfrak{m}_{i j}-n \sum_{j=0}^{p-1} \mathfrak{n}_{j} \ln \mathfrak{n}_{j} p^{(d-1) n} \\
= & \left(1+\mathrm{O}\left(\frac{p^{3}(\ln n)^{3 / 2}}{\sqrt{n}}\right)\right) 2^{p / 2} \sqrt{\pi n} \frac{\prod_{0 \leqslant i<j \leqslant p-1} \sqrt{2 \pi d n / p^{2}}}{\prod_{i=0}^{p-1} \sqrt{2 \pi n / p}} e^{\frac{d n p^{2}}{4} \sum_{i, j=0}^{p-1}\left(\mathfrak{m}_{i j}-\frac{1}{p^{2}}\right)^{2}-\frac{n p}{2} \sum_{j=0}^{p-1}\left(\mathfrak{n}_{j}-\frac{1}{p}\right)^{2}} .
\end{aligned}
$$

In the following, we estimate the second factor on the righthand side of (4.3), i.e. $\mathbb{P}\left(S_{n_{i}}=\left(m_{i 0}, m_{i 1}, \cdots, m_{i p-1}\right)\right)$, where $S_{n_{i}}=X_{1}+X_{2}+\cdots+X_{n_{i}}$. We recall that $X_{1}, X_{2}, \cdots, X_{n_{i}}$ are independent copies of $X$, which is uniformly distributed over the multiset $\mathcal{U}_{d, p}$ as defined in (2.1). We use the notation $\boldsymbol{m}_{i}=\left(m_{i 0}, m_{i 1}, m_{i 2}, \cdots, m_{i p-1}\right)$. By inverse Fourier formula

$$
\begin{aligned}
\mathbb{P}\left(S_{n_{i}}=\boldsymbol{m}_{i}\right) & =\frac{1}{(2 \pi)^{p}} \int_{2 \pi \mathbb{R}^{p} / \mathbb{Z}^{p}} \phi_{X}^{n_{i}}(\boldsymbol{t}) e^{-\mathrm{i}\left\langle\boldsymbol{t}, \boldsymbol{m}_{i}\right\rangle} \mathrm{d} \boldsymbol{t} \\
& =\frac{1}{(2 \pi)^{p}} \int_{2 \pi \mathbb{R}^{p} / \mathbb{Z}^{p}} \phi_{X-\boldsymbol{\mu}}^{n_{i}}(\boldsymbol{t}) e^{-\mathrm{i}\left\langle\boldsymbol{t}, \boldsymbol{m}_{i}-n_{i} \boldsymbol{\mu}\right\rangle} \mathrm{d} \boldsymbol{t},
\end{aligned}
$$


where $\phi_{X}(\boldsymbol{t})$ and $\phi_{X-\boldsymbol{\mu}}(\boldsymbol{t})$ are the characteristic functions of $X$ and $X-\boldsymbol{\mu}$ respectively. We recall the domains $B_{j}(\delta)$ for $j=0,1,2, \cdots, p-1$ from (2.15). Thanks to Proposition 2.3, the characteristic function $\left|\phi_{X-\boldsymbol{\mu}}^{n}(\boldsymbol{t})\right|$ is exponentially small outside those sets $B_{j}(\delta)$.

$$
\begin{aligned}
\mathbb{P}\left(S_{n_{i}}=\boldsymbol{m}_{i}\right) & =\frac{1}{(2 \pi)^{p}} \sum_{j=0}^{p-1} \int_{2 \pi B_{j}(\delta)} \phi_{X-\boldsymbol{\mu}}^{n_{i}}(\boldsymbol{t}) e^{-\mathrm{i}\left\langle\boldsymbol{t}, \boldsymbol{m}_{i}-n_{i} \boldsymbol{\mu}\right\rangle} \mathrm{d} \boldsymbol{t}+e^{-c(\delta) n_{i} / p^{3}} \\
& =\frac{p}{(2 \pi)^{p}} \int_{2 \pi B_{0}(\delta)} \phi_{X-\boldsymbol{\mu}}^{n_{i}}(\boldsymbol{t}) e^{-\mathrm{i}\left\langle\boldsymbol{t}, \boldsymbol{m}_{i}-n_{i} \boldsymbol{\mu}\right\rangle} \mathrm{d} \boldsymbol{t}+e^{-c(\delta) n_{i} / p^{3}},
\end{aligned}
$$

where we used the fact that $\sum_{j=0}^{p-1} j m_{i j} \equiv 0(\bmod p)$, and the integrand is translation invariant by vectors $2 \pi(0,1 / p, 2 / p, \cdots, p-1 / p) \mathbb{Z}$. For any $\boldsymbol{t} \in B_{0}(\delta)$, by definition there exists $\boldsymbol{x} \in \mathbb{R}^{p-1}$ with $\|\boldsymbol{x}\|_{2}^{2} \leqslant \delta$ and $y \in[0,2 \sqrt{p} \pi]$, such that $\boldsymbol{t}=Q(\boldsymbol{x}, y)=O \boldsymbol{x}+(y / \sqrt{p}) \mathbf{1}$. By a change of variable, we can rewrite (4.5) as

$$
\begin{aligned}
& \frac{p}{(2 \pi)^{p}} \int_{2 \pi B_{0}(\delta)} \phi_{X-\boldsymbol{\mu}}^{n_{i}}(\boldsymbol{t}) e^{-\mathrm{i}\left\langle\boldsymbol{t}, \boldsymbol{m}_{i}-n_{i} \boldsymbol{\mu}\right\rangle} \mathrm{d} \boldsymbol{t} \\
= & \frac{p}{(2 \pi)^{p}} \int_{\left\{\boldsymbol{x} \in \mathbb{R}^{p-1}:\|\boldsymbol{x}\|_{2}^{2} \leqslant \delta\right\} \times[0,2 \sqrt{p} \pi]} \phi_{X-\boldsymbol{\mu}}^{n_{i}}(Q(\boldsymbol{x}, y)) e^{-\mathrm{i}\left\langle Q(\boldsymbol{x}, y), \boldsymbol{m}_{i}-n_{i} \boldsymbol{\mu}\right\rangle} \mathrm{d} \boldsymbol{x} \mathrm{d} y \\
= & \frac{p^{3 / 2}}{(2 \pi)^{p-1}} \int_{\left\{\boldsymbol{x} \in \mathbb{R}^{p-1}:\|\boldsymbol{x}\|_{2}^{2} \leqslant \delta\right\}} \phi_{X-\boldsymbol{\mu}}^{n_{i}}(O \boldsymbol{x}) e^{-\mathrm{i}\left\langle O \boldsymbol{x}, \boldsymbol{m}_{i}-n_{i} \boldsymbol{\mu}\right\rangle} \mathrm{d} \boldsymbol{x},
\end{aligned}
$$

where we used that $\langle\mathbf{1}, X-\boldsymbol{\mu}\rangle=0$ and $\left\langle\mathbf{1}, \boldsymbol{m}_{i}-n_{i} \boldsymbol{\mu}\right\rangle=0$. We recall the estimate of the characteristic function from (3.7),

$$
\phi_{X-\mu}(O \boldsymbol{x})=1-\frac{d}{2 p}\|\boldsymbol{x}\|_{2}^{2}+\mathrm{O}\left(\frac{\|\boldsymbol{x}\|_{2}^{3}}{p}\right)
$$

Fix a large constant $\mathfrak{c}$, which will be chosen later. For $\mathfrak{c} p^{4} \ln n / n \leqslant\|\boldsymbol{x}\|_{2}^{2} \leqslant \delta$, we have

$$
\left|\phi_{X-\boldsymbol{\mu}}(O \boldsymbol{x})\right|^{n_{i}} \leqslant \exp \left\{-\left(\frac{\mathfrak{c} d}{2}+\mathrm{o}(1)\right) p^{2} \ln n\right\}
$$

which turns out to be negligible provided $\mathfrak{c}$ is large enough. In the following we will restrict the integral (4.6) on the domain $\left\{\boldsymbol{x} \in \mathbb{R}^{p-1}:\|\boldsymbol{x}\|_{2}^{2} \leqslant \mathfrak{c} p^{4} \ln n / n\right\}$. From (4.7), on the domain $\left\{\boldsymbol{x} \in \mathbb{R}^{p-1}:\|\boldsymbol{x}\|_{2}^{2} \leqslant \mathfrak{c} p^{4} \ln n / n\right\}$, we have

$$
\phi_{X-\boldsymbol{\mu}}^{n_{i}}(O \boldsymbol{x})=\left(1+\mathrm{O}\left(\frac{p^{4}(\ln n)^{3 / 2}}{n^{1 / 2}}\right)\right) e^{-\frac{d n_{i}}{2 p}\|\boldsymbol{x}\|_{2}^{2}}
$$


and

$$
\begin{aligned}
& \frac{p^{3 / 2}}{(2 \pi)^{p-1}} \int_{\left\{\boldsymbol{x} \in \mathbb{R}^{p-1}:\|\boldsymbol{x}\|_{2}^{2} \leqslant \mathfrak{c} p^{4} \ln n / n\right\}} \phi_{X-\boldsymbol{\mu}}^{n_{i}}(O \boldsymbol{x}) e^{-\mathrm{i}\left\langle O \boldsymbol{x}, \boldsymbol{m}_{i}-n_{i} \boldsymbol{\mu}\right\rangle} \mathrm{d} \boldsymbol{x} \\
= & \left(1+\mathrm{O}\left(\frac{p^{4}(\ln n)^{3 / 2}}{n^{1 / 2}}\right)\right) \frac{p^{3 / 2}}{(2 \pi)^{p-1}} \int_{\left\{\boldsymbol{x} \in \mathbb{R}^{p-1}:\|\boldsymbol{x}\|_{2}^{2} \leqslant \mathfrak{c} p^{4} \ln n / n\right\}} e^{-\frac{d n_{i}}{2 p}\|\boldsymbol{x}\|_{2}^{2}} e^{-\mathrm{i}\left\langle\boldsymbol{x}, O^{t}\left(\boldsymbol{m}_{i}-n_{i} \boldsymbol{\mu}\right)\right\rangle} \mathrm{d} \boldsymbol{x} \\
= & \left(1+\mathrm{O}\left(\frac{p^{4}(\ln n)^{3 / 2}}{n^{1 / 2}}\right)\right) \frac{p^{3 / 2}}{(2 \pi)^{p-1}} \int_{\mathbb{R}^{p-1}} e^{-\frac{d n_{i}}{2 p}\|\boldsymbol{x}\|_{2}^{2}} e^{-\mathrm{i}\left\langle\boldsymbol{x}, O^{t}\left(\boldsymbol{m}_{i}-n_{i} \boldsymbol{\mu}\right)\right\rangle} \mathrm{d} \boldsymbol{x}+\mathrm{O}\left(e^{-\left(\frac{\mathrm{c} d}{2}+\mathrm{o}(1)\right) p^{2} \ln n}\right) \\
= & \left(1+\mathrm{O}\left(\frac{p^{4}(\ln n)^{3 / 2}}{n^{1 / 2}}\right)\right) \frac{p^{3 / 2}}{(2 \pi)^{p-1}} \int_{\mathbb{R}^{p-1}} e^{-\frac{d n_{i}}{2 p}\|\boldsymbol{x}\|_{2}^{2}} e^{-\mathrm{i}\left\langle\boldsymbol{x}, O^{t}\left(\boldsymbol{m}_{i}-n_{i} \boldsymbol{\mu}\right)\right\rangle} \mathrm{d} \boldsymbol{x}+\mathrm{O}\left(e^{-\left(\frac{\mathrm{c} d}{2}+\mathrm{o}(1)\right) p^{2} \ln n}\right) \\
= & \left(1+\mathrm{O}\left(\frac{p^{4}(\ln n)^{3 / 2}}{n^{1 / 2}}\right)\right) p^{3 / 2}\left(\frac{p}{2 \pi d n_{i}}\right)^{(p-1) / 2} e^{-\frac{n_{i} p d}{2}\left\|O^{t}\left(\frac{m_{i}}{d n_{i}}-\frac{\mu}{d}\right)\right\|_{2}^{2}}+\mathrm{O}\left(e^{-\left(\frac{c d}{2}+\mathrm{o}(1)\right) p^{2} \ln n}\right) \\
= & \left(1+\mathrm{O}\left(\frac{p^{4}(\ln n)^{3 / 2}}{n^{1 / 2}}\right)\right) p^{3 / 2}\left(\frac{p^{2}}{2 \pi d n}\right)^{(p-1) / 2} e^{-\frac{n d}{2} \sum_{j=0}^{p-1}\left(\frac{\mathfrak{m}_{i j}}{\boldsymbol{n}_{i}}-\frac{1}{p}\right)^{2}}+\mathrm{O}\left(e^{-\left(\frac{\mathrm{c} d}{2}+\mathrm{o}(1)\right) p^{2} \ln n}\right) .
\end{aligned}
$$

By our definition that $M$ is equidistributed, $\sum_{i, j=0}^{p-1}\left(\mathfrak{m}_{i j}-1 / p^{2}\right)^{2} \leqslant \mathfrak{b} \ln n / n$, and by the AM-GM inequlity $\sum_{j=0}^{p-1}\left(\mathfrak{n}_{j}-1 / p\right)^{2} \leqslant \mathfrak{b} p \ln n / n$. We can rewrite the exponent in (4.9) as

$$
-\frac{n d}{2} \sum_{j=0}^{p-1}\left(\frac{\mathfrak{m}_{i j}}{\mathfrak{n}_{i}}-\frac{1}{p}\right)^{2}=-\frac{d n p}{2}\left(p \sum_{j=0}^{p-1}\left(\mathfrak{m}_{i j}-\frac{1}{p^{2}}\right)^{2}-\left(\mathfrak{n}_{i}-\frac{1}{p}\right)^{2}\right)+\mathrm{O}\left(\frac{p^{3}(\ln n)^{3 / 2}}{n^{1 / 2}}\right) .
$$

It follows by combining (4.9) and (4.10),

$$
\begin{aligned}
& \prod_{i=0}^{p-1} \mathbb{P}\left(X_{1}+X_{2}+\cdots+X_{n_{i}}=\left(m_{i 0}, m_{i 1}, \cdots, m_{i p-1}\right)\right)=\mathrm{O}\left(e^{-\left(\frac{c d}{2}+\mathrm{o}(1)\right) p^{2} \ln n}\right) \\
& \left.+\left(1+\mathrm{O}\left(\frac{p^{4}(\ln n)^{3 / 2}}{n^{1 / 2}}\right)\right) p^{3 p / 2}\left(\frac{p^{2}}{2 \pi d n}\right)^{\left(p^{2}-p\right) / 2} e^{-\frac{d n p}{2}\left(p \sum_{i, j=0}^{p-1}\left(\mathfrak{m}_{i j}-\frac{1}{p^{2}}\right)^{2}-\sum_{i=0}^{p-1}\left(\mathfrak{n}_{i}-\frac{1}{p}\right)^{2}\right.}\right) .
\end{aligned}
$$

The exponents in (4.4) and (4.11) cancel

$$
\begin{aligned}
& \frac{d n p^{2}}{4} \sum_{i, j=0}^{p-1}\left(\mathfrak{m}_{i j}-\frac{1}{p^{2}}\right)^{2}-\frac{n p}{2} \sum_{j=0}^{p-1}\left(\mathfrak{n}_{j}-\frac{1}{p}\right)^{2}-\frac{d n p}{2}\left(p \sum_{i, j=0}^{p-1}\left(\mathfrak{m}_{i j}-\frac{1}{p^{2}}\right)^{2}-\sum_{i=0}^{p-1}\left(\mathfrak{n}_{i}-\frac{1}{p}\right)^{2}\right) \\
= & -\frac{d n p^{2}}{4} \sum_{i, j=0}^{p-1}\left(\mathfrak{m}_{i j}-\frac{1}{p^{2}}\right)^{2}+\frac{(d-1) n p}{2} \sum_{j=0}^{p-1}\left(\mathfrak{n}_{j}-\frac{1}{p}\right)^{2} .
\end{aligned}
$$

Therefore, by combining the estimates (4.4), (4.11) and (4.12), we conclude that for any equidistributed $p \times p$ symmetric matrices $M=\left[m_{i j}\right]_{0 \leqslant i, j \leqslant p-1} \in \mathcal{M}$,

$$
\begin{aligned}
& \frac{1}{\left|\mathrm{G}_{n, d}\right|} \sum_{\boldsymbol{v} \in \mathcal{S}\left(n_{0}(M), n_{1}(M), \cdots, n_{p-1}(M)\right)}\left|\left\{\mathcal{G} \in \mathrm{G}_{n, d}: A(\mathcal{G}) \boldsymbol{v}=\mathbf{0}\right\}\right|=\mathrm{O}\left(e^{-\left(\frac{\mathfrak{c} d}{2}-\frac{\mathfrak{b} d}{4}+\mathrm{o}(1)\right) p^{2} \ln n}\right) \\
& +\left(1+\mathrm{O}\left(\frac{p^{4}(\ln n)^{3 / 2}}{n^{1 / 2}}\right)\right) \frac{2^{(p-1) / 2} p^{\left(p^{2}+3 p\right) / 2}}{d^{\left(p^{2}-p\right) / 4}(2 \pi n)^{\left(p^{2}+p-2\right) / 4}} e^{-\frac{d n p^{2}}{4} \sum_{i, j=0}^{p-1}\left(\mathfrak{m}_{i j}-\frac{1}{p^{2}}\right)^{2}+\frac{(d-1) n p}{2} \sum_{j=0}^{p-1}\left(\mathfrak{n}_{j}-\frac{1}{p}\right)^{2}} .
\end{aligned}
$$


For the first term on the righthand side of (4.13), we notice that the total number of $p \times p$ symmetric matrices in $\mathcal{E}$ is bounded by $e^{p^{2} \ln n}$,

$$
\sum_{M \in \mathcal{E}} \mathrm{O}\left(e^{-\left(\frac{\mathfrak{c} d}{2}-\frac{\mathfrak{b} d}{4}+\mathrm{o}(1)\right) p^{2} \ln n}\right) \leqslant \mathrm{O}\left(e^{-\left(\frac{\mathfrak{c} d}{2}-\frac{\mathfrak{b} d}{4}-1+\mathrm{o}(1)\right) p^{2} \ln n}\right),
$$

which is negligible provided $\mathfrak{c}$ is large enough.

For the second term on the righthand side of (4.13), we denote $\tilde{\mathcal{E}}$ the set of $p \times p$ symmetric matrices $M=\left[m_{i j}\right]_{0 \leqslant i, j \leqslant p}$ such that

1. $m_{i j}=m_{j i} \in \mathbb{Z}_{\geqslant 0}$ for $0 \leqslant i, j \leqslant p-1$ and $2 \mid m_{i i}$ for $0 \leqslant i \leqslant p-1$.

2. $\sum_{i, j=0}^{p-1} m_{i j}=d n$, and $\sum_{i, j=0}^{p-1}\left(m_{i j} /(d n)-1 / p^{2}\right)^{2} \leqslant \mathfrak{b} \ln n / n$.

The set $\mathcal{E}$ is a subset of $\tilde{\mathcal{E}}$ with the extra constraints: $\sum_{j=0}^{p-1} m_{i j} \equiv 0(\bmod d)$, and $\sum_{j=0}^{p-1} j m_{i j} \equiv 0(\bmod p)$ for $i=0,1, \cdots, p-1$. In the following we prove that the sum over the set $\mathcal{E}$ in (4.3) can be replaced by the sum over the set $\tilde{\mathcal{E}}$ with a negligible error. We concentrate on the case $p$ is odd, and remark the modification for $p=2$ case later.

By our assumption $\operatorname{gcd}(p, d)=1$, for any vectors $\boldsymbol{r}=\left(r_{0}, r_{1}, \cdots, r_{p-1}\right) \in\{0,1, \cdots, d-1\}^{p}$ with $r_{0}+$ $r_{1}+\cdots+r_{p-1} \equiv 0(\bmod d)$, and $s=\left(s_{0}, s_{1}, \cdots, s_{p-1}\right) \in\{0,1, \cdots, p-1\}^{p}$, there exists a $p \times p$ matrix $\left[\Delta m_{i j}^{(\boldsymbol{r}, \boldsymbol{s})}\right]_{0 \leqslant i, j \leqslant p-1}$ with entries size $\mathrm{O}(p)$ (not unique), such that

1. $\Delta m_{i j}^{(\boldsymbol{r}, \boldsymbol{s})}=\Delta m_{j i}^{(\boldsymbol{r}, \boldsymbol{s})} \in \mathbb{Z}_{\geqslant 0}$ for $0 \leqslant i, j \leqslant p-1$ and $2 \mid \Delta m_{i i}^{(\boldsymbol{r}, \boldsymbol{s})}$ for $0 \leqslant i \leqslant p-1$.

2. $\sum_{i, j=0}^{p-1} \Delta m_{i j}^{(\boldsymbol{r}, \boldsymbol{s})}=0$.

3. $\sum_{j=0}^{p-1} \Delta m_{i j}^{(\boldsymbol{r}, \boldsymbol{s})} \equiv r_{i}(\bmod d), \sum_{j=0}^{p-1} j \Delta m_{i j}^{(\boldsymbol{r}, \boldsymbol{s})} \equiv s_{i}(\bmod p)$, for $i=0,1, \cdots, p-1$.

For the term on the righthand side of (4.13) corresponding to $M=\left[m_{i j}\right]_{0 \leqslant i, j \leqslant p-1} \in \mathcal{E}$, we can rewrite it as an average of terms corresponding to $M^{(\boldsymbol{r}, \boldsymbol{s})}=\left[m_{i j}^{(\boldsymbol{r}, \boldsymbol{s})}:=m_{i j}+\Delta m_{i j}^{(\boldsymbol{r}, \boldsymbol{s})}\right]_{0 \leqslant i, j \leqslant p-1} \in \tilde{\mathcal{E}}$, for $\boldsymbol{r}=$ $\left(r_{0}, r_{1}, \cdots, r_{p-1}\right) \in\{0,1, \cdots, d-1\}^{p}$ with $r_{0}+r_{1}+\cdots+r_{p-1} \equiv 0(\bmod d)$, and $s=\left(s_{0}, s_{1}, \cdots, s_{p-1}\right) \in$ $\{0,1, \cdots, p-1\}^{p}$,

$$
\begin{aligned}
& d^{p-1} p^{p}\left(1+\mathrm{O}\left(\frac{p^{4}(\ln n)^{1 / 2}}{n^{1 / 2}}\right)\right) e^{-\frac{d n p^{2}}{4} \sum_{i, j=0}^{p-1}\left(\frac{m_{i j}}{d n}-\frac{1}{p^{2}}\right)^{2}+\frac{(d-1) n p}{2} \sum_{j=0}^{p-1}\left(\frac{n_{i}}{n}-\frac{1}{p}\right)^{2}} \\
= & \sum_{\substack{r \in\{0,1, \cdots, d-1\}^{p} \\
\sum_{i=0}^{p-1} r_{i} \equiv 0(\bmod d)}} \sum_{s \in\{0,1, \cdots, p-1\}^{p}} e^{-\frac{d n p^{2}}{4} \sum_{i, j=0}^{p-1}\left(\frac{m_{i j}^{(r, s)}}{d n}-\frac{1}{p^{2}}\right)^{2}+\frac{(d-1) n p}{2} \sum_{j=0}^{p-1}\left(\frac{n_{i}^{(r, s)}}{n}-\frac{1}{p}\right)^{2},},
\end{aligned}
$$

where $n_{i}^{(\boldsymbol{r}, \boldsymbol{s})}=\sum_{j=0}^{p-1} m_{i j}^{(\boldsymbol{r}, \boldsymbol{s})}$, for $i=0,1, \cdots, p-1$. We sum (4.15) over all the $p \times p$ symmetric matrices $M=\left[m_{i j}\right]_{0 \leqslant i, j \leqslant p-1} \in \mathcal{E}$, and get

$$
\begin{aligned}
& d^{p-1} p^{p} \sum_{M \in \mathcal{E}} e^{-\frac{d n p^{2}}{4} \sum_{i, j=0}^{p-1}\left(\mathfrak{m}_{i j}-\frac{1}{p^{2}}\right)^{2}+\frac{(d-1) n p}{2} \sum_{j=0}^{p-1}\left(\mathfrak{n}_{j}-\frac{1}{p}\right)^{2}} \\
= & \left(1+\mathrm{O}\left(\frac{p^{4}(\ln n)^{1 / 2}}{n^{1 / 2}}\right)\right) \sum_{M \in \tilde{\mathcal{E}}} e^{-\frac{d n p^{2}}{4} \sum_{i, j=0}^{p-1}\left(\mathfrak{m}_{i j}-\frac{1}{p^{2}}\right)^{2}+\frac{(d-1) n p}{2} \sum_{j=0}^{p-1}\left(\mathfrak{n}_{j}-\frac{1}{p}\right)^{2}} .
\end{aligned}
$$


We remark that if $p=2$, we have instead that

$$
\begin{aligned}
& d^{p-1} p^{p-1} \sum_{M \in \mathcal{E}} e^{-\frac{d n p^{2}}{4} \sum_{i, j=0}^{p-1}\left(\mathfrak{m}_{i j}-\frac{1}{p^{2}}\right)^{2}+\frac{(d-1) n p}{2} \sum_{j=0}^{p-1}\left(\mathfrak{n}_{j}-\frac{1}{p}\right)^{2}} \\
= & \left(1+\mathrm{O}\left(\frac{p^{4}(\ln n)^{1 / 2}}{n^{1 / 2}}\right)\right) \sum_{M \in \tilde{\mathcal{E}}} e^{-\frac{d n p^{2}}{4} \sum_{i, j=0}^{p-1}\left(\mathfrak{m}_{i j}-\frac{1}{p^{2}}\right)^{2}+\frac{(d-1) n p}{2} \sum_{j=0}^{p-1}\left(\mathfrak{n}_{j}-\frac{1}{p}\right)^{2}},
\end{aligned}
$$

which differs from (4.16) by a factor of 2. As a consequence, this leads to $\sum_{\boldsymbol{v} \in \mathbb{F}_{p}^{n} \backslash \mathbf{0}} \mid\left\{\mathcal{G} \in \mathrm{G}_{n, d}: A(\mathcal{G}) \boldsymbol{v}=\right.$ $\mathbf{0}\}|=(2+\mathrm{o}(1))| \mathrm{G}_{n, d} \mid$.

In the following we estimate the sum in (4.16). The set of points $\left[m_{i j} / d n-1 / p^{2}\right]_{0 \leqslant i, j \leqslant p-1}$ for $M=$ $\left[m_{i j}\right]_{0 \leqslant i, j \leqslant p-1} \in \tilde{\mathcal{E}}$ is a subset of a lattice in $\operatorname{Sym}_{p}^{0}$, the Hilbert space of $p \times p$ real symmetric matrices with total sum zero, and inner product $\langle A, B\rangle=\operatorname{Tr} A B$. A set of base for this lattice is given by

$$
\left(e_{i j}+e_{j i}-2 e_{00}\right) / d n, \quad 0 \leqslant i<j \leqslant p-1, \quad\left(2 e_{i i}-2 e_{00}\right) / d n, \quad 1 \leqslant i \leqslant p-1 .
$$

The volume of the fundamental domain is $2^{\left(p^{2}+3 p-4\right) / 4} p(d n)^{-\left(p^{2}+p-2\right) / 2}$. By viewing (4.16) as a Riemann sum, we can rewrite it as an integral on the space $\operatorname{Sym}_{p}^{0}$.

$$
\begin{aligned}
& 2^{\left(p^{2}+3 p-4\right) / 4} p(d n)^{-\left(p^{2}+p-2\right) / 2} \sum_{M \in \tilde{\mathcal{E}}} e^{-\frac{d n p^{2}}{4} \sum_{i, j=0}^{p-1}\left(\mathfrak{m}_{i j}-\frac{1}{p^{2}}\right)^{2}+\frac{(d-1) n p}{2} \sum_{j=0}^{p-1}\left(\mathfrak{n}_{j}-\frac{1}{p}\right)^{2}} \\
= & \left(1+\mathrm{O}\left(\frac{p^{4}(\ln n)^{1 / 2}}{n^{1 / 2}}\right)\right) \int_{A \in \operatorname{Sym}_{p}^{0}:\|A\|_{2}^{2} \leqslant \mathfrak{b} \ln n / n} e^{-\frac{d n p^{2}}{4} \operatorname{Tr} A^{2}+\frac{(d-1) n p}{2} \mathbf{1}^{t} A^{2} \mathbf{1}} \mathrm{d} \operatorname{vol}(A) .
\end{aligned}
$$

We can rewrite the exponent as a quadratic form on the space $\operatorname{Sym}_{p}^{0}$,

$$
-d n p^{2} \operatorname{Tr} A^{2} / 4+(d-1) n p \mathbf{1}^{t} A^{2} \mathbf{1} / 2=\langle A, \mathcal{L}(A)\rangle,
$$

where the self-adjoint operator $\mathcal{L}: \operatorname{Sym}_{p}^{0} \mapsto \operatorname{Sym}_{p}^{0}$ is given by $\mathcal{L}(A)=-d n p^{2} A / 4+(d-1) n p A \mathbf{1 1}^{t} / 4+(d-$ 1) $n p \mathbf{1 1}^{t} A / 4$. The self-adjoint operator $\mathcal{L}$ is diagonalized by

1. If $A \in \operatorname{Sym}_{p}^{0}$, with row sums and column sums zero, then $\mathcal{L}(A)=\left(-d n p^{2} / 4\right) A$. The total dimension of such matrices is $p(p-1) / 2$.

2. If $A=\left[a_{i}+a_{j}\right]_{0 \leqslant i, j \leqslant p-1}$ for some vector $\boldsymbol{a}=\left(a_{0}, a_{1}, \cdots, a_{p-1}\right) \in \mathbb{R}^{p}$ with $a_{0}+a_{1}+\cdots+a_{p-1}=0$, then $\mathcal{L}(A)=\left(-n p^{2} / 4\right) A$. The total dimension of such matrices is $p-1$.

From the discussion above, using the eigenvectors of the self-adjoint operator $\mathcal{L}$ as a base, the integral (4.17) decomposes into a product of Gaussian integrals, which can be estimated explicitly.

$$
\begin{aligned}
& \int_{A \in \operatorname{Sym}_{p}^{0}:\|A\|_{2}^{2} \leqslant \mathfrak{b} \ln n / n} e^{-\frac{d n p^{2}}{4} \operatorname{Tr} A^{2}+\frac{(d-1) n p}{2} \mathbf{1}^{t} A^{2} \mathbf{1}} \mathrm{d} \operatorname{vol}(A) \\
= & \int_{A \in \operatorname{Sym}_{p}^{0}} e^{-\frac{d n p^{2}}{4} \operatorname{Tr} A^{2}+\frac{(d-1) n p}{2} \mathbf{1}^{t} A^{2} \mathbf{1}} \mathrm{d} \operatorname{vol}(A)+\mathrm{O}\left(e^{-\left(\frac{\mathfrak{b}}{4}+\mathrm{o}(1)\right) p^{2} \ln n}\right) \\
= & \left(\frac{4 \pi}{d n p^{2}}\right)^{\left(p^{2}-p\right) / 4}\left(\frac{4 \pi}{n p^{2}}\right)^{(p-1) / 2}+\mathrm{O}\left(e^{-\left(\frac{\mathfrak{b}}{4}+\mathrm{o}(1)\right) p^{2} \ln n}\right) .
\end{aligned}
$$


We can estimate the total contribution in (4.2) from the second term on the righthand side of (4.13), by combining the estimates (4.16), (4.17) and (4.18)

$$
\begin{aligned}
& \left(1+\mathrm{O}\left(\frac{p^{4}(\ln n)^{3 / 2}}{n^{1 / 2}}\right)\right) \frac{2^{(p-1) / 2} p^{\left(p^{2}+3 p\right) / 2}}{d^{\left(p^{2}-p\right) / 4}(2 \pi n)^{\left(p^{2}+p-2\right) / 4}} \sum_{M \in \mathcal{E}} e^{-\frac{d n p^{2}}{4} \sum_{i, j=0}^{p-1}\left(\mathfrak{m}_{i j}-\frac{1}{p^{2}}\right)^{2}+\frac{(d-1) n p}{2} \sum_{j=0}^{p-1}\left(\mathfrak{n}_{j}-\frac{1}{p}\right)^{2}} \\
= & \left(1+\mathrm{O}\left(\frac{p^{4}(\ln n)^{3 / 2}}{n^{1 / 2}}\right)\right)+\mathrm{O}\left(e^{-\left(\frac{\mathfrak{b}}{4}+\mathrm{o}(1)\right) p^{2} \ln n+\frac{p^{2}+p-2}{4} \ln \left(n p^{2}\right)}\right)=1+\mathrm{O}\left(\frac{p^{4}(\ln n)^{3 / 2}}{n^{1 / 2}}\right),
\end{aligned}
$$

provided $\mathfrak{b}$ is large enough. Proposition 4.1 follows from combining (4.14) and (4.19).

\subsection{Large deviation estimate}

In this section, we show that the sum of terms in (4.1) corresponding to non-equidistributed $p \times p$ symmetric matrices $M=\left[m_{i j}\right]_{0 \leqslant i, j \leqslant p-1}$ is small.

Proposition 4.2. Let $d \geqslant 3$ be a fixed integer, and a prime number $p$ such that $\operatorname{gcd}(p, d)=1$ and $p \ll$ $n^{(d-2) /(5 d-6)}$. Then for $n$ sufficiently large,

$$
\frac{1}{\left|\mathrm{G}_{n, d}\right|} \sum_{M \in \mathcal{N}} \sum_{\boldsymbol{v} \in \mathcal{S}\left(n_{0}(M), n_{1}(M), \cdots, n_{p-1}(M)\right)}\left|\left\{\mathcal{G} \in \mathrm{G}_{n, d}: A(\mathcal{G}) \boldsymbol{v}=\mathbf{0}\right\}\right| \leqslant \frac{\mathrm{O}\left(p^{2 d}\right)}{n^{(d-2)}} .
$$

Thanks to Proposition 2.2, we have

$$
\begin{aligned}
& \frac{1}{\left|\mathrm{G}_{n, d}\right|} \sum_{M \in \mathcal{N}} \sum_{\boldsymbol{v} \in \mathcal{S}\left(n_{0}(M), n_{1}(M), \cdots, n_{p-1}(M)\right)}\left|\left\{\mathcal{G} \in \mathrm{G}_{n, d}: A(\mathcal{G}) \boldsymbol{v}=\mathbf{0}\right\}\right| \\
= & \frac{2^{n d / 2}(n d / 2) !}{(n d) !} \sum_{M \in \mathcal{N}}\left(\begin{array}{c}
n \\
n_{0}(M), n_{1}(M), \cdots, n_{p-1}(M)
\end{array}\right) \prod_{0 \leqslant i<j \leqslant p-1} m_{i j} ! \prod_{i=0}^{p-1} \frac{m_{i i} !}{2^{m_{i i} / 2}\left(m_{i i} / 2\right) !} \\
& \times p^{(d-1) n} \prod_{i=0}^{p-1} \mathbb{P}\left(X_{1}+X_{2}+\cdots+X_{n_{i}}=\left(m_{i 0}, m_{i 1}, \cdots, m_{i p-1}\right)\right)
\end{aligned}
$$

where $X_{1}, X_{2}, \cdots, X_{n_{i}}$ are independent copies of $X$, which is uniform distributed over $\mathcal{U}_{d, p}$ as defined in (2.1). In the rest of the proof, we simply write $n_{i}(M)$ as $n_{i}$ for $0 \leqslant i \leqslant p-1$. For an non-equidistributed $p \times p$ symmetric matrix $M=\left[m_{i j}\right]_{0 \leqslant i, j \leqslant p-1}$, we denote $\mathfrak{m}_{i j}=m_{i j} /(d n)$ for $i, j=0,1, \cdots, p-1$, and $\mathfrak{n}_{i}=n_{i} / n$ for $i=0,1, \cdots, p-1$. Then we have $\mathfrak{n}_{i}=\sum_{j=0}^{p-1} \mathfrak{m}_{i j}$ for $i=0,1, \cdots, p-1$. Moreover, by our definition of non-equidistributed, $\sum_{i, j=0}^{p-1}\left(\mathfrak{m}_{i j}-1 / p^{2}\right)^{2} \geqslant \mathfrak{b} \ln n / n$. We estimate the first factor on the righthand side of (4.21) using Stirling's formula,

$$
\begin{aligned}
& \frac{2^{n d / 2}(n d / 2) !}{(n d) !}\left(\begin{array}{c}
n \\
n_{0}, n_{1}, \cdots, n_{p-1}
\end{array}\right) \prod_{0 \leqslant i<j \leqslant p-1} m_{i j} ! \prod_{i=0}^{p-1} \frac{m_{i i} !}{2^{m_{i i} / 2}\left(m_{i i} / 2\right) !} \\
\leqslant & e^{\mathrm{O}\left(p^{2} \ln n\right)} \exp \left\{\frac{d n}{2} \sum_{i, j=0}^{p-1} \mathfrak{m}_{i j} \ln \mathfrak{m}_{i j}-n \sum_{j=0}^{p-1} \mathfrak{n}_{j} \ln \mathfrak{n}_{j}\right\} .
\end{aligned}
$$


For the random walk term in (4.21), we have the following large deviation bound

$$
\mathbb{P}\left(X_{1}+X_{2}+\cdots+X_{n_{i}}=\left(m_{i 0}, m_{i 1}, \cdots, m_{i p-1}\right)\right) \leqslant \exp \left\{n \inf _{\boldsymbol{t}_{i} \in \mathbb{R}^{p}} \mathfrak{n}_{i} \log \mathbb{E}\left[e^{\left\langle\boldsymbol{t}_{i}, X\right\rangle}\right]-d\left\langle\boldsymbol{t}_{i}, \mathfrak{m}_{i}\right\rangle\right\},
$$

where $\mathfrak{m}_{i}=\left(\mathfrak{m}_{i 0}, \mathfrak{m}_{i 1}, \cdots, \mathfrak{m}_{i p-1}\right)$. Thus we get that

$$
\begin{aligned}
& \frac{1}{\left|\mathrm{G}_{n, d}\right|} \sum_{M \in \mathcal{N}} \sum_{\boldsymbol{v} \in \mathcal{S}\left(n_{0}(M), n_{1}(M), \cdots, n_{p-1}(M)\right)}\left|\left\{\mathcal{G} \in \mathrm{G}_{n, d}: A(\mathcal{G}) \boldsymbol{v}=\mathbf{0}\right\}\right| \\
\leqslant & \sum_{M \in \mathcal{N}} \sum_{\boldsymbol{v} \in \mathcal{S}\left(n_{0}(M), n_{1}(M), \cdots, n_{p-1}(M)\right)} e^{\mathrm{O}\left(p^{2} \ln n\right)} e^{n I\left(\mathfrak{m}_{0}, \mathfrak{m}_{1}, \cdots, \mathfrak{m}_{p-1}\right)}
\end{aligned}
$$

where the rate function is given by

$$
\begin{aligned}
I\left(\mathfrak{m}_{0}, \mathfrak{m}_{1}, \cdots, \mathfrak{m}_{p-1}\right) & =(d-1) \ln p+\frac{d}{2} \sum_{i, j=0}^{p-1} \mathfrak{m}_{i j} \ln \mathfrak{m}_{i j}-\sum_{j=0}^{p-1} \mathfrak{n}_{j} \ln \mathfrak{n}_{j} \\
& +\sum_{j=0}^{p-1} \inf _{\boldsymbol{t}_{j} \in \mathbb{R}^{p}} \mathfrak{n}_{j} \log \mathbb{E}\left[e^{\left\langle\boldsymbol{t}_{j}, X\right\rangle}\right]-d\left\langle\boldsymbol{t}_{j}, \mathfrak{m}_{j}\right\rangle .
\end{aligned}
$$

Proposition 4.3. Let $d \geqslant 3$ be a fixed integer, and a prime number $p$ such that $\operatorname{gcd}(p, d)=1$. The rate function as defined in (4.23) satisfies: for any small $\delta>0$, there exists a constant $c(\delta)>0$, such that

$$
I\left(\mathfrak{m}_{0}, \mathfrak{m}_{1}, \cdots, \mathfrak{m}_{p-1}\right) \leqslant-\frac{c(\delta)}{p^{2}}
$$

unless $\max _{0 \leqslant i, j \leqslant p-1}\left|\mathfrak{m}_{i j}-1 / p^{2}\right| \leqslant \delta / p^{2}$, or $\mathfrak{m}_{00} \geqslant 1-\delta / p$.

Proof. We take $\boldsymbol{t}_{j}=(d-1) / d\left(\left(\ln \left(\mathfrak{m}_{j 0} / \mathfrak{n}_{j}\right), \ln \left(\mathfrak{m}_{j 1} / \mathfrak{n}_{j}\right), \cdots, \ln \left(\mathfrak{m}_{j p-1} / \mathfrak{n}_{j}\right)\right)+\ln p\right)$ in (4.23), the rate function $I$ is upper bounded by

$$
I\left(\mathfrak{m}_{0}, \mathfrak{m}_{1}, \cdots, \mathfrak{m}_{p-1}\right) \leqslant \frac{d-2}{2} \sum_{i, j=0}^{p-1} \mathfrak{m}_{i j} \ln \frac{\mathfrak{n}_{i} \mathfrak{n}_{j}}{\mathfrak{m}_{i j}}+\sum_{i=0}^{p-1} \mathfrak{n}_{i} \log \sum_{j=1}^{p^{d-1}} \prod_{k=0}^{p-1}\left(\frac{\mathfrak{m}_{i k}}{\mathfrak{n}_{i}}\right)^{\frac{d-1}{d} \boldsymbol{w}_{j}(k)} .
$$

In the following, we prove that there exists a constant $c(\delta)$

$$
\frac{d-2}{2} \sum_{i, j=0}^{p-1} \mathfrak{m}_{i j} \ln \frac{\mathfrak{n}_{i} \mathfrak{n}_{j}}{\mathfrak{m}_{i j}}+\sum_{i=0}^{p-1} \mathfrak{n}_{i} \log \sum_{j=1}^{p^{d-1}} \prod_{k=0}^{p-1}\left(\frac{\mathfrak{m}_{i k}}{\mathfrak{n}_{i}}\right)^{\frac{d-1}{d} \boldsymbol{w}_{j}(k)} \leqslant-\frac{c(\delta)}{p^{2}},
$$

unless $\max _{0 \leqslant i, j \leqslant p-1}\left|\mathfrak{m}_{i j}-1 / p^{2}\right| \leqslant \delta / p^{2}$, or $\mathfrak{m}_{00} \geqslant 1-\delta / p$. Then the claim (4.24) follows.

Thanks to Proposition 3.3, for any $\mathfrak{n}_{i}>0$ and $\varepsilon>0$ sufficiently small,

$$
\log \sum_{j=1}^{p^{d-1}} \prod_{k=0}^{p-1}\left(\frac{\mathfrak{m}_{i k}}{\mathfrak{n}_{i}}\right)^{\frac{d-1}{d} \boldsymbol{w}_{j}(k)} \leqslant-\frac{c(\varepsilon)}{p},
$$


unless $\max _{0 \leqslant k \leqslant p-1}\left|\mathfrak{m}_{i k} / \mathfrak{n}_{i}-1 / p\right| \leqslant \varepsilon / p$, or $\mathfrak{m}_{i 0} / \mathfrak{n}_{i} \geqslant 1-\varepsilon / p$. We decompose $\{0,1, \cdots, p-1\}=I_{1} \cup I_{2} \cup I_{3}$, where

$$
\begin{aligned}
& I_{1}=\left\{0 \leqslant i \leqslant p-1: \max _{0 \leqslant k \leqslant p-1}\left|\mathfrak{m}_{i k} / \mathfrak{n}_{i}-1 / p\right| \leqslant \varepsilon / p\right\}, \\
& I_{2}=\left\{0 \leqslant i \leqslant p-1: \mathfrak{m}_{i 0} / \mathfrak{n}_{i} \geqslant 1-\varepsilon / p\right\}, \quad I_{3}=\{0,1, \cdots, p-1\} \backslash\left(I_{1} \cup I_{2}\right) .
\end{aligned}
$$

Thanks to (4.27), we have

$$
\sum_{i \in I_{3}} \mathfrak{n}_{i} \log \sum_{j=1}^{p^{d-1}} \prod_{k=0}^{p-1}\left(\frac{\mathfrak{m}_{i k}}{\mathfrak{n}_{i}}\right)^{\frac{d-1}{d} \boldsymbol{w}_{j}(k)} \leqslant-\frac{c(\varepsilon)}{p} \sum_{i \in I_{3}} \mathfrak{n}_{i} .
$$

Therefore, if $\sum_{i \in I_{3}} \mathfrak{n}_{i} \geqslant \varepsilon / p$ then (4.26) holds. In the following we assume that $\sum_{i \in I_{3}} \mathfrak{n}_{i} \leqslant \varepsilon / p$. There are several cases:

1. $I_{2}=\emptyset$ : From the discussion above, we have $\sum_{i \in I_{3}} \mathfrak{n}_{i} \leqslant \varepsilon / p$ and $\sum_{i \in I_{1}} \mathfrak{n}_{i} \geqslant 1-\varepsilon / p$. If $I_{3} \neq \emptyset$, we fix any $k \in I_{3}$. By the definition of $I_{1}$, we have $\sum_{i \in I_{1}} \mathfrak{n}_{i} \leqslant \sum_{i \in I_{1}} p \mathfrak{m}_{i k} /(1-\varepsilon) \leqslant p \mathfrak{n}_{k} /(1-\varepsilon) \leqslant \varepsilon /(1-\varepsilon)$, which leads to a contradiction. Therefore $I_{3}=\emptyset$ and $I_{1}=\{0,1,2, \cdots, p-1\}$. Then for any $\mathfrak{m}_{i j}$ and $\mathfrak{m}_{i^{\prime} j^{\prime}}$, we have

$$
\mathfrak{m}_{i j} \leqslant \frac{1+\varepsilon}{1-\varepsilon} \mathfrak{m}_{i i^{\prime}}=\frac{1+\varepsilon}{1-\varepsilon} \mathfrak{m}_{i^{\prime} i} \leqslant \frac{(1+\varepsilon)^{2}}{(1-\varepsilon)^{2}} \mathfrak{m}_{i^{\prime} j^{\prime}}
$$

It follows that $\left|\mathfrak{m}_{i j}-1 / p^{2}\right| \leqslant \delta / p^{2}$ by taking $4 \varepsilon /(1-\varepsilon)^{2} \leqslant \delta$.

2. $I_{2} \neq \emptyset$ : Thanks to (3.27), if $i \in I_{2}$,

$$
\begin{aligned}
& \mathfrak{n}_{i} \log \sum_{j=1}^{p^{d-1}} \prod_{k=0}^{p-1}\left(\frac{\mathfrak{m}_{i k}}{\mathfrak{n}_{i}}\right)^{\frac{d-1}{d} \boldsymbol{w}_{j}(k)} \leqslant-(1+\mathrm{o}(1))(d-1) \mathfrak{n}_{i}\left(1-\frac{\mathfrak{m}_{i 0}}{\mathfrak{n}_{i}}\right) \\
= & -(1+\mathrm{o}(1))(d-1)\left(\mathfrak{n}_{i}-\mathfrak{m}_{i 0}\right)=-(1+\mathrm{o}(1))(d-1) \sum_{1 \leqslant k \leqslant p-1} \mathfrak{m}_{i k} .
\end{aligned}
$$

Therefore, if $\sum_{i \in I_{2}} \sum_{1 \leqslant k \leqslant p-1} \mathfrak{m}_{i k} \geqslant \varepsilon / p^{2}$ then we have that (4.26) holds. In the following, we assume $\sum_{i \in I_{2}} \sum_{1 \leqslant k \leqslant p-1} \mathfrak{m}_{i k} \leqslant \varepsilon / p^{2}$. There are several cases.

(a) $0 \in I_{1}$ : By the definition of $I_{1}$, for any $0 \leqslant k \leqslant p-1$, we have

$$
(1-\varepsilon) \mathfrak{n}_{0} / p \leqslant \mathfrak{m}_{k 0}=\mathfrak{m}_{0 k} \leqslant(1+\varepsilon) \mathfrak{n}_{0} / p .
$$

Fix any $j \in I_{2}$. Again by the definition of $I_{1}, \sum_{i \in I_{1} \backslash\{0\}} \mathfrak{n}_{i} \leqslant \sum_{i \in I_{1} \backslash\{0\}} p \mathfrak{m}_{i j} /(1-\varepsilon / p)=$ $\sum_{i \in I_{1} \backslash\{0\}} p \mathfrak{m}_{j i} /(1-\varepsilon / p) \leqslant \varepsilon /(p-\varepsilon)$. Therefore, $\sum_{1 \leqslant i, k \leqslant p-1} \mathfrak{m}_{i k} \leqslant \sum_{i \in I_{3}} \mathfrak{n}_{i}+\sum_{i \in I_{1} \backslash\{0\}} \mathfrak{n}_{i}+$ $\sum_{i \in I_{2}} \sum_{1 \leqslant k \leqslant p-1} \mathfrak{m}_{i k} \leqslant \varepsilon / p+\varepsilon /(p-\varepsilon)+\varepsilon / p^{2}=\mathrm{O}(\varepsilon) / p$. As a consequence, we get $\mathfrak{m}_{0 k}=\mathfrak{m}_{k 0}=$ $(1+\mathrm{O}(\varepsilon)) /(2 p-1)$, for any $0 \leqslant k \leqslant p-1$, and $\mathfrak{n}_{0}=(1+\mathrm{O}(\varepsilon)) p /(2 p-1)$ and $\mathfrak{n}_{k}=(1+\mathrm{O}(\varepsilon)) /(2 p-1)$ for $1 \leqslant k \leqslant p-1$. In this case, the first term on the lefthand side of (4.26) is small

$$
\frac{d-2}{2} \sum_{i, j=0}^{p-1} \mathfrak{m}_{i j} \ln \frac{\mathfrak{n}_{i} \mathfrak{n}_{j}}{\mathfrak{m}_{i j}} \leqslant(1+\mathrm{O}(\varepsilon)) \frac{d-2}{2}\left(\frac{\ln p}{2 p-1}+\ln \frac{p}{2 p-1}\right)+\mathrm{O}\left(\frac{\varepsilon}{p} \ln \frac{\varepsilon}{p}\right),
$$

and (4.26) holds. 
(b) $0 \in I_{2}$ : By the definition of $I_{2}$, we have $\sum_{i \in I_{2} \backslash\{0\}} \mathfrak{n}_{i} \leqslant \sum_{i \in I_{2} \backslash\{0\}} \mathfrak{m}_{i 0} /(1-\varepsilon / p) \leqslant \sum_{1 \leqslant k \leqslant p-1} \mathfrak{m}_{0 k} /(1-$ $\varepsilon / p) \leqslant \varepsilon / p(p-\varepsilon)$. By the definition of $I_{1}$, we have $\sum_{i \in I_{1}} \mathfrak{n}_{i} \leqslant \sum_{i \in I_{1}} p \mathfrak{m}_{i 0} /(1-\varepsilon) \leqslant \sum_{1 \leqslant k \leqslant p-1} p \mathfrak{m}_{0 k} /(1-$ $\varepsilon) \leqslant \varepsilon / p(1-\varepsilon)$. Combining the discussion above, we get

$$
\begin{aligned}
\mathfrak{m}_{00} & \geqslant \mathfrak{n}_{0}-\varepsilon / p^{2}=1-\sum_{k=1}^{p-1} \mathfrak{n}_{k}-\varepsilon / p^{2} \\
& \geqslant 1-\varepsilon / p(p-\varepsilon)-\varepsilon / p(1-\varepsilon)-\varepsilon / p-\varepsilon / p^{2} .
\end{aligned}
$$

It follows that $\mathfrak{m}_{00} \geqslant 1-\delta / p$ by taking $4 \varepsilon /(1-\varepsilon) \leqslant \delta$.

(c) $0 \in I_{3}$ : By the definition of $I_{1}$, we have $\sum_{i \in I_{1}} \mathfrak{n}_{i} \leqslant \sum_{i \in I_{1}} p \mathfrak{m}_{i 0} /(1-\varepsilon) \leqslant \sum_{1 \leqslant k \leqslant p-1} p \mathfrak{m}_{0 k} /(1-\varepsilon) \leqslant$ $p \mathfrak{n}_{0} /(1-\varepsilon) \leqslant \varepsilon /(1-\varepsilon)$. However, we also know that $\sum_{i \in I_{3}} \mathfrak{n}_{i} \leqslant \varepsilon / p$ and $\sum_{i \in I_{2}} \sum_{1 \leqslant k \leqslant p-1} \mathfrak{m}_{i k} \leqslant$ $\varepsilon / p^{2}$. This contradicts to the fact $\sum_{0 \leqslant i \leqslant p-1} \mathfrak{n}_{i}=1$.

Proof of Proposition 4.2. We further decompose the set of non-equidistributed $p \times p$ symmetric matrices $M=\left[m_{i j}\right]_{0 \leqslant i, j \leqslant p-1}$ into four classes:

1. $p \times p$ symmetric matrices $M=\left[m_{i j}\right]_{0 \leqslant i, j \leqslant p-1} \in \mathcal{N}$ with $\max _{0 \leqslant i, j \leqslant p-1}\left|m_{i j} /(d n)-1 / p^{2}\right| \leqslant \delta / p^{2}$.

2. $p \times p$ symmetric matrices $M=\left[m_{i j}\right]_{0 \leqslant i, j \leqslant p-1} \in \mathcal{N}$ with $\mathfrak{b} p^{3} \ln n / n<\left|m_{00} /(d n)-1\right| \leqslant \delta / p$.

3. $p \times p$ symmetric matrices $M=\left[m_{i j}\right]_{0 \leqslant i, j \leqslant p-1} \in \mathcal{N}$ with $\left|m_{00} /(d n)-1\right| \leqslant \mathfrak{b} p^{3} \ln n / n$.

4. The remaining non-equidistributed $p \times p$ symmetric matrices.

For the first class, $\max _{0 \leqslant i, j \leqslant p-1}\left|m_{i j} /(d n)-1 / p^{2}\right| \leqslant \delta / p^{2}$. The total number of such $p \times p$ symmetric matrices is $e^{\mathrm{O}\left(p^{2} \ln n\right)}$. Given a $p \times p$ symmetric matrix $M=\left[m_{i j}\right]_{0 \leqslant i, j \leqslant p-1}$ in the first class, we will derive a more precise estimate of (4.26), by a perturbation argument. Let

$$
\mathfrak{m}_{i j}=\left(1+\delta_{i j}\right) / p^{2}, \quad i=0,1, \cdots, p-1, \quad j=0,1, \cdots, p-1 .
$$

where $\delta_{i j}=\delta_{j i}$ for $0 \leqslant i<j \leqslant p-1, \sum_{i, j=0}^{p-1} \delta_{i j}=0, \max _{0 \leqslant i, j \leqslant p-1}\left|\delta_{i j}\right| \leqslant \delta$, and $\sum_{i j} \delta_{i j}^{2} \geqslant \mathfrak{b} p^{4} \ln n / n$. We denote,

$$
\mathfrak{n}_{i}=\sum_{j=0}^{p-1} \mathfrak{m}_{i j}=\left(1+\delta_{i}\right) / p, \quad \delta_{i}=\sum_{j=0}^{p-1} \delta_{i j} / p .
$$

We use Taylor expansion, and rewrite the first term in (4.25) as

$$
\begin{aligned}
\frac{d-2}{2} \sum_{i, j=0}^{p-1} \mathfrak{m}_{i j} \ln \frac{\mathfrak{n}_{i} \mathfrak{n}_{j}}{\mathfrak{m}_{i j}} & =(d-2) \sum_{i=0}^{p-1} \mathfrak{n}_{i} \ln \mathfrak{n}_{i}-\frac{d-2}{2} \sum_{i, j=0}^{p-1} \mathfrak{m}_{i j} \ln \mathfrak{m}_{i j} \\
& =(d-2) \sum_{i=0}^{p-1} \frac{1+\delta_{i}}{p} \ln \frac{1+\delta_{i}}{p}-\frac{d-2}{2} \sum_{i, j=0}^{p-1} \frac{1+\delta_{i j}}{p^{2}} \ln \frac{1+\delta_{i j}}{p^{2}} \\
& =(1+\mathrm{O}(\delta))\left(\frac{d-2}{2 p} \sum_{i=0}^{p-1} \delta_{i}^{2}-\frac{d-2}{4 p^{2}} \sum_{i, j=0}^{p-1} \delta_{i j}^{2}\right) .
\end{aligned}
$$


For the second term in (4.25), similar to (3.24) we have

$$
\begin{aligned}
& \log \frac{1}{p^{d-1}} \sum_{j=1}^{p^{d-1}} \prod_{k=0}^{p-1} \mathfrak{m}_{i k}^{\frac{d-1}{d}} \boldsymbol{w}_{j}(k)=\log \frac{1}{p^{d-1}} \sum_{j=1}^{p^{d-1}} \prod_{k=0}^{p-1} e^{\frac{d-1}{d} \boldsymbol{w}_{j}(k) \ln \left(1+\delta_{i k}\right)} \\
= & \left.\log \frac{1}{p^{d-1}} \sum_{j=1}^{p^{d-1}} e^{\frac{d-1}{d} \sum_{k=0}^{p-1} \boldsymbol{w}_{j}(k)\left(\delta_{i k}-\left(1+\mathrm{O}(\delta) \frac{\delta_{i k}^{2}}{2}\right.\right.}\right) \\
= & \log \frac{1}{p^{d-1}} \sum_{j=1}^{p^{d-1}} 1+\frac{d-1}{d} \sum_{k=0}^{p-1} \boldsymbol{w}_{j}(k)\left(\delta_{i k}-\left(1+\mathrm{O}(\delta) \frac{\delta_{i k}^{2}}{2}\right)+(1+\mathrm{O}(\delta)) \frac{(d-1)^{2}}{2 d^{2}}\left(\sum_{k=0}^{p-1} \boldsymbol{w}_{j}(k) \delta_{i k}\right)^{2}\right. \\
= & \left.\log 1+(d-1) \delta_{i}-(1+\mathrm{O}(\delta)) \frac{d-1}{2 p} \sum_{k=0}^{p-1} \delta_{i k}^{2}+\frac{1+\mathrm{O}(\delta)}{p^{d-1}} \frac{(d-1)^{2}}{2 d^{2}} \sum_{j=1}^{p^{d-1}}\left(\sum_{k=0}^{p-1} \boldsymbol{w}_{j}(k) \delta_{i k}\right)^{2}\right)^{2}-\frac{(d-1)^{2}}{2} \delta_{i}^{2} . \\
= & (d-1) \delta_{i}-(1+\mathrm{O}(\delta)) \frac{d-1}{2 p} \sum_{k=0}^{p-1} \delta_{i k}^{2}+\frac{1+\mathrm{O}(\delta)}{p^{d-1}} \frac{(d-1)^{2}}{2 d^{2}} \sum_{j=1}^{p^{d-1}}\left(\sum_{k=0}^{p-1} \boldsymbol{w}_{j}(k) \delta_{i k}\right)^{-\frac{1}{2}}
\end{aligned}
$$

We can rewrite the second term in (4.25) as

$$
\begin{aligned}
& \sum_{i=0}^{p-1} \mathfrak{n}_{i} \log \sum_{j=1}^{p^{d-1}} \prod_{k=0}^{p-1}\left(\frac{\mathfrak{m}_{i k}}{\mathfrak{n}_{i}}\right)^{\frac{d-1}{d} \boldsymbol{w}_{j}(k)}=-(d-1) \sum_{i=0}^{p-1} \mathfrak{n}_{i} \log \mathfrak{n}_{i}+\sum_{i=0}^{p-1} \mathfrak{n}_{i} \log \sum_{j=1}^{p^{d-1}} \prod_{k=0}^{p-1} \mathfrak{m}_{i k}^{\frac{d-1}{d}} \boldsymbol{w}_{j}(k) \\
= & -(d-1) \sum_{i=0}^{p-1} \frac{1+\delta_{i}}{p} \log \frac{1+\delta_{i}}{p}+\sum_{i=0}^{p-1} \frac{1+\delta_{i}}{p} \log \frac{1}{p^{2(d-1)}} \sum_{j=1}^{p^{d-1}} \prod_{k=0}^{p-1} e^{\frac{d-1}{d} \boldsymbol{w}_{j}(k) \ln \left(1+\delta_{i k}\right)} \\
= & (1+\mathrm{O}(\delta))\left(-\frac{d^{2}-3 d+2}{2 p} \sum_{i=0}^{p-1} \delta_{i}^{2}-\frac{d-1}{2 p^{2}} \sum_{i, k=0}^{p-1} \delta_{i k}^{2}+\frac{1}{p^{d}} \frac{(d-1)^{2}}{2 d^{2}} \sum_{i=0}^{p-1} \sum_{j=1}^{p^{d-1}}\left(\sum_{k=0}^{p-1} \boldsymbol{w}_{j}(k) \delta_{i k}\right)^{2}\right) .
\end{aligned}
$$

We get the following estimate of (4.25) by combining (4.30) and (4.31),

$$
\begin{aligned}
& \frac{d-2}{2} \sum_{i, j=0}^{p-1} \mathfrak{m}_{i j} \ln \frac{\mathfrak{n}_{i} \mathfrak{n}_{j}}{\mathfrak{m}_{i j}}+\sum_{i=0}^{p-1} \mathfrak{n}_{i} \log \sum_{j=1}^{p^{d-1}} \prod_{k=0}^{p-1}\left(\frac{\mathfrak{m}_{i k}}{\mathfrak{n}_{i}}\right)^{\frac{d-1}{d} \boldsymbol{w}_{j}(k)} \\
= & (1+\mathrm{O}(\delta))\left(-\frac{(d-2)^{2}}{2 p} \sum_{i=0}^{p-1} \delta_{i}^{2}-\frac{3 d-4}{4 p^{2}} \sum_{i, k=0}^{p-1} \delta_{i k}^{2}+\frac{1}{p^{d}} \frac{(d-1)^{2}}{2 d^{2}} \sum_{i=0}^{p-1} \sum_{j=1}^{p^{d-1}}\left(\sum_{k=0}^{p-1} \boldsymbol{w}_{j}(k) \delta_{i k}\right)^{2}\right) .
\end{aligned}
$$

We denote the $p \times p^{d-1}$ matrix $W=\left[\boldsymbol{w}_{1}, \boldsymbol{w}_{2}, \cdots, \boldsymbol{w}_{p^{d-1}}\right]$ and $p \times p$ matrix $\boldsymbol{\delta}=\left[\delta_{i j}\right]_{0 \leqslant i, j \leqslant p-1}$. Then $\boldsymbol{\delta} \in \operatorname{Sym}_{p}^{0}$, the Hilbert space of $p \times p$ real symmetric matrices with total sum zero, and inner product $\langle A, B\rangle=\operatorname{Tr} A B$. 
We can rewrite (4.32) as a quadratic form on the space $\operatorname{Sym}_{p}^{0}$

$$
\begin{aligned}
& \frac{d-2}{2} \sum_{i, j=0}^{p-1} \mathfrak{m}_{i j} \ln \frac{\mathfrak{n}_{i} \mathfrak{n}_{j}}{\mathfrak{m}_{i j}}+\sum_{i=0}^{p-1} \mathfrak{n}_{i} \log \sum_{j=1}^{p^{d-1}} \prod_{k=0}^{p-1}\left(\frac{\mathfrak{m}_{i k}}{\mathfrak{n}_{i}}\right)^{\frac{d-1}{d} \boldsymbol{w}_{j}(k)} \\
= & (1+\mathrm{O}(\delta))\left(-\frac{(d-2)^{2}}{2 p^{3}}\left\langle\boldsymbol{\delta}, \mathbf{1 1}^{t} \boldsymbol{\delta}\right\rangle-\frac{3 d-4}{4 p^{2}}\langle\boldsymbol{\delta}, \boldsymbol{\delta}\rangle+\frac{1}{p^{d}} \frac{(d-1)^{2}}{2 d^{2}}\left\langle\boldsymbol{\delta}, W W^{t} \boldsymbol{\delta}\right\rangle\right) \\
= & (1+\mathrm{O}(\delta))\left(\frac{d^{2}-d-1}{2 d p^{3}}\left\langle\boldsymbol{\delta}, \mathbf{1 1}^{t} \boldsymbol{\delta}\right\rangle-\frac{d^{2}-2}{4 d p^{2}}\langle\boldsymbol{\delta}, \boldsymbol{\delta}\rangle\right),
\end{aligned}
$$

where we used (2.12),

$$
W W^{t}=d p^{d-2} I_{p}+d(d-1) p^{d-3} \mathbf{1 1}^{t}
$$

We can rewrite the first term in $(4.33)$ as $\left\langle\boldsymbol{\delta}, \mathbf{1 1}^{t} \boldsymbol{\delta}\right\rangle=\langle\boldsymbol{\delta}, \mathcal{L}(\boldsymbol{\delta})\rangle$ where the self-adjoint operator $\mathcal{L}: \mathrm{Sym}_{p}^{0} \mapsto$ $\operatorname{Sym}_{p}^{0}$ is given by $\mathcal{L}(\boldsymbol{\delta})=\mathbf{1 1}^{t} \boldsymbol{\delta} / 2+\boldsymbol{\delta} \mathbf{1 1}^{t} / 2$. The self-adjoint operator $\mathcal{L}$ is diagonalized by

1. If $\boldsymbol{\delta} \in \operatorname{Sym}_{p}^{0}$, with row sums and column sums zero, then $\mathcal{L}(\boldsymbol{\delta})=\mathbf{0}$. The total dimension of such matrices is $p(p-1) / 2$.

2. If $\boldsymbol{\delta}=\left[a_{i}+a_{j}\right]_{0 \leqslant i, j \leqslant p-1}$ for some vector $\boldsymbol{a}=\left(a_{0}, a_{1}, \cdots, a_{p-1}\right) \in \mathbb{R}^{p}$ with $a_{0}+a_{1}+\cdots+a_{p-1}=0$, then $\mathcal{L}(\boldsymbol{\delta})=p \boldsymbol{\delta} / 2$. The total dimension of such matrices is $p-1$.

It follows from the spectral decomposition of the self-adjoint operator $\mathcal{L}$, we get

$$
\left\langle\boldsymbol{\delta}, \mathbf{1 1}^{t} \boldsymbol{\delta}\right\rangle=\langle\boldsymbol{\delta}, \mathcal{L}(\boldsymbol{\delta})\rangle \leqslant p\langle\boldsymbol{\delta}, \boldsymbol{\delta}\rangle / 2,
$$

and

$$
\frac{d-2}{2} \sum_{i, j=0}^{p-1} \mathfrak{m}_{i j} \ln \frac{\mathfrak{n}_{i} \mathfrak{n}_{j}}{\mathfrak{m}_{i j}}+\sum_{i=0}^{p-1} \mathfrak{n}_{i} \log \sum_{j=1}^{p^{d-1}} \prod_{k=0}^{p-1}\left(\frac{\mathfrak{m}_{i k}}{\mathfrak{n}_{i}}\right)^{\frac{d-1}{d} \boldsymbol{w}_{j}(k)} \leqslant-(1+\mathrm{O}(\delta)) \frac{d-1}{4 d p^{2}}\langle\boldsymbol{\delta}, \boldsymbol{\delta}\rangle
$$

The total contribution of terms in (4.20) satisfying $\max _{0 \leqslant i, j \leqslant p-1}\left|m_{i j} /(d n)-1 / p^{2}\right| \leqslant \delta / p^{2}$ is bounded by

$$
\exp \left\{-\left(\frac{\mathfrak{b}(d-1)}{4 d}+\mathrm{o}(1)\right) p^{2} \ln n+\mathrm{O}\left(p^{2} \ln n\right)\right\}=\frac{\mathrm{o}(1)}{n^{(d-2)}},
$$

provided that we take $\mathfrak{b}$ sufficiently large.

For the second class, $\mathfrak{b} p^{3} \ln n / n<\left|m_{00} /(d n)-1\right| \leqslant \delta / p$. The total number of such $p \times p$ symmetric matrices is $e^{\mathrm{O}\left(p^{2} \ln n\right)}$. Given a $p \times p$ symmetric matrix $M=\left[m_{i j}\right]_{0 \leqslant i, j \leqslant p-1}$ in the second class, we will derive a more precise estimate of (4.26), by a perturbative argument. Let $\mathfrak{m}_{00}=1-\delta_{00}$, where $\delta_{00} \leqslant \delta / p$. We recall the decomposition $\{0,1,2, \cdots, p-1\}=I_{1} \cup I_{2} \cup I_{3}$ from (4.28). If $0 \in I_{3}$, then

$$
\mathfrak{n}_{0} \log \sum_{j=1}^{p^{d-1}} \prod_{k=0}^{p-1}\left(\frac{\mathfrak{m}_{0 k}}{\mathfrak{n}_{0}}\right)^{\frac{d-1}{d} \boldsymbol{w}_{j}(k)} \leqslant-\frac{\left(1-\delta_{00}\right) c(\varepsilon)}{p} .
$$


Otherwise, $0 \in I_{2}$. Similar argument as in the proof of Proposition 4.3, we have

$$
\sum_{i \in I_{2} \cup I_{3}} \mathfrak{n}_{i} \log \sum_{j=1}^{p^{d-1}} \prod_{k=0}^{p-1}\left(\frac{\mathfrak{m}_{i k}}{\mathfrak{n}_{i}}\right)^{\frac{d-1}{d} \boldsymbol{w}_{j}(k)} \leqslant-(1+\mathrm{o}(1))(d-1) \sum_{i \in I_{2}} \sum_{1 \leqslant k \leqslant p-1} \mathfrak{m}_{i k}-\frac{c(\varepsilon)}{p} \sum_{i \in I_{3}} \mathfrak{n}_{i} .
$$

Moreover, by the definition of the set $I_{1}$, we have $\sum_{i \in I_{1}} \mathfrak{n}_{i} \leqslant p /(1-\varepsilon) \sum_{i \in I_{1}} \mathfrak{m}_{i 0}=p /(1-\varepsilon) \sum_{i \in I_{1}} \mathfrak{m}_{0 i}$. Therefore,

$$
\begin{aligned}
\delta_{00}=\sum_{\substack{0 \leqslant i, j \leqslant p-1 \\
(i, j) \neq(0,0)}} \mathfrak{m}_{i j} \leqslant \sum_{\substack{i \in I_{2} \\
1 \leqslant k \leqslant p-1}} \mathfrak{m}_{i k}+\sum_{\substack{i \in I_{2} \backslash\{0\} \\
\mathfrak{m}_{i 0}}} \mathfrak{m}_{1-\varepsilon} \sum_{i \in I_{1}} \mathfrak{m}_{0 i}+\sum_{i \in I_{3}} \mathfrak{n}_{i} \\
\leqslant\left(1+\frac{p}{1-\varepsilon}\right) \sum_{\substack{i \in I_{2} \\
1 \leqslant k \leqslant p-1}} \mathfrak{m}_{i k}+\sum_{i \in I_{3}} \mathfrak{n}_{i} .
\end{aligned}
$$

It follows from combining (4.35) and (4.36) we get

$$
\sum_{i \in I_{2} \cup I_{3}} \mathfrak{n}_{i} \log \sum_{j=1}^{p^{d-1}} \prod_{k=0}^{p-1}\left(\frac{\mathfrak{m}_{i k}}{\mathfrak{n}_{i}}\right)^{\frac{d-1}{d} \boldsymbol{w}_{j}(k)} \leqslant-\frac{c(\varepsilon) \delta_{00}}{p} .
$$

Thus, the total contribution of terms in (4.20) satisfying $\mathfrak{b} p^{3} \ln n / n<\left|m_{00} /(d n)-1\right| \leqslant \delta / p$ is bounded by

$$
\exp \left\{-\mathfrak{b} c(\varepsilon) p^{2} \ln n+\mathrm{O}\left(p^{2} \ln n\right)\right\}=\frac{\mathrm{o}(1)}{n^{(d-2)}},
$$

provided that we take $\mathfrak{b}$ sufficiently large.

For the third class, $\left|m_{00} /(d n)-1\right| \leqslant \mathfrak{b} p^{3} \ln n / n$. We denote $\mathcal{M}(r, \ell) \subset \mathcal{M}$ the set of $p \times p$ symmetric matrices $M=\left[m_{i j}\right]_{0 \leqslant i, j \leqslant p-1}$ such that $\ell=n d-m_{00}$, and $r=n-n_{0}(M)$. Then $\sum_{i=1}^{p-1} m_{0 i}=\sum_{i=1}^{p-1} m_{i 0}=$ $\ell-d r$, and $\sum_{1 \leqslant i, j \leqslant p-1} m_{i j}=2 d r-\ell$. Especially, we have $\mathcal{M}(r, \ell)$ is nonempty only if $d r \leqslant \ell \leqslant 2 d r$. The total contribution of terms in (4.20) satisfying $\left|m_{00} /(d n)-1\right| \leqslant \mathfrak{b} p^{3} \ln n / n$ is bounded by

$$
\begin{aligned}
& \frac{2^{n d / 2}(n d / 2) !}{(n d) !} \sum_{\substack{2 \leqslant r \leqslant b p^{3} \ln n / d \\
d r \leqslant \ell \leqslant 2 d r}} \sum_{M \in \mathcal{M}(r, \ell)}\left(\begin{array}{c}
n \\
n_{0}(M), n_{1}(M), \cdots, n_{p-1}(M)
\end{array}\right) \prod_{0 \leqslant i<j \leqslant p-1} m_{i j} ! \prod_{i=0}^{p-1} \frac{m_{i i} !}{2^{m_{i i} / 2}\left(m_{i i} / 2\right) !} \\
& \times \prod_{i=0}^{p-1}\left|\left\{\left(\boldsymbol{u}_{1}, \boldsymbol{u}_{2} \cdots, \boldsymbol{u}_{n_{i}(M)}\right) \in \mathcal{U}_{d, p}^{n_{i}(M)}: \boldsymbol{u}_{1}+\boldsymbol{u}_{2}+\cdots+\boldsymbol{u}_{n_{i}(M)}=\left(m_{i 0}, m_{i 1}, \cdots, m_{i p-1}\right)\right\}\right| .
\end{aligned}
$$

We reestimate the first factor on the righthand side of (4.38),

$$
\begin{aligned}
& \frac{2^{n d / 2}(n d / 2) !}{(n d) !}\left(\begin{array}{c}
n \\
n_{0}(M), n_{1}(M), \cdots, n_{p-1}(M)
\end{array}\right) \prod_{0 \leqslant i<j \leqslant p-1} m_{i j} ! \prod_{i=0}^{p-1} \frac{m_{i i} !}{2^{m_{i i} / 2}\left(m_{i i} / 2\right) !} \\
\leqslant & \frac{e^{\mathrm{O}(r)}}{n^{\ell / 2-r}} \frac{1}{n_{1}(M) ! n_{2}(M) ! \cdots n_{p-1}(M) !} \prod_{0 \leqslant i<j \leqslant p-1} m_{i j} ! \prod_{i=1}^{p-1} \frac{m_{i i} !}{2^{m_{i i} / 2}\left(m_{i i} / 2\right) !} .
\end{aligned}
$$


For the number of walk paths in (4.38), we notice that $\boldsymbol{w}_{j}(1)+\boldsymbol{w}_{j}(2)+\cdots \boldsymbol{w}_{j}(p-1) \geqslant 2$ for $2 \leqslant j \leqslant p^{d-1}$. Moreover, we have $\boldsymbol{u}_{1}+\boldsymbol{u}_{2}+\cdots+\boldsymbol{u}_{n_{0}(M)}=\left(m_{00}, m_{01}, \cdots, m_{0 p-1}\right)$, with $m_{01}+m_{02}+\cdots+m_{0 p-1}=$ $d n_{0}(M)-m_{00}=\ell-d r$. Therefore $\boldsymbol{u}_{i}=\boldsymbol{w}_{1}$ for all $1 \leqslant i \leqslant n_{0}(M)$, except for at most $(\ell-d r) / 2$ of them. Therefore, we have

$$
\begin{aligned}
& \left|\left\{\left(\boldsymbol{u}_{1}, \boldsymbol{u}_{2} \cdots, \boldsymbol{u}_{n_{0}(M)}\right) \in \mathcal{U}_{d, p}^{n_{0}(M)}: \boldsymbol{u}_{1}+\boldsymbol{u}_{2}+\cdots+\boldsymbol{u}_{n_{0}(M)}=\left(m_{00}, m_{01}, \cdots, m_{0 p-1}\right)\right\}\right| \\
\leqslant & \frac{(\ell-d r) !}{m_{01} ! m_{02} ! \cdots m_{0 p-1} !} \sum_{k=1}^{(\ell-d r) / 2} n^{k}\left(\begin{array}{c}
d m-k-1 \\
k-1
\end{array}\right) \leqslant \mathrm{O}(1) \frac{(\ell-d r) !}{m_{01} ! m_{02} ! \cdots m_{0 p-1} !} n^{(\ell-d r) / 2} .
\end{aligned}
$$

For the number of walk paths in (4.38) corresponding to $i=1,2, \cdots, p-1$, we have the trivial bound

$$
\begin{aligned}
& \left|\left\{\left(\boldsymbol{u}_{1}, \boldsymbol{u}_{2} \cdots, \boldsymbol{u}_{n_{i}(M)}\right) \in \mathcal{U}_{d, p}^{n_{i}(M)}: \boldsymbol{u}_{1}+\boldsymbol{u}_{2}+\cdots+\boldsymbol{u}_{n_{i}(M)}=\left(m_{i 0}, m_{i 1}, \cdots, m_{i p-1}\right)\right\}\right| \\
\leqslant & \frac{\left(d n_{i}(M)\right) !}{m_{i 0} ! m_{i 1} ! \cdots m_{i p-1} !} .
\end{aligned}
$$

Combining the estimates (4.40) and (4.41), we get the following bound on the number of walk paths in $(4.38)$,

$$
\begin{aligned}
& \prod_{i=0}^{p-1}\left|\left\{\left(\boldsymbol{u}_{1}, \boldsymbol{u}_{2} \cdots, \boldsymbol{u}_{n_{i}(M)}\right) \in \mathcal{U}_{d, p}^{n_{i}(M)}: \boldsymbol{u}_{1}+\boldsymbol{u}_{2}+\cdots+\boldsymbol{u}_{n_{i}(M)}=\left(m_{i 0}, m_{i 1}, \cdots, m_{i p-1}\right)\right\}\right| \\
\leqslant & \mathrm{O}(1) n^{(\ell-d r) / 2} \frac{(\ell-d k) !}{m_{01} ! m_{02} ! \cdots m_{0 p-1} !} \prod_{i=1}^{p-1} \frac{\left(d n_{i}(M)\right) !}{m_{i 0} ! m_{i 1} ! \cdots m_{i p-1} !} .
\end{aligned}
$$

The total contribution of terms in (4.20) satisfying $\left|m_{00} /(d n)-1\right| \leqslant \mathfrak{b} p^{3} \ln n / n$ is bounded by

$$
\begin{aligned}
& \sum_{\substack{2 \leqslant r \leqslant \mathfrak{b} p^{3} \ln n / d \\
d r \leqslant \ell \leqslant 2 d r}} \sum_{M \in \mathcal{M}(r, \ell)} \frac{e^{\mathrm{O}(r)}(\ell-d r) !}{n^{(d / 2-1) r}} \prod_{i=1}^{p-1} \frac{\left(d n_{i}(M)\right) !}{n_{i}(M) !} \prod_{0 \leqslant i<j \leqslant p-1} \frac{1}{m_{i j} !} \prod_{i=1}^{p-1} \frac{1}{2^{m_{i i} / 2}\left(m_{i i} / 2\right) !} \\
\leqslant & \sum_{\substack{2 \leqslant r \leqslant \mathfrak{b} p^{3} \ln n / d \\
d r \leqslant \ell \leqslant 2 d r}} \frac{e^{\mathrm{O}(r)}(\ell-d r) ! r^{(d-1) r}}{n^{(d / 2-1) r}} \sum_{M \in \mathcal{M}(r, \ell)} \prod_{i=1}^{p-1} \frac{1}{m_{i 0} !} \prod_{1 \leqslant i \leqslant j \leqslant p-1} \frac{1}{\left(m_{i j} /\left(1+\delta_{i j}\right)\right) !} \\
\leqslant & \sum_{\substack{2 \leqslant r \leqslant \mathfrak{b} p^{3} \ln n / d \\
d r \leqslant \ell \leqslant 2 d r}} \frac{e^{\mathrm{O}(r)} r^{(d-1) r}}{n^{(d / 2-1) r}} \frac{p^{d r}}{(d r-\ell / 2) !} \\
\leqslant & \sum_{2 \leqslant r \leqslant \mathfrak{b} p^{3} \ln n / d}\left(\frac{\mathrm{O}(1) p^{d} r^{(d / 2-1)}}{n^{(d / 2-1)}}\right)^{r} \leqslant \frac{\mathrm{O}(1) p^{2 d}}{n^{d-2}},
\end{aligned}
$$

provided that $p \ll n^{(d-2) /(5 d-6)}$.

For the last class, the total number of such $p \times p$ symmetric matrices is $e^{\mathrm{O}\left(p^{2} \ln n\right)}$, and thanks to Proposition 4.3, each term is exponentially small, i.e. $e^{-c(\delta) n / p^{2}}$. Therefore the total contribution is

$$
\exp \left\{-c(\delta) n / p^{2}+\mathrm{O}\left(p^{2} \ln n\right)\right\}=\frac{\mathrm{o}(1)}{n^{(d-2)}} .
$$


(4.44).

The claim (4.20) follows from combining the discussion of all four cases, (4.34), (4.37), (4.43) and

\section{References}

[1] A. Basak, N. Cook, and O. Zeitouni. Circular law for the sum of random permutation matrices. Electron. J. Probab., 23:51 pp., 2018.

[2] A. Basak and M. Rudelson. The circular law for sparse non-hermitian matrices. preprint: arXiv: 1707.03675, 2017.

[3] A. Basak and M. Rudelson. Invertibility of sparse non-Hermitian matrices. Adv. Math., 310:426-483, 2017.

[4] E. A. Bender and E. R. Canfield. The asymptotic number of labeled graphs with given degree sequences. J. Combinatorial Theory Ser. A, 24(3):296-307, 1978.

[5] B. Bollobás. A probabilistic proof of an asymptotic formula for the number of labelled regular graphs. European J. Combin., 1(4):311-316, 1980.

[6] J. Bourgain, V. H. Vu, and P. M. Wood. On the singularity probability of discrete random matrices. $J$. Funct. Anal., 258(2):559-603, 2010.

[7] N. A. Cook. The circular law for random regular digraphs. preprint, arXiv: 1703.05839, 2017.

[8] N. A. Cook. On the singularity of adjacency matrices for random regular digraphs. Probab. Theory Related Fields, 167(1-2):143-200, 2017.

[9] K. P. Costello, T. Tao, and V. Vu. Random symmetric matrices are almost surely nonsingular. Duke Math. J., 135(2):395-413, 2006.

[10] K. P. Costello and V. H. Vu. The rank of random graphs. Random Structures Algorithms, 33(3):269-285, 2008.

[11] A. Ferber, K. Luh, G. McKinley, and W. Samotij. Some problems on random discrete matrices. In preparation, 2018.

[12] A. Frieze. Random structures and algorithms. In Proceedings of the International Congress of Mathematicians-Seoul 2014. Vol. 1, pages 311-340. Kyung Moon Sa, Seoul, 2014.

[13] J. Kahn, J. Komlós, and E. Szemerédi. On the probability that a random \pm 1 -matrix is singular. $J$. Amer. Math. Soc., 8(1):223-240, 1995.

[14] J. Komlós. On the determinant of (0, 1) matrices. Studia Sci. Math. Hungar, 2:7-21, 1967.

[15] J. Komlós. On the determinant of random matrices. Studia Sci. Math. Hungar., 3:387-399, 1968.

[16] B. Landon, P. Sosoe, and H.-T. Yau. Fixed energy universality of Dyson Brownian motion. preprint, arXiv: 1609.09011, 2016.

[17] A. E. Litvak, A. Lytova, K. Tikhomirov, N. Tomczak-Jaegermann, and P. Youssef. Adjacency matrices of random digraphs: singularity and anti-concentration. J. Math. Anal. Appl., 445(2):1447-1491, 2017. 
[18] A. E. Litvak, A. Lytova, K. Tikhomirov, N. Tomczak-Jaegermann, and P. Youssef. The smallest singular value of a shifted d-regular random square. preprint, arXiv:170\%.02635, 2017.

[19] A. E. Litvak, A. Lytova, K. Tikhomirov, N. Tomczak-Jaegermann, and P. Youssef. The rank of random regular digraphs of constant degree. preprint, arXiv: 1801.05577, 2018.

[20] A. E. Litvak, A. Lytova, K. Tikhomirov, N. Tomczak-Jaegermann, and P. Youssef. Structure of eigenvectors of random regular digraphs. preprint, arXiv:1801.05575, 2018.

[21] A. Mészáros. The distribution of sandpile groups of random regular graphs. preprint, arXiv: 1806.03736, 2018.

[22] H. H. Nguyen. Inverse Littlewood-Offord problems and the singularity of random symmetric matrices. Duke Math. J., 161(4):545-586, 2012.

[23] H. H. Nguyen and W. M. Matchett. Cokernels of adjacency matrices of random r-regular graphs. preprint, arXiv: 1806.10068, 2018.

[24] M. Rudelson and R. Vershynin. The Littlewood-Offord problem and invertibility of random matrices. Adv. Math., 218(2):600-633, 2008.

[25] M. Rudelson and R. Vershynin. Smallest singular value of a random rectangular matrix. Comm. Pure Appl. Math., 62(12):1707-1739, 2009.

[26] T. Tao and V. Vu. On the singularity probability of random Bernoulli matrices. J. Amer. Math. Soc., 20(3):603-628, 2007.

[27] R. Vershynin. Invertibility of symmetric random matrices. Random Structures Algorithms, 44(2):135$182,2014$.

[28] V. Vu. Random discrete matrices. In Horizons of combinatorics, volume 17 of Bolyai Soc. Math. Stud., pages 257-280. Springer, Berlin, 2008.

[29] V. H. Vu. Combinatorial problems in random matrix theory. In Proceedings of the International Congress of Mathematicians -Seoul 2014. Vol. IV, pages 489-508. Kyung Moon Sa, Seoul, 2014.

[30] N. Wormald. Enumeration of labelled graphs. I. 3-connected graphs. J. London Math. Soc. (2), 19(1):7$12,1979$.

[31] N. C. Wormald. Enumeration of labelled graphs. II. Cubic graphs with a given connectivity. J. London Math. Soc. (2), 20(1):1-7, 1979. 